\title{
Convex Minimization with Constraints of Systems of Variational Inequalities, Mixed Equilibrium, Variational Inequality, and Fixed Point Problems
}

\author{
Lu-Chuan Ceng, ${ }^{1}$ Cheng-Wen Liao, ${ }^{2}$ Chin-Tzong Pang, ${ }^{3}$ and Ching-Feng Wen ${ }^{4}$ \\ ${ }^{1}$ Department of Mathematics, Shanghai Normal University, and Scientific Computing Key Laboratory of Shanghai Universities, \\ Shanghai 200234, China \\ ${ }^{2}$ Department of Food and Beverage Management, Vanung University, Chung-Li 320061, Taiwan \\ ${ }^{3}$ Department of Information Management and Innovation Center for Big Data and Digital Convergence, Yuan Ze University, \\ Chung-Li 32003, Taiwan \\ ${ }^{4}$ Center for Fundamental Science, Kaohsiung Medical University, Kaohsiung 807, Taiwan
}

Correspondence should be addressed to Chin-Tzong Pang; imctpang@saturn.yzu.edu.tw

Received 17 March 2014; Accepted 26 March 2014; Published 8 May 2014

Academic Editor: Jen-Chih Yao

Copyright @ 2014 Lu-Chuan Ceng et al. This is an open access article distributed under the Creative Commons Attribution License, which permits unrestricted use, distribution, and reproduction in any medium, provided the original work is properly cited.

We introduce and analyze one iterative algorithm by hybrid shrinking projection method for finding a solution of the minimization problem for a convex and continuously Fréchet differentiable functional, with constraints of several problems: finitely many generalized mixed equilibrium problems, finitely many variational inequalities, the general system of variational inequalities and the fixed point problem of an asymptotically strict pseudocontractive mapping in the intermediate sense in a real Hilbert space. We prove strong convergence theorem for the iterative algorithm under suitable conditions. On the other hand, we also propose another iterative algorithm by hybrid shrinking projection method for finding a fixed point of infinitely many nonexpansive mappings with the same constraints, and derive its strong convergence under mild assumptions.

\section{Introduction}

Let $C$ be a nonempty closed convex subset of a real Hilbert space $H$ and let $P_{C}$ be the metric projection of $H$ onto $C$. Let $S: C \rightarrow H$ be a nonlinear mapping on $C$. We denote by $\operatorname{Fix}(S)$ the set of fixed points of $S$ and by $\mathbf{R}$ the set of all real numbers. A mapping $S: C \rightarrow H$ is called $L$-Lipschitz continuous if there exists a constant $L \geq 0$ such that

$$
\|S x-S y\| \leq L\|x-y\|, \quad \forall x, y \in C .
$$

In particular, if $L=1$ then $S$ is called a nonexpansive mapping; if $L \in[0,1)$ then $S$ is called a contraction. A mapping $V$ is called strongly positive on $H$ if there exists a constant $\bar{\gamma}>0$ such that

$$
\langle V x, x\rangle \geq \bar{\gamma}\|x\|^{2}, \quad \forall x \in H .
$$

Let $A: C \rightarrow H$ be a nonlinear mapping on $C$. We consider the following variational inequality problem (VIP): find a point $x \in C$ such that

$$
\langle A x, y-x\rangle \geq 0, \quad \forall y \in C .
$$

The solution set of VIP (3) is denoted by $\operatorname{VI}(C, A)$.

Let $\varphi: C \rightarrow \mathbf{R}$ be a real-valued function, let $A$ : $H \rightarrow H$ be a nonlinear mapping, and let $\Theta: C \times C \rightarrow \mathbf{R}$ be a bifunction. Peng and Yao [1] introduced the following generalized mixed equilibrium problem (GMEP) of finding $x \in C$ such that

$$
\Theta(x, y)+\varphi(y)-\varphi(x)+\langle A x, y-x\rangle \geq 0, \quad \forall y \in C .
$$

We denote the set of solutions of GMEP (4) by $\operatorname{GMEP}(\Theta, \varphi, A)$. The GMEP (4) is very general in the sense that it includes, as special cases, optimization problems, 
variational inequalities, minimax problems, and Nash equilibrium problems in noncooperative games. It covers problems considered in [2-5].

It is assumed as in [1] that $\Theta: C \times C \rightarrow \mathbf{R}$ is a bifunction satisfying conditions (A1)-(A4) and $\varphi: C \rightarrow \mathbf{R}$ is a lower semicontinuous and convex function with restriction (B1) or (B2), where

(A1) $\Theta(x, x)=0$ for all $x \in C$;

(A2) $\Theta$ is monotone; that is, $\Theta(x, y)+\Theta(y, x) \leq 0$ for any $x, y \in C$;

(A3) $\Theta$ is upper-hemicontinuous; that is, for each $x, y, z \in$ C,

$$
\limsup _{t \rightarrow 0^{+}} \Theta(t z+(1-t) x, y) \leq \Theta(x, y) ;
$$

(A4) $\Theta(x, \cdot)$ is convex and lower semicontinuous for each $x \in C$;

(B1) for each $x \in H$ and $r>0$, there exists a bounded subset $D_{x} \subset C$ and $y_{x} \in C$ such that, for any $z \in$ $C \backslash D_{x}$,

$$
\Theta\left(z, y_{x}\right)+\varphi\left(y_{x}\right)-\varphi(z)+\frac{1}{r}\left\langle y_{x}-z, z-x\right\rangle<0
$$

(B2) $C$ is a bounded set.

Given a positive number $r>0$, let $T_{r}^{(\Theta, \varphi)}: H \rightarrow C$ be the solution set of the auxiliary mixed equilibrium problem; that is, for each $x \in H$,

$$
\begin{aligned}
& T_{r}^{(\Theta, \varphi)}(x):=\{y \in C: \Theta(y, z)+\varphi(z)-\varphi(y) \\
&\left.+\frac{1}{r}\langle y-x, z-y\rangle \geq 0, \forall z \in C\right\} .
\end{aligned}
$$

Let $F_{1}, F_{2}: C \rightarrow H$ be two mappings. Consider the following general system of variational inequalities (GSVI) [6] of finding $\left(x^{*}, y^{*}\right) \in C \times C$ such that

$$
\begin{array}{ll}
\left\langle\nu_{1} F_{1} y^{*}+x^{*}-y^{*}, x-x^{*}\right\rangle \geq 0, & \forall x \in C, \\
\left\langle\nu_{2} F_{2} x^{*}+y^{*}-x^{*}, x-y^{*}\right\rangle \geq 0, & \forall x \in C,
\end{array}
$$

where $\nu_{1}>0$ and $\nu_{2}>0$ are two constants. In 2008, Ceng et al. [6] transformed the GSVI (8) into a fixed point problem in the following way.

Proposition CWY (see [6]). For given $\bar{x}, \bar{y} \in C,(\bar{x}, \bar{y})$ is a solution of the GSVI (8) if and only if $\bar{x}$ is a fixed point of the mapping $G: C \rightarrow C$ defined by

$$
G x=P_{C}\left(I-v_{1} F_{1}\right) P_{C}\left(I-v_{2} F_{2}\right) x, \quad \forall x \in C,
$$

where $\bar{y}=P_{C}\left(I-v_{2} F_{2}\right) \bar{x}$.

In particular, if the mapping $F_{j}: C \rightarrow H$ is $\zeta_{j}$-inversestrongly monotone for $j=1,2$, then the mapping $G$ is nonexpansive provided $\nu_{j} \in\left(0,2 \zeta_{j}\right]$ for $j=1,2$. We denote by $\operatorname{GSVI}(G)$ the fixed point set of the mapping $G$.
Let $\lambda_{n, 1}, \lambda_{n, 2}, \ldots, \lambda_{n, N} \in(0,1], n \geq 1$. Given the nonexpansive self-mappings $T_{1}, T_{2}, \ldots, T_{N}$ on $C$, for each $n \geq$ 1 , the mappings $U_{n, 1}, U_{n, 2}, \ldots, U_{n, N}$ are defined by

$$
\begin{gathered}
U_{n, 1}=\lambda_{n, 1} T_{1}+\left(1-\lambda_{n, 1}\right) I, \\
U_{n, 2}=\lambda_{n, 2} T_{n} U_{n, 1}+\left(1-\lambda_{n, 2}\right) I, \\
U_{n, n-1}=\lambda_{n-1} T_{n-1} U_{n, n}+\left(1-\lambda_{n-1}\right) I, \\
\vdots \\
U_{n, N-1}=\lambda_{n, N-1} T_{N-1} U_{n, N-2}+\left(1-\lambda_{n, N-1}\right) I, \\
W_{n}:=U_{n, N}=\lambda_{n, N} T_{N} U_{n, N-1}+\left(1-\lambda_{n, N}\right) I .
\end{gathered}
$$

The $W_{n}$ is called the $W$-mapping generated by $T_{1}, \ldots, T_{N}$ and $\lambda_{n, 1}, \lambda_{n, 2}, \ldots, \lambda_{n, N}$. Note that the nonexpansivity of $T_{i}$ implies the one of $W_{n}$. In 2012, combining the hybrid steepest-descent method in [7] and viscosity approximation method, Ceng et al. [8] proposed and analyzed the following hybrid iterative algorithm for finding a common element of the solution set of GMEP (4) and the fixed point set of finitely many nonexpansive mappings $\left\{T_{i}\right\}_{i=1}^{N}$.

Theorem CGY (see [8, Theorem 3.1]). Let $C$ be a nonempty closed convex subset of a real Hilbert space $H$. Let $\Theta: C \times$ $C \rightarrow \mathbf{R}$ be a bifunction satisfying assumptions (A1)-(A4) and let $\varphi: C \rightarrow \mathbf{R}$ be a lower semicontinuous and convex function with restriction (B1) or (B2). Let the mapping $A: H \rightarrow H$ be $\delta$-inverse-strongly monotone, and let $\left\{T_{i}\right\}_{i=1}^{N}$ be a finite family of nonexpansive mappings on $H$ such that $\Omega:=\cap_{i=1}^{N} F i x\left(T_{i}\right) \cap$ $\operatorname{GMEP}(\Theta, \varphi, A) \neq \emptyset$. Let $F: H \rightarrow H$ be a $\kappa$-Lipschitzian and $\eta$-strongly monotone operator with positive constants $\kappa, \eta>0$ and $Q: H \rightarrow H$ an l-Lipschitzian mapping with constant $l \geq 0$. Let $0<\mu<2 \eta / \kappa^{2}$ and $0 \leq \gamma l<\tau$, where $\tau=1-$ $\sqrt{1-\mu\left(2 \eta-\mu \kappa^{2}\right)}$. Suppose $\left\{\alpha_{n}\right\}$ and $\left\{\beta_{n}\right\}$ are two sequences in $(0,1),\left\{\gamma_{n}\right\}$ is a sequence in $(0,2 \delta]$, and $\left\{\lambda_{n, i}\right\}_{i=1}^{N}$ is a sequence in $[a, b]$ with $0<a \leq b<1$. For every $n \geq 1$, let $W_{n}$ be the $W$-mapping generated by $T_{1}, \ldots, T_{N}$ and $\lambda_{n, 1}, \lambda_{n, 2}, \ldots, \lambda_{n, N}$. Given $x_{1} \in H$ arbitrarily, suppose the sequences $\left\{x_{n}\right\}$ and $\left\{u_{n}\right\}$ are generated iteratively by

$$
\begin{array}{r}
\Theta\left(u_{n}, y\right)+\varphi(y)-\varphi\left(u_{n}\right)+\left\langle A x_{n}, y-u_{n}\right\rangle \\
+\frac{1}{r_{n}}\left\langle y-u_{n}, u_{n}-x_{n}\right\rangle \geq 0, \quad \forall y \in C, \\
x_{n+1}=\alpha_{n} \gamma Q x_{n}+\beta_{n} x_{n}+\left(\left(1-\beta_{n}\right) I-\alpha_{n} \mu F\right) W_{n} u_{n}, \\
\forall n \geq 1,
\end{array}
$$

where the sequences $\left\{\alpha_{n}\right\},\left\{\beta_{n}\right\}$, and $\left\{r_{n}\right\}$ and the finite family of sequences $\left\{\lambda_{n, i}\right\}_{i=1}^{N}$ satisfy the following conditions:

(i) $\lim _{n \rightarrow \infty} \alpha_{n}=0$ and $\sum_{n=1}^{\infty} \alpha_{n}=\infty$;

(ii) $0<\liminf _{n \rightarrow \infty} \beta_{n} \leq \limsup _{n \rightarrow \infty} \beta_{n}<1$;

(iii) $0<\liminf _{n \rightarrow \infty} r_{n} \leq \limsup _{n \rightarrow \infty} r_{n}<2 \delta$ and $\lim _{n \rightarrow \infty}\left(r_{n+1}-r_{n}\right)=0$;

(iv) $\lim _{n \rightarrow \infty}\left(\lambda_{n+1, i}-\lambda_{n, i}\right)=0$ for $i=1,2, \ldots, N$. 
Then both $\left\{x_{n}\right\}$ and $\left\{u_{n}\right\}$ converge strongly to $x^{*}=P_{\Omega}(I-\mu F+$ $\gamma Q) x^{*}$, which is the unique solution in $\Omega$ to the VIP

$$
\left\langle(\mu F-\gamma Q) x^{*}, x^{*}-x\right\rangle \leq 0, \quad \forall x \in \Omega .
$$

Let $f: C \rightarrow \mathbf{R}$ be a convex and continuously Fréchet differentiable functional. Consider the convex minimization problem (CMP) of minimizing $f$ over the constraint set $C$

$$
\text { minimize }\{f(x): x \in C\} \text {. }
$$

We denote by $\Gamma$ the set of minimizers of CMP (13).

Next, recall some concepts. Let $C$ be a nonempty subset of a normed space $X$. A mapping $S: C \rightarrow C$ is called uniformly Lipschitzian if there exists a constant $\mathscr{L}>0$ such that

$$
\left\|S^{n} x-S^{n} y\right\| \leq \mathscr{L}\|x-y\|, \quad \forall n \geq 1, \forall x, y \in C .
$$

Recently, Kim and Xu [9] introduced the concept of asymptotically $k$-strict pseudocontractive mappings in a Hilbert space as below.

Definition 1. Let $C$ be a nonempty subset of a Hilbert space $H$. A mapping $S: C \rightarrow C$ is said to be an asymptotically $k$ strict pseudocontractive mapping with sequence $\left\{\gamma_{n}\right\}$ if there exists a constant $k \in[0,1)$ and a sequence $\left\{\gamma_{n}\right\}$ in $[0, \infty)$ with $\lim _{n \rightarrow \infty} \gamma_{n}=0$ such that

$$
\begin{array}{r}
\left\|S^{n} x-S^{n} y\right\|^{2} \leq\left(1+\gamma_{n}\right)\|x-y\|^{2} \\
+k\left\|x-S^{n} x-\left(y-S^{n} y\right)\right\|^{2}, \\
\forall n \geq 1, \quad \forall x, y \in C .
\end{array}
$$

It is important to note that every asymptotically $k$ strict pseudocontractive mapping with sequence $\left\{\gamma_{n}\right\}$ is a uniformly $\mathscr{L}$-Lipschitzian mapping with $\mathscr{L}=\sup \{(k+$ $\left.\left.\sqrt{1+(1-k) \gamma_{n}}\right) /(1+k): n \geq 1\right\}$. Subsequently, Sahu et al. [10] considered the concept of asymptotically $k$-strict pseudocontractive mappings in the intermediate sense, which are not necessarily Lipschitzian.

Definition 2. Let $C$ be a nonempty subset of a Hilbert space $H$. A mapping $S: C \rightarrow C$ is said to be an asymptotically $k$ strict pseudocontractive mapping in the intermediate sense with sequence $\left\{\gamma_{n}\right\}$ if there exist a constant $k \in[0,1)$ and a sequence $\left\{\gamma_{n}\right\}$ in $[0, \infty)$ with $\lim _{n \rightarrow \infty} \gamma_{n}=0$ such that

$$
\begin{array}{r}
\limsup _{n \rightarrow \infty} \sup _{x, y \in C}\left(\left\|S^{n} x-S^{n} y\right\|^{2}-\left(1+\gamma_{n}\right)\|x-y\|^{2}\right. \\
\left.-k\left\|x-S^{n} x-\left(y-S^{n} y\right)\right\|^{2}\right) \leq 0 .
\end{array}
$$

Put $c_{n}:=\max \left\{0, \sup _{x, y \in C}\left(\left\|S^{n} x-S^{n} y\right\|^{2}-\left(1+\gamma_{n}\right)\|x-y\|^{2}-\right.\right.$ $\left.\left.k\left\|x-S^{n} x-\left(y-S^{n} y\right)\right\|^{2}\right)\right\}$. Then $c_{n} \geq 0(\forall n \geq 1), c_{n} \rightarrow$ $0(n \rightarrow \infty)$, and there holds the relation

$$
\begin{aligned}
& \left\|S^{n} x-S^{n} y\right\|^{2} \\
& \leq\left(1+\gamma_{n}\right)\|x-y\|^{2}+k\left\|x-S^{n} x-\left(y-S^{n} y\right)\right\|^{2}+c_{n}, \\
& \forall n \geq 1, \quad \forall x, y \in C .
\end{aligned}
$$

In 2009, Sahu et al. [10] first established one weak convergence theorem for the following Mann-type iterative scheme:

$$
\begin{aligned}
x_{1} & =x \in C \quad \text { chosen arbitrary, } \\
x_{n+1} & =\left(1-\alpha_{n}\right) x_{n}+\alpha_{n} S^{n} x_{n}, \quad \forall n \geq 1,
\end{aligned}
$$

where $0<\delta \leq \alpha_{n} \leq 1-k-\delta, \quad \sum_{n=1}^{\infty} \alpha_{n} c_{n}<\infty$, and $\sum_{n=1}^{\infty} \gamma_{n}<$ $\infty$, and then obtained another strong convergence theorem for the following hybrid CQ iterative scheme:

$$
\begin{aligned}
x_{1} & =x \in C \quad \text { chosen arbitrary, } \\
y_{n} & =\left(1-\alpha_{n}\right) x_{n}+\alpha_{n} S^{n} x_{n}, \\
C_{n} & =\left\{z \in C:\left\|y_{n}-z\right\|^{2} \leq\left\|x_{n}-z\right\|^{2}+\theta_{n}\right\}, \\
Q_{n} & =\left\{z \in C:\left\langle x_{n}-z, x-x_{n}\right\rangle \geq 0\right\}, \\
x_{n+1} & =P_{C_{n} \cap Q_{n}} x, \quad \forall n \geq 1,
\end{aligned}
$$

where $0<\delta \leq \alpha_{n} \leq 1-k, \theta_{n}=c_{n}+\gamma_{n} \Delta_{n}$, and $\Delta_{n}=\sup \left\{\left\|x_{n}-z\right\|^{2}: z \in \operatorname{Fix}(S)\right\}<\infty$. Subsequently, the above iterative schemes are extended to develop new iterative algorithms for finding a common solution of the VIP and the fixed point problem of an asymptotically strict pseudocontractive mapping in the intermediate sense; see, for example, [11-13].

Motivated and inspired by the above facts, we first introduce and analyze one iterative algorithm by hybrid shrinking projection method for finding a solution of the CMP (13) with constraints of several problems: finitely many GMEPs, finitely many VIPs, the GSVI (8), and the fixed point problem of an asymptotically strict pseudocontractive mapping in the intermediate sense in a real Hilbert space. We prove strong convergence theorem for the iterative algorithm under suitable conditions. The iterative algorithm is based on shrinking projection method, Korpelevich's extragradient method, hybrid steepest-descent method in [7], viscosity approximation method, averaged mapping approach to the GPA in [14], and strongly positive bounded linear operator technique. On the other hand, we also propose another iterative algorithm by hybrid shrinking projection method for finding a fixed point of infinitely many nonexpansive mappings with the same constraints. We derive its strong convergence under mild assumptions. The results obtained in this paper improve and extend the corresponding results announced by many others.

\section{Preliminaries}

Throughout this paper, we assume that $H$ is a real Hilbert space whose inner product and norm are denoted by $\langle\cdot, \cdot\rangle$ and $\|\cdot\|$, respectively. Let $C$ be a nonempty closed convex subset of $H$. We write $x_{n} \rightarrow x$ to indicate that the sequence $\left\{x_{n}\right\}$ converges weakly to $x$ and $x_{n} \rightarrow x$ to indicate that the sequence $\left\{x_{n}\right\}$ converges strongly to $x$. Moreover, we use 
$\omega_{w}\left(x_{n}\right)$ to denote the weak $\omega$-limit set of the sequence $\left\{x_{n}\right\}$; that is,

$$
\begin{aligned}
\omega_{w}\left(x_{n}\right):= & \left\{x \in H: x_{n_{i}} \rightarrow x\right. \text { for some subsequence } \\
& \left.\left\{x_{n_{i}}\right\} \text { of }\left\{x_{n}\right\}\right\} .
\end{aligned}
$$

Recall that a mapping $A: C \rightarrow H$ is called

(i) monotone if

$$
\langle A x-A y, x-y\rangle \geq 0, \quad \forall x, y \in C ;
$$

(ii) $\eta$-strongly monotone if there exists a constant $\eta>0$ such that

$$
\langle A x-A y, x-y\rangle \geq \eta\|x-y\|^{2}, \quad \forall x, y \in C ;
$$

(iii) $\alpha$-inverse-strongly monotone if there exists a constant $\alpha>0$ such that

$$
\langle A x-A y, x-y\rangle \geq \alpha\|A x-A y\|^{2}, \quad \forall x, y \in C .
$$

It is obvious that if $A$ is $\alpha$-inverse-strongly monotone, then $A$ is monotone and $1 / \alpha$-Lipschitz continuous.

The metric (or nearest point) projection from $H$ onto $C$ is the mapping $P_{C}: H \rightarrow C$ which assigns to each point $x \in H$ the unique point $P_{C} x \in C$ satisfying the property

$$
\left\|x-P_{C} x\right\|=\inf _{y \in C}\|x-y\|=: d(x, C) .
$$

Some important properties of projections are gathered in the following proposition.

Proposition 3. For given $x \in H$ and $z \in C$,

(i) $z=P_{C} x \Leftrightarrow\langle x-z, y-z\rangle \leq 0, \forall y \in C$;

(ii) $z=P_{C} x \Leftrightarrow\|x-z\|^{2} \leq\|x-y\|^{2}-\|y-z\|^{2}, \forall y \in C$;

(iii) $\left\langle P_{C} x-P_{C} y, x-y\right\rangle \geq\left\|P_{C} x-P_{C} y\right\|^{2}, \forall y \in H$.

Consequently, $P_{C}$ is nonexpansive and monotone.

If $A$ is an $\alpha$-inverse-strongly monotone mapping of $C$ into $H$, then it is obvious that $A$ is $1 / \alpha$-Lipschitz continuous. We also have that if $\lambda \leq 2 \alpha$, then $I-\lambda A$ is a nonexpansive mapping from $\mathrm{C}$ to $\mathrm{H}$.

Definition 4. A mapping $T: H \rightarrow H$ is said to be

(a) nonexpansive if

$$
\|T x-T y\| \leq\|x-y\|, \quad \forall x, y \in H
$$

(b) firmly nonexpansive if $2 T-I$ is nonexpansive or, equivalently, if $T$ is 1 -inverse-strongly monotone (1ism):

$$
\langle x-y, T x-T y\rangle \geq\|T x-T y\|^{2}, \quad \forall x, y \in H
$$

alternatively, $T$ is firmly nonexpansive if and only if $T$ can be expressed as

$$
T=\frac{1}{2}(I+S),
$$

where $S: H \rightarrow H$ is nonexpansive; projections are firmly nonexpansive.

It can be easily seen that if $T$ is nonexpansive, then $I-T$ is monotone. It is also easy to see that a projection $P_{C}$ is 1-ism. Inverse-strongly monotone (also referred to as cocoercive) operators have been applied widely in solving practical problems in various fields.

Definition 5. A mapping $T: H \rightarrow H$ is said to be an averaged mapping if it can be written as the average of the identity $I$ and a nonexpansive mapping; that is,

$$
T \equiv(1-\alpha) I+\alpha S
$$

where $\alpha \in(0,1)$ and $S: H \rightarrow H$ is nonexpansive. More precisely, when the last equality holds, we say that $T$ is $\alpha$ averaged. Thus firmly nonexpansive mappings (in particular, projections) are 1/2-averaged mappings.

Proposition 6 (see [15]). Let $T: H \rightarrow H$ be a given mapping.

(i) $T$ is nonexpansive if and only if the complement $I-T$ is $1 / 2$-ism.

(ii) If $T$ is $\nu$-ism, then, for $\gamma>0, \gamma T$ is $\nu / \gamma$-ism.

(iii) $T$ is averaged if and only if the complement $I-T$ is $\nu$-ism for some $\nu>1 / 2$. Indeed, for $\alpha \in(0,1), T$ is $\alpha$-averaged if and only if $I-T$ is $1 / 2 \alpha$-ism.

Proposition 7 (see [15]). Let $S, T, V: H \rightarrow H$ be given operators.

(i) If $T=(1-\alpha) S+\alpha V$ for some $\alpha \in(0,1)$ and if $S$ is averaged and $V$ is nonexpansive, then $T$ is averaged.

(ii) $T$ is firmly nonexpansive if and only if the complement $I-T$ is firmly nonexpansive.

(iii) If $T=(1-\alpha) S+\alpha V$ for some $\alpha \in(0,1)$ and if $S$ is firmly nonexpansive and $V$ is nonexpansive, then $T$ is averaged.

(iv) The composite of finitely many averaged mappings is averaged. That is, if each of the mappings $\left\{T_{i}\right\}_{i=1}^{N}$ is averaged, then so is the composite $T_{1}, \ldots, T_{N}$. In particular, if $T_{1}$ is $\alpha_{1}$-averaged and $T_{2}$ is $\alpha_{2}$-averaged, where $\alpha_{1}, \alpha_{2} \in(0,1)$, then the composite $T_{1} T_{2}$ is $\alpha$ averaged, where $\alpha=\alpha_{1}+\alpha_{2}-\alpha_{1} \alpha_{2}$.

(v) If the mappings $\left\{T_{i}\right\}_{i=1}^{N}$ are averaged and have a common fixed point, then

$$
\bigcap_{i=1}^{N} \operatorname{Fix}\left(T_{i}\right)=\operatorname{Fix}\left(T_{1}, \ldots, T_{N}\right) .
$$

The notation Fix $(T)$ denotes the set of all fixed points of the mapping T; that is, Fix $(T)=\{x \in H: T x=x\}$. 
Proposition 8 (see [3]). Assume that $\Theta: C \times C \rightarrow \mathbf{R}$ satisfies (A1)-(A4) and let $\varphi: C \rightarrow \mathbf{R}$ be a proper lower semicontinuous and convex function. Assume that either (B1) or (B2) holds. For $r>0$ and $x \in H$, define a mapping $T_{r}^{(\Theta, \varphi)}: H \rightarrow C$ as follows:

$$
\begin{aligned}
T_{r}^{(\Theta, \varphi)}(x)=\{z \in C: & \Theta(z, y)+\varphi(y)-\varphi(z) \\
& \left.+\frac{1}{r}\langle y-z, z-x\rangle \geq 0, \forall y \in C\right\},
\end{aligned}
$$

for all $x \in H$. Then the following hold:

(i) for each $x \in H, T_{r}^{(\Theta, \varphi)}(x) \neq \emptyset$;

(ii) $T_{r}^{(\Theta, \varphi)}$ is single-valued;

(iii) $T_{r}^{(\Theta, \varphi)}$ is firmly nonexpansive; that is, for any $x, y \in H$,

$$
\left\|T_{r}^{(\Theta, \varphi)} x-T_{r}^{(\Theta, \varphi)} y\right\|^{2} \leq\left\langle T_{r}^{(\Theta, \varphi)} x-T_{r}^{(\Theta, \varphi)} y, x-y\right\rangle ;
$$

(iv) $\operatorname{Fix}\left(T_{r}^{(\Theta, \varphi)}\right)=\operatorname{MEP}(\Theta, \varphi)$;

(v) $\operatorname{MEP}(\Theta, \varphi)$ is closed and convex.

We need some facts and tools in a real Hilbert space $H$ which are listed as lemmas below.

Lemma 9. Let $X$ be a real inner product space. Then there holds the following inequality:

$$
\|x+y\|^{2} \leq\|x\|^{2}+2\langle y, x+y\rangle, \quad \forall x, y \in X .
$$

Lemma 10. Let $A: C \rightarrow H$ be a monotone mapping. In the context of the variational inequality problem the characterization of the projection (see Proposition 3(i)) implies

$$
u \in V I(C, A) \Longleftrightarrow u=P_{C}(u-\lambda A u), \quad \lambda>0 .
$$

Lemma 11 (see [16, demiclosedness principle]). Let $C$ be a nonempty closed convex subset of a real Hilbert space $H$. Let $T$ be a nonexpansive self-mapping on $C$. Then $I-T$ is demiclosed. That is, whenever $\left\{x_{n}\right\}$ is a sequence in $C$ weakly converging to some $x \in C$ and the sequence $\left\{(I-T) x_{n}\right\}$ strongly converges to some $y$, it follows that $(I-T) x=y$. Here $I$ is the identity operator of $\mathrm{H}$.
Let $\left\{T_{n}\right\}_{n=1}^{\infty}$ be an infinite family of nonexpansive mappings on $H$ and let $\left\{\lambda_{n}\right\}_{n=1}^{\infty}$ be a sequence of nonnegative numbers in $[0,1]$. For any $n \geq 1$, define a mapping $W_{n}$ on $H$ as follows:

$$
\begin{gathered}
U_{n, n+1}=I, \\
U_{n, n}=\lambda_{n} T_{n} U_{n, n+1}+\left(1-\lambda_{n}\right) I, \\
U_{n, n-1}=\lambda_{n-1} T_{n-1} U_{n, n}+\left(1-\lambda_{n-1}\right) I, \\
\vdots \\
U_{n, k}=\lambda_{k} T_{k} U_{n, k+1}+\left(1-\lambda_{k}\right) I, \\
U_{n, k-1}=\lambda_{k-1} T_{k-1} U_{n, k}+\left(1-\lambda_{k-1}\right) I,
\end{gathered}
$$

$$
\begin{gathered}
U_{n, 2}=\lambda_{2} T_{2} U_{n, 3}+\left(1-\lambda_{2}\right) I, \\
W_{n}=U_{n, 1}=\lambda_{1} T_{1} U_{n, 2}+\left(1-\lambda_{1}\right) I .
\end{gathered}
$$

Such a mapping $W_{n}$ is called the $W$-mapping generated by $T_{n}, T_{n-1}, \ldots, T_{1}$ and $\lambda_{n}, \lambda_{n-1}, \ldots, \lambda_{1}$.

Lemma 12 (see [17, Lemma 3.2]). Let $C$ be a nonempty closed convex subset of a real Hilbert space $H$. Let $\left\{T_{n}\right\}_{n=1}^{\infty}$ be a sequence of nonexpansive self-mappings on $C$ such that $\cap_{n=1}^{\infty} \operatorname{Fix}\left(T_{n}\right) \neq \emptyset$ and let $\left\{\lambda_{n}\right\}$ be a sequence in $(0, b]$ for some $b \in(0,1)$. Then, for every $x \in C$ and $k \geq 1$, the limit $\lim _{n \rightarrow \infty} U_{n, k} x$ exists, where $U_{n, k}$ is defined as in (34).

Lemma 13 (see [17, Lemma 3.3]). Let $C$ be a nonempty closed convex subset of a real Hilbert space $H$. Let $\left\{T_{n}\right\}_{n=1}^{\infty}$ be a sequence of nonexpansive self-mappings on $C$ such that $\cap_{n=1}^{\infty} F i x\left(T_{n}\right) \neq \emptyset$, and let $\left\{\lambda_{n}\right\}$ be a sequence in $(0, b]$ for some $b \in(0,1)$. Then, $\operatorname{Fix}(W)=\cap_{n=1}^{\infty} \operatorname{Fix}\left(T_{n}\right)$.

The following lemma can be easily proven, and, therefore, we omit the proof.

Lemma 14. Let $V: H \rightarrow H$ be a $\bar{\gamma}$-strongly positive bounded linear operator with constant $\bar{\gamma}>1$. Then, for $\bar{\gamma}-1>0$,

$$
\begin{array}{r}
\langle(V-I) x-(V-I) y, x-y\rangle \geq(\bar{\gamma}-1)\|x-y\|^{2}, \\
\forall x, y \in H .
\end{array}
$$

That is, $V-I$ is strongly monotone with constant $\bar{\gamma}-1$.

Let $C$ be a nonempty closed convex subset of a real Hilbert space $H$. We introduce some notations. Let $\lambda$ be a number in $(0,1]$ and let $\mu>0$. Associating with a nonexpansive mapping $T: C \rightarrow H$, we define the mapping $T^{\lambda}: C \rightarrow H$ by

$$
T^{\lambda} x:=T x-\lambda \mu F(T x), \quad \forall x \in C,
$$

where $F: H \rightarrow H$ is an operator such that, for some positive constants $\kappa, \eta>0, F$ is $\kappa$-Lipschitzian and $\eta$-strongly monotone on $H$; that is, $F$ satisfies the following conditions:

$$
\|F x-F y\| \leq \kappa\|x-y\|, \quad\langle F x-F y, x-y\rangle \geq \eta\|x-y\|^{2},
$$

for all $x, y \in H$. 
Lemma 15 (see [18, Lemma 3.1]). $T^{\lambda}$ is a contraction provided $0<\mu<2 \eta / \kappa^{2}$; that is,

$$
\left\|T^{\lambda} x-T^{\lambda} y\right\| \leq(1-\lambda \tau)\|x-y\|, \quad \forall x, y \in C,
$$

where $\tau=1-\sqrt{1-\mu\left(2 \eta-\mu \kappa^{2}\right)} \in(0,1]$.

Lemma 16 ([10, Lemma 2.5]). Let $H$ be a real Hilbert space. Given a nonempty closed convex subset of $H$ and points $x, y, z \in H$ and given also a real number $a \in \mathbf{R}$, the set

$$
\left\{v \in C:\|y-v\|^{2} \leq\|x-v\|^{2}+\langle z, v\rangle+a\right\}
$$

is convex (and closed).

Recall that a set-valued mapping $T: D(T) \subset H \rightarrow 2^{H}$ is called monotone if, for all $x, y \in D(T), f \in T x$ and $g \in T y$ imply

$$
\langle f-g, x-y\rangle \geq 0 \text {. }
$$

A set-valued mapping $T$ is called maximal monotone if $T$ is monotone and $(I+\lambda T) D(T)=H$ for each $\lambda>0$, where $I$ is the identity mapping of $H$. We denote by $G(T)$ the graph of $T$. It is known that a monotone mapping $T$ is maximal if and only if, for $(x, f) \in H \times H,\langle f-g, x-y\rangle \geq 0$ for every $(y, g) \in G(T)$ implies $f \in T x$. Let $A: C \rightarrow H$ be a monotone, $k$-Lipschitzcontinuous mapping and let $N_{C} v$ be the normal cone to $C$ at $v \in C$; that is,

$$
N_{C} v=\{w \in H:\langle v-u, w\rangle \geq 0, \forall u \in C\} .
$$

Define

$$
T v= \begin{cases}A v+N_{C} v, & \text { if } v \in C, \\ \emptyset, & \text { if } v \notin C .\end{cases}
$$

Then, $T$ is maximal monotone and $0 \in T v$ if and only if $v \in$ $\mathrm{VI}(C, A)$.

Lemma 17 ([10, Lemma 2.6]). Let $C$ be a nonempty subset of a Hilbert space $H$ and let $S: C \rightarrow C$ be an asymptotically $k$-strict pseudocontractive mapping in the intermediate sense with sequence $\left\{\gamma_{n}\right\}$. Then

$$
\begin{aligned}
& \left\|S^{n} x-S^{n} y\right\| \\
& \leq \frac{1}{1-k} \\
& \quad \times\left(k\|x-y\|+\sqrt{\left(1+(1-k) \gamma_{n}\right)\|x-y\|^{2}+(1-k) c_{n}}\right)
\end{aligned}
$$

for all $x, y \in C$ and $n \geq 1$.

Lemma 18 ([10, Lemma 2.7]). Let $C$ be a nonempty subset of a Hilbert space $H$ and let $S: C \rightarrow C$ be a uniformly continuous asymptotically $k$-strict pseudocontractive mapping in the intermediate sense with sequence $\left\{\gamma_{n}\right\}$. Let $\left\{x_{n}\right\}$ be a sequence in $C$ such that $\left\|x_{n}-x_{n+1}\right\| \rightarrow 0$ and $\left\|x_{n}-S^{n} x_{n}\right\| \rightarrow 0$ as $n \rightarrow \infty$. Then $\left\|x_{n}-S x_{n}\right\| \rightarrow 0$ as $n \rightarrow \infty$.
Lemma 19 (demiclosedness principle [10, Proposition 3.1]). Let $C$ be a nonempty closed convex subset of a Hilbert space $H$ and let $S: C \rightarrow C$ be a continuous asymptotically $k$-strict pseudocontractive mapping in the intermediate sense with sequence $\left\{\gamma_{n}\right\}$. Then $I-S$ is demiclosed at zero in the sense that if $\left\{x_{n}\right\}$ is a sequence in $C$ such that $x_{n} \rightarrow x \in C$ and $\lim \sup _{m \rightarrow \infty} \lim \sup _{n \rightarrow \infty}\left\|x_{n}-S^{m} x_{n}\right\|=0$, then $(I-S) x=0$.

Lemma 20 ([10, Proposition 3.2]). Let $C$ be a nonempty closed convex subset of a Hilbert space $H$ and let $S: C \rightarrow$ $C$ be a continuous asymptotically $k$-strict pseudocontractive mapping in the intermediate sense with sequence $\left\{\gamma_{n}\right\}$ such that Fix $(S) \neq \emptyset$. Then Fix $(S)$ is closed and convex.

Remark 21. Lemmas 19 and 20 give some basic properties of an asymptotically $k$-strict pseudocontractive mapping in the intermediate sense with sequence $\left\{\gamma_{n}\right\}$.

Lemma 22 (see [19]). Let $C$ be a closed convex subset of a real Hilbert space $H$. Let $\left\{x_{n}\right\}$ be a sequence in $H$ and $u \in H$. Let $q=P_{C} u$. If $\left\{x_{n}\right\}$ is such that $\omega_{w}\left(x_{n}\right) \subset C$ and satisfies the condition

$$
\left\|x_{n}-u\right\| \leq\|u-q\|, \quad \forall n
$$

then $x_{n} \rightarrow$ qas $n \rightarrow \infty$.

Lemma 23. Let $H$ be a real Hilbert space. Then the following hold:

(a) $\|x-y\|^{2}=\|x\|^{2}-\|y\|^{2}-2\langle x-y, y\rangle$ for all $x, y \in H$;

(b) $\|\lambda x+\mu y\|^{2}=\lambda\|x\|^{2}+\mu\|y\|^{2}-\lambda \mu\|x-y\|^{2}$ for all $x, y \epsilon$ $H$ and $\lambda, \mu \in[0,1]$ with $\lambda+\mu=1$;

(c) if $\left\{x_{n}\right\}$ is a sequence in $H$ such that $x_{n} \rightarrow x$, it follows that

$\limsup _{n \rightarrow \infty}\left\|x_{n}-y\right\|^{2}=\limsup _{n \rightarrow \infty}\left\|x_{n}-x\right\|^{2}+\|x-y\|^{2}, \quad \forall y \in H$.

\section{Convex Minimization Problems with Constraints}

In this section, we will introduce and analyze one iterative algorithm by hybrid shrinking projection method for finding a solution of the CMP (13) with constraints of several problems: finitely many GMEPs, finitely many VIPs, GSVI (8), and the fixed point problem of an asymptotically strict pseudocontractive mapping in the intermediate sense in a real Hilbert space. We prove strong convergence theorem for the iterative algorithm under suitable conditions. This iterative algorithm is based on shrinking projection method, Korpelevich's extragradient method, hybrid steepest-descent method in [7], viscosity approximation method, averaged mapping approach to the GPA in [14], and strongly positive bounded linear operator technique.

Theorem 24. Let $C$ be a nonempty closed convex subset of a real Hilbert space $H$. Let $M, N$ be two integers. Let $f: C \rightarrow \mathbf{R}$ be a convex functional with L-Lipschitz 
continuous gradient $\nabla f$. Let $\Theta_{k}$ be a bifunction from $C \times C$ to $\mathbf{R}$ satisfying (A1)-(A4) and let $\varphi_{k}: C \rightarrow \mathbf{R} \cup\{+\infty\}$ be a proper lower semicontinuous and convex function, where $k \in\{1,2, \ldots, M\}$. Let $B_{k}, A_{i}: H \rightarrow H$, and $F_{j}: C \rightarrow H$ be $\mu_{k}$-inverse-strongly monotone, $\eta_{i}$-inverse-strongly monotone, and $\zeta_{j}$-inverse-strongly monotone, respectively, where $k \in$ $\{1,2, \ldots, M\}, i \in\{1,2, \ldots, N\}$, and $j \in\{1,2\}$. Let $S$ : $C \rightarrow C$ be a uniformly continuous asymptotically $k$-strict pseudocontractive mapping in the intermediate sense for some $0 \leq k<1$ with sequence $\left\{\gamma_{n}\right\} \subset[0, \infty)$ such that $\lim _{n \rightarrow \infty} \gamma_{n}=$ 0 and $\left\{c_{n}\right\} \subset[0, \infty)$ such that $\lim _{n \rightarrow \infty} c_{n}=0$. Let $V$ be $a \bar{\gamma}-$ strongly positive bounded linear operator with $\bar{\gamma}>1$. Let $F$ : $H \rightarrow H$ be a $\kappa$-Lipschitzian and $\eta$-strongly monotone operator with positive constants $\kappa, \eta>0$. Let $Q: H \rightarrow H$ be an $l$ Lipschitzian mapping with constant $l \geq 0$. Let $0<\mu<2 \eta / \kappa^{2}$ and $0 \leq \gamma l \leq \tau$, where $\tau=1-\sqrt{1-\mu\left(2 \eta-\mu \kappa^{2}\right)}$. Assume that $\Omega:=\cap_{k=1}^{M} G M E P\left(\Theta_{k}, \varphi_{k}, B_{k}\right) \cap \cap_{i=1}^{N} \operatorname{VI}\left(C, A_{i}\right) \cap \operatorname{GSVI}(G) \cap$ $\operatorname{Fix}(S) \cap \Gamma$ is nonempty and bounded and that either (B1) or (B2) holds. Let $0<\alpha \leq \alpha_{n} \leq 1, k \leq \delta_{n} \leq d<1$ for all $n \geq 1$, and let $\left\{\beta_{n}\right\},\left\{\sigma_{n}\right\}$ be sequences in $(0,1]$. Pick any $x_{0} \in H$ and set $C_{1}=C, x_{1}=P_{C_{1}} x_{0}$. Let $\left\{x_{n}\right\}$ be a sequence generated by the following algorithm:

$$
\begin{gathered}
u_{n}=T_{r_{M, n}}^{\left(\Theta_{M}, \varphi_{M}\right)}\left(I-r_{M, n} B_{M}\right) T_{r_{M-1, n}}^{\left(\Theta_{M-1}, \varphi_{M-1}\right)} \\
\quad \times\left(I-r_{M-1, n} B_{M-1}\right) \cdots T_{r_{1, n}}^{\left(\Theta_{1}, \varphi_{1}\right)}\left(I-r_{1, n} B_{1}\right) x_{n} \\
v_{n}=P_{C}\left(I-\lambda_{N, n} A_{N}\right) P_{C}\left(I-\lambda_{N-1, n} A_{N-1}\right) \cdots P_{C} \\
\times\left(I-\lambda_{2, n} A_{2}\right) P_{C}\left(I-\lambda_{1, n} A_{1}\right) u_{n}, \\
z_{n}=\beta_{n} x_{n}+\left(\left(1-\beta_{n}\right) I-s_{n} V\right) T_{n} G v_{n} \\
\quad+s_{n}\left[T_{n} x_{n}-\sigma_{n}\left(\mu F\left(T_{n} x_{n}\right)-\gamma Q x_{n}\right)\right], \\
k_{n}=\delta_{n} z_{n}+\left(1-\delta_{n}\right) S^{n} z_{n}, \\
y_{n}=\left(1-\alpha_{n}\right) x_{n}+\alpha_{n} k_{n}, \\
C_{n+1}=\left\{z \in C_{n}:\left\|y_{n}-z\right\|^{2} \leq\left\|x_{n}-z\right\|^{2}+\theta_{n}\right\}, \\
x_{n+1}=P_{C_{n+1}} x_{0}, \\
\forall n \geq 1,
\end{gathered}
$$

where $P_{C}\left(I-\lambda_{n} \nabla f\right)=s_{n} I+\left(1-s_{n}\right) T_{n}$ (here $T_{n}$ is nonexpansive; $s_{n}=\left(2-\lambda_{n} L\right) / 4 \in(0,1 / 2)$ for each $\left.\lambda_{n} \in(0,2 / L)\right), \theta_{n}=$ $\left(s_{n}+\gamma_{n}\right)\left(1+\gamma_{n}\right) \Delta_{n}+c_{n}$, and $\Delta_{n}=\sup \left\{\left\|x_{n}-p\right\|^{2}+(\|(I-\right.$ $\left.V) p\|+\|(\gamma Q-\mu F) p \|)^{2} /(\bar{\gamma}-1): p \in \Omega\right\}<\infty$. Suppose that the following conditions are satisfied:

(i) $s_{n} \in(0,1 / 2)$ for each $\lambda_{n} \in(0,2 / L), \lim _{n \rightarrow \infty} s_{n}=0(\Leftrightarrow$ $\left.\lim _{n \rightarrow \infty} \lambda_{n}=2 / L\right)$;

(ii) $\left\{r_{k, n}\right\} \subset\left[e_{k}, f_{k}\right] \subset\left(0,2 \mu_{k}\right),\left\{\lambda_{i, n}\right\} \subset\left[a_{i}, b_{i}\right] \subset\left(0,2 \eta_{i}\right)$, and $v_{j} \in\left(0,2 \zeta_{j}\right)$, where $k \in\{1,2, \ldots, M\}, i \in$ $\{1,2, \ldots, N\}$, and $j \in\{1,2\}$;

(iii) $0<\liminf _{n \rightarrow \infty} \beta_{n} \leq \limsup _{n \rightarrow \infty} \beta_{n}<1$.
Then one has the following:

(I) $\left\{x_{n}\right\}$ converges strongly as $\lambda_{n} \rightarrow 2 / L\left(\Leftrightarrow s_{n} \rightarrow 0\right)$ to $x^{*}=P_{\Omega} x_{0}$

(II) $\left\{x_{n}\right\}$ converges strongly as $\lambda_{n} \rightarrow 2 / L\left(\Leftrightarrow s_{n} \rightarrow\right.$ 0) to $x^{*}=P_{\Omega} x_{0}$ provided $\left\|x_{n}-z_{n}\right\|=o\left(s_{n}\right)$ and $\lim _{n \rightarrow \infty} \sigma_{n}=0$, which is the unique solution in $\Omega$ to the VIP

$$
\left\langle(I-V) x^{*}, p-x^{*}\right\rangle \leq 0, \quad \forall p \in \Omega .
$$

Equivalently, $x^{*}=P_{\Omega}(2 I-V) x^{*}$.

Proof. Since $\nabla f$ is $L$-Lipschitzian, it follows that $\nabla f$ is $1 / L$ ism. By Proposition 6(ii) we know that, for $\lambda>0, \lambda \nabla f$ is $1 / \lambda L$-ism. So by Proposition 6(iii) we deduce that $I-\lambda \nabla f$ is $\lambda L / 2$-averaged. Now since the projection $P_{C}$ is $1 / 2$-averaged, it is easy to see from Proposition 7 (iv) that the composite $P_{C}(I-\lambda \nabla f)$ is $(2+\lambda L) / 4$-averaged for $\lambda \in(0,2 / L)$. Hence we obtain that, for each $n \geq 1, P_{C}\left(I-\lambda_{n} \nabla f\right)$ is $\left(2+\lambda_{n} L\right) / 4$ averaged for each $\lambda_{n} \in(0,2 / L)$. Therefore, we can write

$$
\begin{aligned}
P_{C}\left(I-\lambda_{n} \nabla f\right) & =\frac{2-\lambda_{n} L}{4} I+\frac{2+\lambda_{n} L}{4} T_{n} \\
& =s_{n} I+\left(1-s_{n}\right) T_{n},
\end{aligned}
$$

where $T_{n}$ is nonexpansive and $s_{n}:=s_{n}\left(\lambda_{n}\right)=\left(2-\lambda_{n} L\right) / 4 \epsilon$ $(0,1 / 2)$ for each $\lambda_{n} \in(0,2 / L)$. It is clear that

$$
\lambda_{n} \longrightarrow \frac{2}{L} \Longleftrightarrow s_{n} \longrightarrow 0 .
$$

As $\lim _{n \rightarrow \infty} s_{n}=0$ and $0<\liminf _{n \rightarrow \infty} \beta_{n} \leq$ limsup $\operatorname{sum}_{n \rightarrow \infty} \beta_{n}<1$, we may assume, without loss of generality, that $\left\{\beta_{n}\right\} \subset[a, \widehat{a}] \subset(0,1)$ and $\beta_{n}+s_{n}\|V\| \leq 1$ for all $n \geq 1$. Since $V$ is a $\bar{\gamma}$-strongly positive bounded linear operator on $H$, we know that

$$
\|V\|=\sup \{\langle V u, u\rangle: u \in H,\|u\|=1\} \geq \bar{\gamma}>1 .
$$

Taking into account that $\beta_{n}+s_{n}\|V\| \leq 1$ for all $n \geq 1$, we have

$$
\begin{aligned}
\left\langle\left(\left(1-\beta_{n}\right) I-s_{n} V\right) u, u\right\rangle & =1-\beta_{n}-s_{n}\langle V u, u\rangle \\
& \geq 1-\beta_{n}-s_{n}\|V\| \geq 0 ;
\end{aligned}
$$

that is, $\left(1-\beta_{n}\right) I-s_{n} V$ is positive. It follows that

$$
\begin{aligned}
\|(1- & \left.\beta_{n}\right) I-s_{n} V \| \\
& =\sup \left\{\left\langle\left(\left(1-\beta_{n}\right) I-s_{n} V\right) u, u\right\rangle: u \in H,\|u\|=1\right\} \\
& =\sup \left\{1-\beta_{n}-s_{n}\langle V u, u\rangle: u \in H,\|u\|=1\right\} \\
& \leq 1-\beta_{n}-s_{n} \bar{\gamma} .
\end{aligned}
$$

Put

$$
\begin{aligned}
\Delta_{n}^{k}= & T_{r_{k, n}}^{\left(\Theta_{k}, \varphi_{k}\right)}\left(I-r_{k, n} B_{k}\right) T_{r_{k-1, n}}^{\left(\Theta_{k-1}, \varphi_{k-1}\right)} \\
& \times\left(I-r_{k-1, n} B_{k-1}\right) \cdots T_{r_{1, n}}^{\left(\Theta_{1}, \varphi_{1}\right)}\left(I-r_{1, n} B_{1}\right) x_{n}
\end{aligned}
$$


for all $k \in\{1,2, \ldots, M\}$ and $n \geq 1$ and

$$
\Lambda_{n}^{i}=P_{C}\left(I-\lambda_{i, n} B_{i}\right) P_{C}\left(I-\lambda_{i-1, n} B_{i-1}\right) \cdots P_{C}\left(I-\lambda_{1, n} B_{1}\right)
$$

for all $i \in\{1,2, \ldots, N\}, \Delta_{n}^{0}=I$, and $\Lambda_{n}^{0}=I$, where $I$ is the identity mapping on $H$. Then we have that $u_{n}=\Delta_{n}^{M} x_{n}$ and $v_{n}=\Lambda_{n}^{N} u_{n}$.

We divide the rest of the proof into several steps.

Step 1. We show that $\left\{x_{n}\right\}$ is well defined. It is obvious that $C_{n}$ is closed and convex. As the defining inequality in $C_{n}$ is equivalent to the inequality

$$
\left\langle 2\left(x_{n}-y_{n}\right), z\right\rangle \leq\left\|x_{n}\right\|^{2}-\left\|y_{n}\right\|^{2}+\theta_{n}
$$

by Lemma 16 we know that $C_{n}$ is convex for every $n \geq 1$.

First of all, let us show that $\Omega \subset C_{n}$ for all $n \geq 1$. Suppose that $\Omega \subset C_{n}$ for some $n \geq 1$. Take $p \in \Omega$ arbitrarily. From (46) and Proposition 8(iii), we have

$$
\begin{aligned}
& \left\|u_{n}-p\right\| \\
& =\| T_{r_{M, n}}^{\left(\Theta_{M}, \varphi_{M}\right)}\left(I-r_{M, n} B_{M}\right) \Delta_{n}^{M-1} x_{n} \\
& -T_{r_{M, n}}^{\left(\Theta_{M}, \varphi_{M}\right)}\left(I-r_{M, n} B_{M}\right) \Delta_{n}^{M-1} p \| \\
& \leq\left\|\left(I-r_{M, n} B_{M}\right) \Delta_{n}^{M-1} x_{n}-\left(I-r_{M, n} B_{M}\right) \Delta_{n}^{M-1} p\right\| \\
& \leq\left\|\Delta_{n}^{M-1} x_{n}-\Delta_{n}^{M-1} p\right\| \\
& \leq\left\|\Delta_{n}^{0} x_{n}-\Delta_{n}^{0} p\right\| \\
& =\left\|x_{n}-p\right\| \text {. }
\end{aligned}
$$

Similarly, we have

$$
\begin{aligned}
& \left\|v_{n}-p\right\| \\
& \quad=\left\|P_{C}\left(I-\lambda_{N, n} A_{N}\right) \Lambda_{n}^{N-1} u_{n}-P_{C}\left(I-\lambda_{N, n} A_{N}\right) \Lambda_{n}^{N-1} p\right\| \\
& \quad \leq\left\|\left(I-\lambda_{N, n} A_{N}\right) \Lambda_{n}^{N-1} u_{n}-\left(I-\lambda_{N, n} A_{N}\right) \Lambda_{n}^{N-1} p\right\| \\
& \quad \leq\left\|\Lambda_{n}^{N-1} u_{n}-\Lambda_{n}^{N-1} p\right\| \\
& \quad \vdots \\
& \quad \leq\left\|\Lambda_{n}^{0} u_{n}-\Lambda_{n}^{0} p\right\| \\
& \quad=\left\|u_{n}-p\right\| .
\end{aligned}
$$

Combining (56) and (57), we have

$$
\left\|v_{n}-p\right\| \leq\left\|x_{n}-p\right\| .
$$

Since $p=G p=P_{C}\left(I-v_{1} F_{1}\right) P_{C}\left(I-v_{2} F_{2}\right) p, F_{j}$ is $\zeta_{j}$-inversestrongly monotone for $j=1,2$, and $0 \leq \nu_{j} \leq 2 \zeta_{j}$ for $j=1,2$, we deduce that, for any $n \geq 1$,

$$
\begin{aligned}
& \left\|G v_{n}-p\right\|^{2} \\
& =\| P_{C}\left(I-v_{1} F_{1}\right) P_{C}\left(I-v_{2} F_{2}\right) v_{n} \\
& -P_{C}\left(I-v_{1} F_{1}\right) P_{C}\left(I-v_{2} F_{2}\right) p \|^{2} \\
& \leq \|\left(I-v_{1} F_{1}\right) P_{C}\left(I-v_{2} F_{2}\right) v_{n} \\
& -\left(I-v_{1} F_{1}\right) P_{C}\left(I-v_{2} F_{2}\right) p \|^{2} \\
& =\|\left[P_{C}\left(I-v_{2} F_{2}\right) v_{n}-P_{C}\left(I-v_{2} F_{2}\right) p\right] \\
& -v_{1}\left[F_{1} P_{C}\left(I-v_{2} F_{2}\right) v_{n}-F_{1} P_{C}\left(I-v_{2} F_{2}\right) p\right] \|^{2} \\
& \leq\left\|P_{C}\left(I-v_{2} F_{2}\right) v_{n}-P_{C}\left(I-v_{2} F_{2}\right) p\right\|^{2} \\
& +v_{1}\left(\nu_{1}-2 \zeta_{1}\right) \\
& \times\left\|F_{1} P_{C}\left(I-v_{2} F_{2}\right) v_{n}-F_{1} P_{C}\left(I-v_{2} F_{2}\right) p\right\|^{2} \\
& \leq\left\|P_{C}\left(I-v_{2} F_{2}\right) v_{n}-P_{C}\left(I-v_{2} F_{2}\right) p\right\|^{2} \\
& \leq\left\|\left(I-v_{2} F_{2}\right) v_{n}-\left(I-v_{2} F_{2}\right) p\right\|^{2} \\
& =\left\|\left(v_{n}-p\right)-v_{2}\left(F_{2} v_{n}-F_{2} p\right)\right\|^{2} \\
& \leq\left\|v_{n}-p\right\|^{2}+v_{2}\left(v_{2}-2 \zeta_{2}\right)\left\|F_{2} v_{n}-F_{2} p\right\|^{2} \\
& \leq\left\|v_{n}-p\right\|^{2} \text {. }
\end{aligned}
$$

Utilizing Lemma 15, from (46), (52), (58), and (59), we obtain that

$$
\begin{aligned}
& \left\|z_{n}-p\right\| \\
& =\| \beta_{n}\left(x_{n}-p\right)+\left(\left(1-\beta_{n}\right) I-s_{n} V\right)\left(T_{n} G v_{n}-p\right) \\
& \quad+s_{n}\left[T_{n} x_{n}-\sigma_{n}\left(\mu F\left(T_{n} x_{n}\right)-\gamma Q x_{n}\right)-p\right] \\
& \quad+s_{n}(I-V) p \| \\
& =\| \beta_{n}\left(x_{n}-p\right)+\left(\left(1-\beta_{n}\right) I-s_{n} V\right)\left(T_{n} G v_{n}-p\right) \\
& +s_{n}\left[\sigma_{n} \gamma\left(Q x_{n}-Q p\right)+\left(I-\sigma_{n} \mu F\right) T_{n} x_{n}\right. \\
& \left.\quad-\left(I-\sigma_{n} \mu F\right) T_{n} p\right] \\
& +s_{n}\left[(I-V) p+\sigma_{n}(\gamma Q-\mu F) p\right] \| \\
& \leq \beta_{n}\left\|x_{n}-p\right\|+\left\|\left(1-\beta_{n}\right) I-s_{n} V\right\|\left\|T_{n} G v_{n}-p\right\| \\
& +s_{n}\left[\sigma_{n} \gamma\left\|Q x_{n}-Q p\right\|\right. \\
& \left.\quad+\left\|\left(I-\sigma_{n} \mu F\right) T_{n} x_{n}-\left(I-\sigma_{n} \mu F\right) T_{n} p\right\|\right] \\
& +s_{n}\left\|(I-V) p+\sigma_{n}(\gamma Q-\mu F) p\right\| \\
& \leq \beta_{n}\left\|x_{n}-p\right\|+\left(1-\beta_{n}-s_{n} \bar{\gamma}\right)\left\|G v_{n}-p\right\| \\
& +s_{n}\left[\sigma_{n} \gamma l\left\|x_{n}-p\right\|+\left(1-\sigma_{n} \tau\right)\left\|x_{n}-p\right\|\right]
\end{aligned}
$$




$$
\begin{aligned}
& +s_{n}(\|(I-V) p\|+\|(\gamma Q-\mu F) p\|) \\
\leq & \beta_{n}\left\|x_{n}-p\right\|+\left(1-\beta_{n}-s_{n} \bar{\gamma}\right)\left\|v_{n}-p\right\| \\
& +s_{n}\left(1-\sigma_{n}(\tau-\gamma l)\right)\left\|x_{n}-p\right\| \\
& +s_{n}(\|(I-V) p\|+\|(\gamma Q-\mu F) p\|) \\
\leq & \beta_{n}\left\|x_{n}-p\right\|+\left(1-\beta_{n}-s_{n} \bar{\gamma}\right)\left\|x_{n}-p\right\| \\
& +s_{n}\left(1-\sigma_{n}(\tau-\gamma l)\right)\left\|x_{n}-p\right\| \\
& +s_{n}(\|(I-V) p\|+\|(\gamma Q-\mu F) p\|) \\
= & \left(1-s_{n} \bar{\gamma}\right)\left\|x_{n}-p\right\|+s_{n}\left(1-\sigma_{n}(\tau-\gamma l)\right)\left\|x_{n}-p\right\| \\
& +s_{n}(\|(I-V) p\|+\|(\gamma Q-\mu F) p\|) \\
\leq & \left(1-s_{n} \bar{\gamma}\right)\left\|x_{n}-p\right\|+s_{n}\left\|x_{n}-p\right\| \\
& +s_{n}(\|(I-V) p\|+\|(\gamma Q-\mu F) p\|) \\
= & \left(1-s_{n}(\bar{\gamma}-1)\right)\left\|x_{n}-p\right\| \\
& +s_{n}(\|(I-V) p\|+\|(\gamma Q-\mu F) p\|) \\
= & \left(1-s_{n}(\bar{\gamma}-1)\right)\left\|x_{n}-p\right\| \\
& +s_{n}(\bar{\gamma}-1) \frac{\|(I-V) p\|+\|(\gamma Q-\mu F) p\|}{\bar{\gamma}-1}
\end{aligned}
$$

which hence yields

$$
\begin{aligned}
\| z_{n}- & p \|^{2} \\
\leq & \left(1-s_{n}(\bar{\gamma}-1)\right)\left\|x_{n}-p\right\|^{2}+s_{n}(\bar{\gamma}-1) \\
& \times \frac{(\|(I-V) p\|+\|(\gamma Q-\mu F) p\|)^{2}}{(\bar{\gamma}-1)^{2}} \\
\leq & \left\|x_{n}-p\right\|^{2}+s_{n} \frac{(\|(I-V) p\|+\|(\gamma Q-\mu F) p\|)^{2}}{\bar{\gamma}-1} .
\end{aligned}
$$

By Lemma 23(b), we deduce from (46) and (61) that

$$
\begin{aligned}
\| k_{n}- & p \|^{2} \\
= & \left\|\delta_{n}\left(z_{n}-p\right)+\left(1-\delta_{n}\right)\left(S^{n} z_{n}-p\right)\right\|^{2} \\
= & \delta_{n}\left\|z_{n}-p\right\|^{2}+\left(1-\delta_{n}\right)\left\|S^{n} z_{n}-p\right\|^{2} \\
& -\delta_{n}\left(1-\delta_{n}\right)\left\|z_{n}-S^{n} z_{n}\right\|^{2} \\
\leq & \delta_{n}\left\|z_{n}-p\right\|^{2}+\left(1-\delta_{n}\right) \\
& \times\left[\left(1+\gamma_{n}\right)\left\|z_{n}-p\right\|^{2}+k\left\|z_{n}-S^{n} z_{n}\right\|^{2}+c_{n}\right] \\
& -\delta_{n}\left(1-\delta_{n}\right)\left\|z_{n}-S^{n} z_{n}\right\|^{2} \\
= & {\left[1+\gamma_{n}\left(1-\delta_{n}\right)\right]\left\|z_{n}-p\right\|^{2} } \\
& +\left(1-\delta_{n}\right)\left(k-\delta_{n}\right)\left\|z_{n}-S^{n} z_{n}\right\|^{2}+\left(1-\delta_{n}\right) c_{n}
\end{aligned}
$$

$$
\begin{aligned}
& \leq\left(1+\gamma_{n}\right)\left\|z_{n}-p\right\|^{2} \\
& \quad+\left(1-\delta_{n}\right)\left(k-\delta_{n}\right)\left\|z_{n}-S^{n} z_{n}\right\|^{2}+c_{n} \\
& \leq\left(1+\gamma_{n}\right)\left\|z_{n}-p\right\|^{2}+c_{n} \\
& \leq\left(1+\gamma_{n}\right)\left(\left\|x_{n}-p\right\|^{2}\right. \\
& \left.\quad+s_{n} \frac{(\|(I-V) p\|+\|(\gamma Q-\mu F) p\|)^{2}}{\bar{\gamma}-1}\right)+c_{n} .
\end{aligned}
$$

So, from (46) and (62) we get

$$
\begin{aligned}
& \left\|y_{n}-p\right\|^{2} \\
& =\left\|\left(1-\alpha_{n}\right)\left(x_{n}-p\right)+\alpha_{n}\left(k_{n}-p\right)\right\|^{2} \\
& \leq\left(1-\alpha_{n}\right)\left\|x_{n}-p\right\|^{2}+\alpha_{n}\left\|k_{n}-p\right\|^{2} \\
& \leq\left(1-\alpha_{n}\right)\left\|x_{n}-p\right\|^{2}+\alpha_{n} \\
& \quad \times\left[( 1 + \gamma _ { n } ) \left(\left\|x_{n}-p\right\|^{2}\right.\right. \\
& \left.\left.\quad+s_{n} \frac{\left(\|(I-V) p\|+\|(\gamma Q-\mu F) p\|^{2}\right.}{\bar{\gamma}-1}\right)+c_{n}\right]
\end{aligned}
$$

$\leq\left(1+\gamma_{n}\right)$

$$
\times\left(\left\|x_{n}-p\right\|^{2}+s_{n} \frac{(\|(I-V) p\|+\|(\gamma Q-\mu F) p\|)^{2}}{\bar{\gamma}-1}\right)
$$$$
+c_{n}
$$

$$
=\left\|x_{n}-p\right\|^{2}+\gamma_{n}\left\|x_{n}-p\right\|^{2}+\left(1+\gamma_{n}\right) s_{n}
$$

$$
\times \frac{(\|(I-V) p\|+\|(\gamma Q-\mu F) p\|)^{2}}{\bar{\gamma}-1}+c_{n}
$$

$\leq\left\|x_{n}-p\right\|^{2}+\gamma_{n}\left(1+\gamma_{n}\right)\left\|x_{n}-p\right\|^{2}+s_{n}\left(1+\gamma_{n}\right)$

$\times \frac{(\|(I-V) p\|+\|(\gamma Q-\mu F) p\|)^{2}}{\bar{\gamma}-1}+c_{n}$

$\leq\left\|x_{n}-p\right\|^{2}+\left(\gamma_{n}+s_{n}\right)\left(1+\gamma_{n}\right)\left\|x_{n}-p\right\|^{2}$

$+\left(s_{n}+\gamma_{n}\right)\left(1+\gamma_{n}\right) \frac{(\|(I-V) p\|+\|(\gamma Q-\mu F) p\|)^{2}}{\bar{\gamma}-1}$

$+c_{n}$

$=\left\|x_{n}-p\right\|^{2}+\left(s_{n}+\gamma_{n}\right)\left(1+\gamma_{n}\right)$

$\times\left(\left\|x_{n}-p\right\|^{2}+\frac{(\|(I-V) p\|+\|(\gamma Q-\mu F) p\|)^{2}}{\bar{\gamma}-1}\right)$

$+c_{n}$ 


$$
\begin{aligned}
& \leq\left\|x_{n}-p\right\|^{2}+\left(s_{n}+\gamma_{n}\right)\left(1+\gamma_{n}\right) \Delta_{n}+c_{n} \\
& =\left\|x_{n}-p\right\|^{2}+\theta_{n},
\end{aligned}
$$

where $\theta_{n}=\left(s_{n}+\gamma_{n}\right)\left(1+\gamma_{n}\right) \Delta_{n}+c_{n}$ and $\Delta_{n}=\sup \left\{\left\|x_{n}-p\right\|^{2}+\right.$ $\left.(\|(I-V) p\|+\|(\gamma Q-\mu F) p\|)^{2} /(\bar{\gamma}-1): p \in \Omega\right\}<\infty$. Hence $p \in C_{n+1}$. This implies that $\Omega \subset C_{n}$ for all $n \geq 1$. Therefore, $\left\{x_{n}\right\}$ is well defined.

Step 2. We prove that $\left\|x_{n}-k_{n}\right\| \rightarrow 0,\left\|x_{n}-z_{n}\right\| \rightarrow 0$ and $\left\|S^{n} z_{n}-z_{n}\right\| \rightarrow 0$ as $n \rightarrow \infty$.

Indeed, let $x^{*}=P_{\Omega} x_{0}$. From $x_{n}=P_{C_{n}} x_{0}$ and $x^{*} \in \Omega \subset$ $C_{n}$, we obtain

$$
\left\|x_{n}-x_{0}\right\| \leq\left\|x^{*}-x_{0}\right\|
$$

This implies that $\left\{x_{n}\right\}$ is bounded and hence $\left\{u_{n}\right\},\left\{v_{n}\right\}$, $\left\{z_{n}\right\},\left\{k_{n}\right\}$, and $\left\{y_{n}\right\}$ are also bounded. Since $x_{n+1} \in C_{n+1} \subset C_{n}$ and $x_{n}=P_{C_{n}} x_{0}$, we have

$$
\left\|x_{n}-x_{0}\right\| \leq\left\|x_{n+1}-x_{0}\right\|, \quad \forall n \geq 1
$$

Therefore $\lim _{n \rightarrow \infty}\left\|x_{n}-x_{0}\right\|$ exists. From $x_{n}=P_{C_{n}} x_{0}, x_{n+1} \in$ $C_{n+1} \subset C_{n}$, by Proposition 3(ii), we obtain

$$
\left\|x_{n+1}-x_{n}\right\|^{2} \leq\left\|x_{0}-x_{n+1}\right\|^{2}-\left\|x_{0}-x_{n}\right\|^{2}
$$

which implies

$$
\lim _{n \rightarrow \infty}\left\|x_{n+1}-x_{n}\right\|=0
$$

It follows from $x_{n+1} \in C_{n+1}$ that $\left\|y_{n}-x_{n+1}\right\|^{2} \leq\left\|x_{n}-x_{n+1}\right\|^{2}+$ $\theta_{n}$ and hence

$$
\begin{aligned}
\| x_{n}- & y_{n} \|^{2} \\
& \leq 2\left(\left\|x_{n}-x_{n+1}\right\|^{2}+\left\|x_{n+1}-y_{n}\right\|^{2}\right) \\
& \leq 2\left(\left\|x_{n}-x_{n+1}\right\|^{2}+\left\|x_{n}-x_{n+1}\right\|^{2}+\theta_{n}\right) \\
& =2\left(2\left\|x_{n}-x_{n+1}\right\|^{2}+\theta_{n}\right) .
\end{aligned}
$$

From (67) and $\lim _{n \rightarrow \infty} \theta_{n}=0$, we have

$$
\lim _{n \rightarrow \infty}\left\|x_{n}-y_{n}\right\|=0
$$

Since $y_{n}-x_{n}=\alpha_{n}\left(k_{n}-x_{n}\right)$ and $0<\alpha \leq \alpha_{n} \leq 1$, we have

$$
\alpha\left\|k_{n}-x_{n}\right\| \leq \alpha_{n}\left\|k_{n}-x_{n}\right\|=\left\|y_{n}-x_{n}\right\|,
$$

which immediately leads to

$$
\lim _{n \rightarrow \infty}\left\|k_{n}-x_{n}\right\|=0
$$

Also, utilizing Lemmas 9 and 23(b) we obtain from (46), (58), (59), and (62) that

$$
\begin{aligned}
& \left\|z_{n}-p\right\|^{2} \\
& =\| \beta_{n} x_{n}+\left(\left(1-\beta_{n}\right) I-s_{n} V\right) T_{n} G v_{n} \\
& +s_{n}\left[T_{n} x_{n}-\sigma_{n}\left(\mu F\left(T_{n} x_{n}\right)-\gamma Q x_{n}\right)\right]-p \|^{2} \\
& =\| \beta_{n}\left(x_{n}-p\right)+\left(1-\beta_{n}\right)\left(T_{n} G v_{n}-p\right) \\
& +s_{n}\left[T_{n} x_{n}-\sigma_{n}\left(\mu F\left(T_{n} x_{n}\right)-\gamma Q x_{n}\right)-V T_{n} G v_{n}\right] \|^{2} \\
& \leq\left\|\beta_{n}\left(x_{n}-p\right)+\left(1-\beta_{n}\right)\left(T_{n} G v_{n}-p\right)\right\|^{2} \\
& +2 s_{n}\left\langle T_{n} x_{n}-\sigma_{n}\left(\mu F\left(T_{n} x_{n}\right)-\gamma Q x_{n}\right)-V T_{n} G v_{n}, z_{n}-p\right\rangle \\
& =\beta_{n}\left\|x_{n}-p\right\|^{2}+\left(1-\beta_{n}\right)\left\|T_{n} G v_{n}-p\right\|^{2} \\
& -\beta_{n}\left(1-\beta_{n}\right)\left\|x_{n}-T_{n} G v_{n}\right\|^{2} \\
& +2 s_{n}\left\langle T_{n} x_{n}-\sigma_{n}\left(\mu F\left(T_{n} x_{n}\right)-\gamma Q x_{n}\right)-V T_{n} G v_{n}, z_{n}-p\right\rangle \\
& \leq \beta_{n}\left\|x_{n}-p\right\|^{2}+\left(1-\beta_{n}\right)\left\|G v_{n}-p\right\|^{2} \\
& -\beta_{n}\left(1-\beta_{n}\right)\left\|x_{n}-T_{n} G v_{n}\right\|^{2} \\
& +2 s_{n}\left\|T_{n} x_{n}-\sigma_{n}\left(\mu F\left(T_{n} x_{n}\right)-\gamma Q x_{n}\right)-V T_{n} G v_{n}\right\| \\
& \times\left\|z_{n}-p\right\| \\
& \leq \beta_{n}\left\|x_{n}-p\right\|^{2}+\left(1-\beta_{n}\right)\left\|v_{n}-p\right\|^{2} \\
& -\beta_{n}\left(1-\beta_{n}\right)\left\|x_{n}-T_{n} G v_{n}\right\|^{2} \\
& +2 s_{n}\left\|T_{n} x_{n}-\sigma_{n}\left(\mu F\left(T_{n} x_{n}\right)-\gamma Q x_{n}\right)-V T_{n} G v_{n}\right\| \\
& \times\left\|z_{n}-p\right\| \\
& \leq \beta_{n}\left\|x_{n}-p\right\|^{2}+\left(1-\beta_{n}\right)\left\|x_{n}-p\right\|^{2} \\
& -\beta_{n}\left(1-\beta_{n}\right)\left\|x_{n}-T_{n} G v_{n}\right\|^{2} \\
& +2 s_{n}\left\|T_{n} x_{n}-\sigma_{n}\left(\mu F\left(T_{n} x_{n}\right)-\gamma Q x_{n}\right)-V T_{n} G v_{n}\right\| \\
& \times\left\|z_{n}-p\right\| \\
& =\left\|x_{n}-p\right\|^{2}-\beta_{n}\left(1-\beta_{n}\right)\left\|x_{n}-T_{n} G v_{n}\right\|^{2} \\
& +2 s_{n}\left\|T_{n} x_{n}-\sigma_{n}\left(\mu F\left(T_{n} x_{n}\right)-\gamma Q x_{n}\right)-V T_{n} G v_{n}\right\| \\
& \times\left\|z_{n}-p\right\|
\end{aligned}
$$

and hence

$$
\begin{aligned}
\| y_{n} & -p \|^{2} \\
& \leq\left(1-\alpha_{n}\right)\left\|x_{n}-p\right\|^{2}+\alpha_{n}\left\|k_{n}-p\right\|^{2} \\
& \leq\left(1-\alpha_{n}\right)\left\|x_{n}-p\right\|^{2}+\alpha_{n}\left[\left(1+\gamma_{n}\right)\left\|z_{n}-p\right\|^{2}+c_{n}\right] \\
& \leq\left(1-\alpha_{n}\right)\left\|x_{n}-p\right\|^{2}+\alpha_{n}
\end{aligned}
$$




$$
\begin{aligned}
\times & {\left[\left(1+\gamma_{n}\right)\right.} \\
& \times\left(\left\|x_{n}-p\right\|^{2}-\beta_{n}\left(1-\beta_{n}\right)\left\|x_{n}-T_{n} G v_{n}\right\|^{2}\right. \\
& +2 s_{n}\left\|T_{n} x_{n}-\sigma_{n}\left(\mu F\left(T_{n} x_{n}\right)-\gamma Q x_{n}\right)-V T_{n} G v_{n}\right\| \\
& \left.\left.\quad \times\left\|z_{n}-p\right\|\right)+c_{n}\right] \\
\leq & \left(1-\alpha_{n}\right)\left\|x_{n}-p\right\|^{2}+\alpha_{n}\left(1+\gamma_{n}\right) \\
\times & \left(\left\|x_{n}-p\right\|^{2}-\beta_{n}\left(1-\beta_{n}\right)\left\|x_{n}-T_{n} G v_{n}\right\|^{2}\right. \\
& +2 s_{n}\left\|T_{n} x_{n}-\sigma_{n}\left(\mu F\left(T_{n} x_{n}\right)-\gamma Q x_{n}\right)-V T_{n} G v_{n}\right\| \\
& \left.\times\left\|z_{n}-p\right\|\right)+c_{n} \\
\leq & \left(1-\alpha_{n}\right)\left\|x_{n}-p\right\|^{2}+\alpha_{n}\left(1+\gamma_{n}\right)\left\|x_{n}-p\right\|^{2} \\
- & \alpha_{n}\left(1+\gamma_{n}\right) \beta_{n}\left(1-\beta_{n}\right)\left\|x_{n}-T_{n} G v_{n}\right\|^{2} \\
+ & \left(1+\gamma_{n}\right) 2 s_{n} \\
\times & \left\|T_{n} x_{n}-\sigma_{n}\left(\mu F\left(T_{n} x_{n}\right)-\gamma Q x_{n}\right)-V T_{n} G v_{n}\right\| \\
\times & \left\|z_{n}-p\right\|+c_{n} \\
\leq & \left(1+\gamma_{n}\right)\left\|x_{n}-p\right\|^{2}-\alpha_{n}\left(1+\gamma_{n}\right) \beta_{n}\left(1-\beta_{n}\right) \\
\times & \left\|x_{n}-T_{n} G v_{n}\right\|^{2} \\
+ & 2 s_{n}\left(1+\gamma_{n}\right) \\
\times & \left\|T_{n} x_{n}-\sigma_{n}\left(\mu F\left(T_{n} x_{n}\right)-\gamma Q x_{n}\right)-V T_{n} G v_{n}\right\| \\
\times & \left\|z_{n}-p\right\|+c_{n} .
\end{aligned}
$$

So, it follows that

$$
\begin{aligned}
\alpha(1+ & \left.\gamma_{n}\right) a(1-\widehat{a})\left\|x_{n}-T_{n} G v_{n}\right\|^{2} \\
\leq & \alpha_{n}\left(1+\gamma_{n}\right) \beta_{n}\left(1-\beta_{n}\right)\left\|x_{n}-T_{n} G v_{n}\right\|^{2} \\
\leq & \left\|x_{n}-p\right\|^{2}-\left\|y_{n}-p\right\|^{2}+\gamma_{n}\left\|x_{n}-p\right\|^{2} \\
& +2 s_{n}\left(1+\gamma_{n}\right) \\
& \times\left\|T_{n} x_{n}-\sigma_{n}\left(\mu F\left(T_{n} x_{n}\right)-\gamma Q x_{n}\right)-V T_{n} G v_{n}\right\| \\
& \times\left\|z_{n}-p\right\|+c_{n} \\
\leq & \left\|x_{n}-y_{n}\right\|\left(\left\|x_{n}-p\right\|+\left\|y_{n}-p\right\|\right)+\gamma_{n}\left\|x_{n}-p\right\|^{2} \\
& +2 s_{n}\left(1+\gamma_{n}\right) \\
& \times\left\|T_{n} x_{n}-\sigma_{n}\left(\mu F\left(T_{n} x_{n}\right)-\gamma Q x_{n}\right)-V T_{n} G v_{n}\right\| \\
& \times\left\|z_{n}-p\right\|+c_{n} .
\end{aligned}
$$

Since $\lim _{n \rightarrow \infty} s_{n}=0, \lim _{n \rightarrow \infty} \gamma_{n}=0$, and $\lim _{n \rightarrow \infty} c_{n}=0$, it follows from (69) and the boundedness of $\left\{x_{n}\right\},\left\{y_{n}\right\},\left\{z_{n}\right\}$, and $\left\{v_{n}\right\}$ that

$$
\lim _{n \rightarrow \infty}\left\|x_{n}-T_{n} G v_{n}\right\|=0 .
$$

Note that

$$
\begin{aligned}
\| z_{n}- & x_{n} \| \\
= & \|\left(1-\beta_{n}\right)\left(T_{n} G v_{n}-x_{n}\right) \\
& +s_{n}\left(T_{n} x_{n}-\sigma_{n}\left(\mu F\left(T_{n} x_{n}\right)-\gamma Q x_{n}\right)-V T_{n} G v_{n}\right) \| \\
\leq & \left(1-\beta_{n}\right)\left\|T_{n} G v_{n}-x_{n}\right\| \\
& +s_{n}\left\|T_{n} x_{n}-\sigma_{n}\left(\mu F\left(T_{n} x_{n}\right)-\gamma Q x_{n}\right)-V T_{n} G v_{n}\right\| \\
\leq & \left\|T_{n} G v_{n}-x_{n}\right\| \\
& +s_{n}\left\|T_{n} x_{n}-\sigma_{n}\left(\mu F\left(T_{n} x_{n}\right)-\gamma Q x_{n}\right)-V T_{n} G v_{n}\right\| .
\end{aligned}
$$

Hence, it follows from (75) and $\lim _{n \rightarrow \infty} s_{n}=0$ that

$$
\lim _{n \rightarrow \infty}\left\|x_{n}-z_{n}\right\|=0 \text {. }
$$

Note that

$$
\left\|k_{n}-z_{n}\right\| \leq\left\|k_{n}-x_{n}\right\|+\left\|x_{n}-z_{n}\right\| .
$$

Thus, we deduce from (71) and (77) that

$$
\lim _{n \rightarrow \infty}\left\|k_{n}-z_{n}\right\|=0 .
$$

Since $k_{n}-z_{n}=\left(1-\delta_{n}\right)\left(S^{n} z_{n}-z_{n}\right)$ and $k \leq \delta_{n} \leq d<1$, we have

$$
(1-d)\left\|S^{n} z_{n}-z_{n}\right\| \leq\left(1-\delta_{n}\right)\left\|S^{n} z_{n}-z_{n}\right\|=\left\|k_{n}-z_{n}\right\|,
$$

which, together with (79), yields

$$
\lim _{n \rightarrow \infty}\left\|S^{n} z_{n}-z_{n}\right\|=0
$$

Step 3. We prove that $\left\|x_{n}-u_{n}\right\| \rightarrow 0,\left\|x_{n}-v_{n}\right\| \rightarrow 0, \| v_{n}-$ $G v_{n}\|\rightarrow 0,\| v_{n}-P_{C}(I-(2 / L) \nabla f) v_{n} \| \rightarrow 0$, and $\left\|z_{n}-S z_{n}\right\| \rightarrow$ 0 as $n \rightarrow \infty$.

Indeed, from (57), (59), $\bar{\gamma}>1$, and $\gamma l \leq \tau$ it follows that

$$
\begin{aligned}
& \left\|z_{n}-p\right\|^{2} \\
& =\| \beta_{n}\left(x_{n}-p\right)+\left(\left(1-\beta_{n}\right) I-s_{n} V\right)\left(T_{n} G v_{n}-p\right) \\
& +s_{n}\left[\sigma_{n} \gamma\left(Q x_{n}-Q p\right)+\left(I-\sigma_{n} \mu F\right) T_{n} x_{n}\right. \\
& \left.\quad-\left(I-\sigma_{n} \mu F\right) T_{n} p\right]
\end{aligned}
$$




$$
\begin{aligned}
& +s_{n}\left[(I-V) p+\sigma_{n}(\gamma Q-\mu F) p\right] \|^{2} \\
& \leq \| \beta_{n}\left(x_{n}-p\right)+\left(\left(1-\beta_{n}\right) I-s_{n} V\right)\left(T_{n} G v_{n}-p\right) \\
& +s_{n}\left[\sigma_{n} \gamma\left(Q x_{n}-Q p\right)+\left(I-\sigma_{n} \mu F\right) T_{n} x_{n}\right. \\
& \left.-\left(I-\sigma_{n} \mu F\right) T_{n} p\right] \|^{2} \\
& +2 s_{n}\left\langle(I-V) p+\sigma_{n}(\gamma Q-\mu F) p, z_{n}-p\right\rangle \\
& \leq\left[\beta_{n}\left\|x_{n}-p\right\|+\left\|\left(1-\beta_{n}\right) I-s_{n} V\right\|\left\|T_{n} G v_{n}-p\right\|\right. \\
& +s_{n}\left(\sigma_{n} \gamma\left\|Q x_{n}-Q p\right\|\right. \\
& \left.\left.+\left\|\left(I-\sigma_{n} \mu F\right) T_{n} x_{n}-\left(I-\sigma_{n} \mu F\right) T_{n} p\right\|\right)\right]^{2} \\
& +2 s_{n}\left\langle(I-V) p+\sigma_{n}(\gamma Q-\mu F) p, z_{n}-p\right\rangle \\
& \leq\left[\beta_{n}\left\|x_{n}-p\right\|+\left(1-\beta_{n}-s_{n} \bar{\gamma}\right)\left\|G v_{n}-p\right\|\right. \\
& \left.+s_{n}\left(\sigma_{n} \gamma l\left\|x_{n}-p\right\|+\left(1-\sigma_{n} \tau\right)\left\|x_{n}-p\right\|\right)\right]^{2} \\
& +2 s_{n}\left\langle(I-V) p+\sigma_{n}(\gamma Q-\mu F) p, z_{n}-p\right\rangle \\
& =\left[\beta_{n}\left\|x_{n}-p\right\|+\left(1-\beta_{n}-s_{n} \bar{\gamma}\right)\left\|G v_{n}-p\right\|\right. \\
& \left.+s_{n}\left(1-\sigma_{n}(\tau-\gamma l)\right)\left\|x_{n}-p\right\|\right]^{2} \\
& +2 s_{n}\left\langle(I-V) p+\sigma_{n}(\gamma Q-\mu F) p, z_{n}-p\right\rangle \\
& \leq\left[\beta_{n}\left\|x_{n}-p\right\|+\left(1-\beta_{n}-s_{n} \bar{\gamma}\right)\left\|G v_{n}-p\right\|\right. \\
& \left.+s_{n}\left\|x_{n}-p\right\|\right]^{2} \\
& +2 s_{n}\left\langle(I-V) p+\sigma_{n}(\gamma Q-\mu F) p, z_{n}-p\right\rangle \\
& \leq\left[\left(\beta_{n}+s_{n} \bar{\gamma}\right)\left\|x_{n}-p\right\|+\left(1-\beta_{n}-s_{n} \bar{\gamma}\right)\left\|G v_{n}-p\right\|\right]^{2} \\
& +2 s_{n}\left\langle(I-V) p+\sigma_{n}(\gamma Q-\mu F) p, z_{n}-p\right\rangle \\
& \leq\left(\beta_{n}+s_{n} \bar{\gamma}\right)\left\|x_{n}-p\right\|^{2}+\left(1-\beta_{n}-s_{n} \bar{\gamma}\right)\left\|G v_{n}-p\right\|^{2} \\
& +2 s_{n}\left\langle(I-V) p+\sigma_{n}(\gamma Q-\mu F) p, z_{n}-p\right\rangle \\
& \leq\left(\beta_{n}+s_{n} \bar{\gamma}\right)\left\|x_{n}-p\right\|^{2}+\left(1-\beta_{n}-s_{n} \bar{\gamma}\right)\left\|v_{n}-p\right\|^{2} \\
& +2 s_{n}\left\langle(I-V) p+\sigma_{n}(\gamma Q-\mu F) p, z_{n}-p\right\rangle \\
& \leq\left(\beta_{n}+s_{n} \bar{\gamma}\right)\left\|x_{n}-p\right\|^{2}+\left(1-\beta_{n}-s_{n} \bar{\gamma}\right)\left\|u_{n}-p\right\|^{2} \\
& +2 s_{n}\left\langle(I-V) p+\sigma_{n}(\gamma Q-\mu F) p, z_{n}-p\right\rangle .
\end{aligned}
$$

Next let us show that

$$
\lim _{n \rightarrow \infty}\left\|x_{n}-u_{n}\right\|=0
$$

For $p \in \Omega$, we find from (46) that

$$
\begin{aligned}
& \left\|\Delta_{n}^{k} x_{n}-p\right\|^{2} \\
& =\left\|T_{r_{k, n}}^{\left(\Theta_{k}, \varphi_{k}\right)}\left(I-r_{k, n} B_{k}\right) \Delta_{n}^{k-1} x_{n}-T_{r_{k, n}}^{\left(\Theta_{k}, \varphi_{k}\right)}\left(I-r_{k, n} B_{k}\right) p\right\|^{2} \\
& =\left\|\left(I-r_{k, n} B_{k}\right) \Delta_{n}^{k-1} x_{n}-\left(I-r_{k, n} B_{k}\right) p\right\|^{2} \\
& \leq\left\|\Delta_{n}^{k-1} x_{n}-p\right\|^{2}+r_{k, n}\left(r_{k, n}-2 \mu_{k}\right)\left\|B_{k} \Delta_{n}^{k-1} x_{n}-B_{k} p\right\|^{2} \\
& \leq\left\|x_{n}-p\right\|^{2}+r_{k, n}\left(r_{k, n}-2 \mu_{k}\right)\left\|B_{k} \Delta_{n}^{k-1} x_{n}-B_{k} p\right\|^{2} \text {. }
\end{aligned}
$$

By (56), (82), and (84), we obtain

$$
\begin{aligned}
\| z_{n}- & p \|^{2} \\
\leq & \left(\beta_{n}+s_{n} \bar{\gamma}\right)\left\|x_{n}-p\right\|^{2}+\left(1-\beta_{n}-s_{n} \bar{\gamma}\right)\left\|u_{n}-p\right\|^{2} \\
& +2 s_{n}\left\langle(I-V) p+\sigma_{n}(\gamma Q-\mu F) p, z_{n}-p\right\rangle \\
\leq & \left(\beta_{n}+s_{n} \bar{\gamma}\right)\left\|x_{n}-p\right\|^{2}+\left(1-\beta_{n}-s_{n} \bar{\gamma}\right) \\
& \times\left\|\Delta_{n}^{k} x_{n}-p\right\|^{2} \\
& +2 s_{n}\left\langle(I-V) p+\sigma_{n}(\gamma Q-\mu F) p, z_{n}-p\right\rangle \\
\leq & \left(\beta_{n}+s_{n} \bar{\gamma}\right)\left\|x_{n}-p\right\|^{2}+\left(1-\beta_{n}-s_{n} \bar{\gamma}\right) \\
& \times\left[\left\|x_{n}-p\right\|^{2}+r_{k, n}\left(r_{k, n}-2 \mu_{k}\right)\left\|B_{k} \Delta_{n}^{k-1} x_{n}-B_{k} p\right\|^{2}\right] \\
& +2 s_{n}\left\langle(I-V) p+\sigma_{n}(\gamma Q-\mu F) p, z_{n}-p\right\rangle \\
= & \left\|x_{n}-p\right\|^{2}+\left(1-\beta_{n}-s_{n} \bar{\gamma}\right) r_{k, n}\left(r_{k, n}-2 \mu_{k}\right) \\
& \times\left\|B_{k} \Delta_{n}^{k-1} x_{n}-B_{k} p\right\|^{2} \\
& +2 s_{n}\left\langle(I-V) p+\sigma_{n}(\gamma Q-\mu F) p, z_{n}-p\right\rangle,
\end{aligned}
$$

which immediately yields

$$
\begin{aligned}
&\left(1-\widehat{a}-s_{n} \bar{\gamma}\right) r_{k, n}\left(2 \mu_{k}-r_{k, n}\right)\left\|B_{k} \Delta_{n}^{k-1} x_{n}-B_{k} p\right\|^{2} \\
& \leq\left(1-\beta_{n}-s_{n} \bar{\gamma}\right) r_{k, n}\left(2 \mu_{k}-r_{k, n}\right)\left\|B_{k} \Delta_{n}^{k-1} x_{n}-B_{k} p\right\|^{2} \\
& \leq\left\|x_{n}-p\right\|^{2}-\left\|z_{n}-p\right\|^{2} \\
&+2 s_{n}\left\langle(I-V) p+\sigma_{n}(\gamma Q-\mu F) p, z_{n}-p\right\rangle \\
& \leq\left\|x_{n}-z_{n}\right\|\left(\left\|x_{n}-p\right\|+\left\|z_{n}-p\right\|\right) \\
&+2 s_{n}(\|(I-V) p\|+\|(\gamma Q-\mu F) p\|)\left\|z_{n}-p\right\| .
\end{aligned}
$$

Since $\lim _{n \rightarrow \infty} s_{n}=0,\left\{r_{k, n}\right\} \subset\left[e_{k}, f_{k}\right] \subset\left(0,2 \mu_{k}\right)$, and $\left\{x_{n}\right\}$ and $\left\{z_{n}\right\}$ are bounded sequences, it follows from (77) that

$$
\lim _{n \rightarrow \infty}\left\|B_{k} \Delta_{n}^{k-1} x_{n}-B_{k} p\right\|=0, \quad k=1,2, \ldots, M .
$$


Journal of Applied Mathematics

13

By Proposition 8(iii) and (46), we have

$$
\begin{aligned}
& \left\|\Delta_{n}^{k} x_{n}-p\right\|^{2} \\
& =\left\|T_{r_{k, n}^{\left(\Theta_{k}, \varphi_{k}\right)}}\left(I-r_{k, n} B_{k}\right) \Delta_{n}^{k-1} x_{n}-T_{r_{k, n}}^{\left(\Theta_{k}, \varphi_{k}\right)}\left(I-r_{k, n} B_{k}\right) p\right\|^{2} \\
& \leq\left\langle\left(I-r_{k, n} B_{k}\right) \Delta_{n}^{k-1} x_{n}-\left(I-r_{k, n} B_{k}\right) p, \Delta_{n}^{k} x_{n}-p\right\rangle \\
& =\frac{1}{2}\left(\left\|\left(I-r_{k, n} B_{k}\right) \Delta_{n}^{k-1} x_{n}-\left(I-r_{k, n} B_{k}\right) p\right\|^{2}+\left\|\Delta_{n}^{k} x_{n}-p\right\|^{2}\right. \\
& \left.\quad-\left\|\left(I-r_{k, n} B_{k}\right) \Delta_{n}^{k-1} x_{n}-\left(I-r_{k, n} B_{k}\right) p-\left(\Delta_{n}^{k} x_{n}-p\right)\right\|^{2}\right) \\
& \leq \frac{1}{2}\left(\left\|\Delta_{n}^{k-1} x_{n}-p\right\|^{2}+\left\|\Delta_{n}^{k} x_{n}-p\right\|^{2}\right. \\
& \left.\quad-\left\|\Delta_{n}^{k-1} x_{n}-\Delta_{n}^{k} x_{n}-r_{k, n}\left(B_{k} \Delta_{n}^{k-1} x_{n}-B_{k} p\right)\right\|^{2}\right),
\end{aligned}
$$

which implies that

$$
\begin{aligned}
&\left\|\Delta_{n}^{k} x_{n}-p\right\|^{2} \\
& \leq\left\|\Delta_{n}^{k-1} x_{n}-p\right\|^{2} \\
& \quad-\left\|\Delta_{n}^{k-1} x_{n}-\Delta_{n}^{k} x_{n}-r_{k, n}\left(B_{k} \Delta_{n}^{k-1} x_{n}-B_{k} p\right)\right\|^{2} \\
&=\left\|\Delta_{n}^{k-1} x_{n}-p\right\|^{2}-\left\|\Delta_{n}^{k-1} x_{n}-\Delta_{n}^{k} x_{n}\right\|^{2} \\
& \quad-r_{k, n}^{2}\left\|B_{k} \Delta_{n}^{k-1} x_{n}-B_{k} p\right\|^{2} \\
& \quad+2 r_{k, n}\left\langle\Delta_{n}^{k-1} x_{n}-\Delta_{n}^{k} x_{n}, B_{k} \Delta_{n}^{k-1} x_{n}-B_{k} p\right\rangle \\
& \leq\left\|\Delta_{n}^{k-1} x_{n}-p\right\|^{2}-\left\|\Delta_{n}^{k-1} x_{n}-\Delta_{n}^{k} x_{n}\right\|^{2} \\
& \quad+2 r_{k, n}\left\|\Delta_{n}^{k-1} x_{n}-\Delta_{n}^{k} x_{n}\right\|\left\|B_{k} \Delta_{n}^{k-1} x_{n}-B_{k} p\right\| \\
& \leq\left\|x_{n}-p\right\|^{2}-\left\|\Delta_{n}^{k-1} x_{n}-\Delta_{n}^{k} x_{n}\right\|^{2} \\
&+2 r_{k, n}\left\|\Delta_{n}^{k-1} x_{n}-\Delta_{n}^{k} x_{n}\right\|\left\|B_{k} \Delta_{n}^{k-1} x_{n}-B_{k} p\right\| .
\end{aligned}
$$

From (82) and (89), we have

$$
\begin{aligned}
& \left\|z_{n}-p\right\|^{2} \\
& \leq\left(\beta_{n}+s_{n} \bar{\gamma}\right)\left\|x_{n}-p\right\|^{2}+\left(1-\beta_{n}-s_{n} \bar{\gamma}\right)\left\|u_{n}-p\right\|^{2} \\
& \quad+2 s_{n}\left\langle(I-V) p+\sigma_{n}(\gamma Q-\mu F) p, z_{n}-p\right\rangle \\
& \leq\left(\beta_{n}+s_{n} \bar{\gamma}\right)\left\|x_{n}-p\right\|^{2}+\left(1-\beta_{n}-s_{n} \bar{\gamma}\right)\left\|\Delta_{n}^{k} x_{n}-p\right\|^{2} \\
& \quad+2 s_{n}\left\langle(I-V) p+\sigma_{n}(\gamma Q-\mu F) p, z_{n}-p\right\rangle \\
& \leq\left(\beta_{n}+s_{n} \bar{\gamma}\right)\left\|x_{n}-p\right\|^{2}+\left(1-\beta_{n}-s_{n} \bar{\gamma}\right) \\
& \quad \times\left[\left\|x_{n}-p\right\|^{2}-\left\|\Delta_{n}^{k-1} x_{n}-\Delta_{n}^{k} x_{n}\right\|^{2}\right. \\
& \left.\quad+2 r_{k, n}\left\|\Delta_{n}^{k-1} x_{n}-\Delta_{n}^{k} x_{n}\right\|\left\|B_{k} \Delta_{n}^{k-1} x_{n}-B_{k} p\right\|\right]
\end{aligned}
$$

$$
\begin{aligned}
& +2 s_{n}\left\langle(I-V) p+\sigma_{n}(\gamma Q-\mu F) p, z_{n}-p\right\rangle \\
\leq & \left\|x_{n}-p\right\|^{2}-\left(1-\beta_{n}-s_{n} \bar{\gamma}\right)\left\|\Delta_{n}^{k-1} x_{n}-\Delta_{n}^{k} x_{n}\right\|^{2} \\
& +2 r_{k, n}\left\|\Delta_{n}^{k-1} x_{n}-\Delta_{n}^{k} x_{n}\right\|\left\|B_{k} \Delta_{n}^{k-1} x_{n}-B_{k} p\right\| \\
& +2 s_{n}\left\langle(I-V) p+\sigma_{n}(\gamma Q-\mu F) p, z_{n}-p\right\rangle,
\end{aligned}
$$

which leads to

$$
\begin{aligned}
&\left(1-\widehat{a}-s_{n} \bar{\gamma}\right)\left\|\Delta_{n}^{k-1} x_{n}-\Delta_{n}^{k} x_{n}\right\|^{2} \\
& \leq\left(1-\beta_{n}-s_{n} \bar{\gamma}\right)\left\|\Delta_{n}^{k-1} x_{n}-\Delta_{n}^{k} x_{n}\right\|^{2} \\
& \leq\left\|x_{n}-p\right\|^{2}-\left\|z_{n}-p\right\|^{2} \\
&+2 r_{k, n}\left\|\Delta_{n}^{k-1} x_{n}-\Delta_{n}^{k} x_{n}\right\|\left\|B_{k} \Delta_{n}^{k-1} x_{n}-B_{k} p\right\| \\
&+2 s_{n}\left\langle(I-V) p+\sigma_{n}(\gamma Q-\mu F) p, z_{n}-p\right\rangle \\
& \leq\left\|x_{n}-z_{n}\right\|\left(\left\|x_{n}-p\right\|+\left\|z_{n}-p\right\|\right) \\
&+2 r_{k, n}\left\|\Delta_{n}^{k-1} x_{n}-\Delta_{n}^{k} x_{n}\right\|\left\|B_{k} \Delta_{n}^{k-1} x_{n}-B_{k} p\right\| \\
&+2 s_{n}(\|(I-V) p\|+\|(\gamma Q-\mu F) p\|)\left\|z_{n}-p\right\| .
\end{aligned}
$$

Since $\lim _{n \rightarrow \infty} s_{n}=0,\left\{r_{k, n}\right\} \subset\left[e_{k}, f_{k}\right] \subset\left(0,2 \mu_{k}\right)$, and $\left\{x_{n}\right\}$ and $\left\{z_{n}\right\}$ are bounded sequences, it follows from (77) and (87) that

$$
\lim _{n \rightarrow \infty}\left\|\Delta_{n}^{k-1} x_{n}-\Delta_{n}^{k} x_{n}\right\|=0
$$

Hence we obtain from (92) that

$$
\begin{aligned}
\left\|x_{n}-u_{n}\right\|= & \left\|\Delta_{n}^{0} x_{n}-\Delta_{n}^{M} x_{n}\right\| \\
\leq & \left\|\Delta_{n}^{0} x_{n}-\Delta_{n}^{1} x_{n}\right\|+\left\|\Delta_{n}^{1} x_{n}-\Delta_{n}^{2} x_{n}\right\| \\
& +\cdots\left\|\Delta_{n}^{M-1} x_{n}-\Delta_{n}^{M} x_{n}\right\| \longrightarrow 0 \text { as } n \longrightarrow \infty .
\end{aligned}
$$

That is, (83) holds.

Next we show that $\lim _{n \rightarrow \infty}\left\|A_{i} \Lambda_{n}^{i} u_{n}-A_{i} p\right\|=0, i=$ $1,2, \ldots, N$. As a matter of fact, observe that

$$
\begin{aligned}
& \left\|\Lambda_{n}^{i} u_{n}-p\right\|^{2} \\
& \quad=\left\|P_{C}\left(I-\lambda_{i, n} A_{i}\right) \Lambda_{n}^{i-1} u_{n}-P_{C}\left(I-\lambda_{i, n} A_{i}\right) p\right\|^{2} \\
& \quad \leq\left\|\left(I-\lambda_{i, n} A_{i}\right) \Lambda_{n}^{i-1} u_{n}-\left(I-\lambda_{i, n} A_{i}\right) p\right\|^{2} \\
& \quad \leq\left\|\Lambda_{n}^{i-1} u_{n}-p\right\|^{2}+\lambda_{i, n}\left(\lambda_{i, n}-2 \eta_{i}\right)\left\|A_{i} \Lambda_{n}^{i-1} u_{n}-A_{i} p\right\|^{2} \\
& \quad \leq\left\|u_{n}-p\right\|^{2}+\lambda_{i, n}\left(\lambda_{i, n}-2 \eta_{i}\right)\left\|A_{i} \Lambda_{n}^{i-1} u_{n}-A_{i} p\right\|^{2} \\
& \quad \leq\left\|x_{n}-p\right\|^{2}+\lambda_{i, n}\left(\lambda_{i, n}-2 \eta_{i}\right)\left\|A_{i} \Lambda_{n}^{i-1} u_{n}-A_{i} p\right\|^{2} .
\end{aligned}
$$


Combining (57), (82), and (94), we have

$$
\begin{aligned}
&\left\|z_{n}-p\right\|^{2} \\
& \leq\left(\beta_{n}+s_{n} \bar{\gamma}\right)\left\|x_{n}-p\right\|^{2}+\left(1-\beta_{n}-s_{n} \bar{\gamma}\right)\left\|v_{n}-p\right\|^{2} \\
&+2 s_{n}\left\langle(I-V) p+\sigma_{n}(\gamma Q-\mu F) p, z_{n}-p\right\rangle \\
& \leq\left(\beta_{n}+s_{n} \bar{\gamma}\right)\left\|x_{n}-p\right\|^{2}+\left(1-\beta_{n}-s_{n} \bar{\gamma}\right)\left\|\Lambda_{n}^{i} u_{n}-p\right\|^{2} \\
&+2 s_{n}\left\langle(I-V) p+\sigma_{n}(\gamma Q-\mu F) p, z_{n}-p\right\rangle \\
& \leq\left(\beta_{n}+s_{n} \bar{\gamma}\right)\left\|x_{n}-p\right\|^{2}+\left(1-\beta_{n}-s_{n} \bar{\gamma}\right) \\
& \times\left[\left\|x_{n}-p\right\|^{2}+\lambda_{i, n}\left(\lambda_{i, n}-2 \eta_{i}\right)\left\|A_{i} \Lambda_{n}^{i-1} u_{n}-A_{i} p\right\|^{2}\right] \\
&+2 s_{n}(\|(I-V) p\|+\|(\gamma Q-\mu F) p\|)\left\|z_{n}-p\right\| \\
&=\left\|x_{n}-p\right\|^{2}+\left(1-\beta_{n}-s_{n} \bar{\gamma}\right) \lambda_{i, n}\left(\lambda_{i, n}-2 \eta_{i}\right) \\
& \times\left\|A_{i} \Lambda_{n}^{i-1} u_{n}-A_{i} p\right\|^{2} \\
&+2 s_{n}(\|(I-V) p\|+\|(\gamma Q-\mu F) p\|)\left\|z_{n}-p\right\|,
\end{aligned}
$$

which leads to

$$
\begin{aligned}
& \left(1-\widehat{a}-s_{n} \bar{\gamma}\right) \lambda_{i, n}\left(2 \eta_{i}-\lambda_{i, n}\right)\left\|A_{i} \Lambda_{n}^{i-1} u_{n}-A_{i} p\right\|^{2} \\
& \leq\left(1-\beta_{n}-s_{n} \bar{\gamma}\right) \lambda_{i, n}\left(2 \eta_{i}-\lambda_{i, n}\right)\left\|A_{i} \Lambda_{n}^{i-1} u_{n}-A_{i} p\right\|^{2} \\
& \leq\left\|x_{n}-p\right\|^{2}-\left\|z_{n}-p\right\|^{2} \\
& \quad+2 s_{n}(\|(I-V) p\|+\|(\gamma Q-\mu F) p\|)\left\|z_{n}-p\right\| \\
& \leq\left\|x_{n}-z_{n}\right\|\left(\left\|x_{n}-p\right\|+\left\|z_{n}-p\right\|\right) \\
& \quad+2 s_{n}(\|(I-V) p\|+\|(\gamma Q-\mu F) p\|)\left\|z_{n}-p\right\| .
\end{aligned}
$$

Since $\lim _{n \rightarrow \infty} s_{n}=0,\left\{\lambda_{i, n}\right\} \subset\left[a_{i}, b_{i}\right] \subset\left(0,2 \eta_{i}\right)$, and $\left\{x_{n}\right\}$ and $\left\{z_{n}\right\}$ are bounded sequences, it follows from (77) that

$$
\lim _{n \rightarrow \infty}\left\|A_{i} \Lambda_{n}^{i-1} u_{n}-A_{i} p\right\|=0, \quad i=1,2, \ldots, N .
$$

By Proposition 3(iii) and Lemma 23(a), we obtain

$$
\begin{aligned}
& \left\|\Lambda_{n}^{i} u_{n}-p\right\|^{2} \\
& =\left\|P_{C}\left(I-\lambda_{i, n} A_{i}\right) \Lambda_{n}^{i-1} u_{n}-P_{C}\left(I-\lambda_{i, n} A_{i}\right) p\right\|^{2} \\
& \leq\left\langle\left(I-\lambda_{i, n} A_{i}\right) \Lambda_{n}^{i-1} u_{n}-\left(I-\lambda_{i, n} A_{i}\right) p, \Lambda_{n}^{i} u_{n}-p\right\rangle \\
& =\frac{1}{2}\left(\left\|\left(I-\lambda_{i, n} A_{i}\right) \Lambda_{n}^{i-1} u_{n}-\left(I-\lambda_{i, n} A_{i}\right) p\right\|^{2}+\left\|\Lambda_{n}^{i} u_{n}-p\right\|^{2}\right. \\
& \quad-\|\left(I-\lambda_{i, n} A_{i}\right) \Lambda_{n}^{i-1} u_{n}-\left(I-\lambda_{i, n} A_{i}\right) p \\
& \left.\quad-\left(\Lambda_{n}^{i} u_{n}-p\right) \|^{2}\right)
\end{aligned}
$$

$$
\begin{aligned}
& \leq \frac{1}{2}\left(\left\|\Lambda_{n}^{i-1} u_{n}-p\right\|^{2}+\left\|\Lambda_{n}^{i} u_{n}-p\right\|^{2}\right. \\
& \left.\quad-\left\|\Lambda_{n}^{i-1} u_{n}-\Lambda_{n}^{i} u_{n}-\lambda_{i, n}\left(A_{i} \Lambda_{n}^{i-1} u_{n}-A_{i} p\right)\right\|^{2}\right) \\
& \leq \frac{1}{2}\left(\left\|u_{n}-p\right\|^{2}+\left\|\Lambda_{n}^{i} u_{n}-p\right\|^{2}\right. \\
& \left.\quad-\left\|\Lambda_{n}^{i-1} u_{n}-\Lambda_{n}^{i} u_{n}-\lambda_{i, n}\left(A_{i} \Lambda_{n}^{i-1} u_{n}-A_{i} p\right)\right\|^{2}\right) \\
& \leq \frac{1}{2}\left(\left\|x_{n}-p\right\|^{2}+\left\|\Lambda_{n}^{i} u_{n}-p\right\|^{2}\right. \\
& \left.\quad-\left\|\Lambda_{n}^{i-1} u_{n}-\Lambda_{n}^{i} u_{n}-\lambda_{i, n}\left(A_{i} \Lambda_{n}^{i-1} u_{n}-A_{i} p\right)\right\|^{2}\right),
\end{aligned}
$$

which implies

$$
\begin{aligned}
& \left\|\Lambda_{n}^{i} u_{n}-p\right\|^{2} \\
& \leq\left\|x_{n}-p\right\|^{2}-\left\|\Lambda_{n}^{i-1} u_{n}-\Lambda_{n}^{i} u_{n}-\lambda_{i, n}\left(A_{i} \Lambda_{n}^{i-1} u_{n}-A_{i} p\right)\right\|^{2} \\
& =\left\|x_{n}-p\right\|^{2}-\left\|\Lambda_{n}^{i-1} u_{n}-\Lambda_{n}^{i} u_{n}\right\|^{2} \\
& -\lambda_{i, n}^{2}\left\|A_{i} \Lambda_{n}^{i-1} u_{n}-A_{i} p\right\|^{2} \\
& +2 \lambda_{i, n}\left\langle\Lambda_{n}^{i-1} u_{n}-\Lambda_{n}^{i} u_{n}, A_{i} \Lambda_{n}^{i-1} u_{n}-A_{i} p\right\rangle \\
& \leq\left\|x_{n}-p\right\|^{2}-\left\|\Lambda_{n}^{i-1} u_{n}-\Lambda_{n}^{i} u_{n}\right\|^{2} \\
& +2 \lambda_{i, n}\left\|\Lambda_{n}^{i-1} u_{n}-\Lambda_{n}^{i} u_{n}\right\|\left\|A_{i} \Lambda_{n}^{i-1} u_{n}-A_{i} p\right\| .
\end{aligned}
$$

Combining (57), (82), and (99), we have

$\left\|z_{n}-p\right\|^{2}$

$$
\begin{aligned}
\leq & \left(\beta_{n}+s_{n} \bar{\gamma}\right)\left\|x_{n}-p\right\|^{2}+\left(1-\beta_{n}-s_{n} \bar{\gamma}\right)\left\|v_{n}-p\right\|^{2} \\
& +2 s_{n}\left\langle(I-V) p+\sigma_{n}(\gamma Q-\mu F) p, z_{n}-p\right\rangle \\
\leq & \left(\beta_{n}+s_{n} \bar{\gamma}\right)\left\|x_{n}-p\right\|^{2}+\left(1-\beta_{n}-s_{n} \bar{\gamma}\right)\left\|\Lambda_{n}^{i} u_{n}-p\right\|^{2} \\
& +2 s_{n}\left\langle(I-V) p+\sigma_{n}(\gamma Q-\mu F) p, z_{n}-p\right\rangle \\
\leq & \left(\beta_{n}+s_{n} \bar{\gamma}\right)\left\|x_{n}-p\right\|^{2}+\left(1-\beta_{n}-s_{n} \bar{\gamma}\right) \\
& \times\left[\left\|x_{n}-p\right\|^{2}-\left\|\Lambda_{n}^{i-1} u_{n}-\Lambda_{n}^{i} u_{n}\right\|^{2}\right. \\
& \left.+2 \lambda_{i, n}\left\|\Lambda_{n}^{i-1} u_{n}-\Lambda_{n}^{i} u_{n}\right\|\left\|A_{i} \Lambda_{n}^{i-1} u_{n}-A_{i} p\right\|\right] \\
& +2 s_{n}\left\langle(I-V) p+\sigma_{n}(\gamma Q-\mu F) p, z_{n}-p\right\rangle \\
\leq & \left\|x_{n}-p\right\|^{2}-\left(1-\beta_{n}-s_{n} \bar{\gamma}\right)\left\|\Lambda_{n}^{i-1} u_{n}-\Lambda_{n}^{i} u_{n}\right\|^{2} \\
& +2 \lambda_{i, n}\left\|\Lambda_{n}^{i-1} u_{n}-\Lambda_{n}^{i} u_{n}\right\|\left\|A_{i} \Lambda_{n}^{i-1} u_{n}-A_{i} p\right\| \\
& +2 s_{n}\left\langle(I-V) p+\sigma_{n}(\gamma Q-\mu F) p, z_{n}-p\right\rangle
\end{aligned}
$$


which yields

$$
\begin{aligned}
&\left(1-\widehat{a}-s_{n} \bar{\gamma}\right)\left\|\Lambda_{n}^{i-1} u_{n}-\Lambda_{n}^{i} u_{n}\right\|^{2} \\
& \leq\left(1-\beta_{n}-s_{n} \bar{\gamma}\right)\left\|\Lambda_{n}^{i-1} u_{n}-\Lambda_{n}^{i} u_{n}\right\|^{2} \\
& \leq\left\|x_{n}-p\right\|^{2}-\left\|z_{n}-p\right\|^{2} \\
&+2 \lambda_{i, n}\left\|\Lambda_{n}^{i-1} u_{n}-\Lambda_{n}^{i} u_{n}\right\|\left\|A_{i} \Lambda_{n}^{i-1} u_{n}-A_{i} p\right\| \\
&+2 s_{n}\left\langle(I-V) p+\sigma_{n}(\gamma Q-\mu F) p, z_{n}-p\right\rangle \\
& \leq\left\|x_{n}-z_{n}\right\|\left(\left\|x_{n}-p\right\|+\left\|z_{n}-p\right\|\right) \\
&+2 \lambda_{i, n}\left\|\Lambda_{n}^{i-1} u_{n}-\Lambda_{n}^{i} u_{n}\right\|\left\|A_{i} \Lambda_{n}^{i-1} u_{n}-A_{i} p\right\| \\
&+2 s_{n}(\|(I-V) p\|+\|(\gamma Q-\mu F) p\|)\left\|z_{n}-p\right\| .
\end{aligned}
$$

Since $\lim _{n \rightarrow \infty} s_{n}=0$ and $\left\{x_{n}\right\},\left\{z_{n}\right\}$, and $\left\{u_{n}\right\}$ are bounded, from (77) and (97) we get

$$
\lim _{n \rightarrow \infty}\left\|\Lambda_{n}^{i-1} u_{n}-\Lambda_{n}^{i} u_{n}\right\|=0 .
$$

From (102) we get

$$
\begin{aligned}
\left\|u_{n}-v_{n}\right\|= & \left\|\Lambda_{n}^{0} u_{n}-\Lambda_{n}^{N} u_{n}\right\| \\
\leq & \left\|\Lambda_{n}^{0} u_{n}-\Lambda_{n}^{1} u_{n}\right\|+\left\|\Lambda_{n}^{1} u_{n}-\Lambda_{n}^{2} u_{n}\right\| \\
& +\cdots+\left\|\Lambda_{n}^{N-1} u_{n}-\Lambda_{n}^{N} u_{n}\right\| \longrightarrow 0 \\
& \text { as } n \longrightarrow \infty .
\end{aligned}
$$

Taking into account that $\left\|x_{n}-v_{n}\right\| \leq\left\|x_{n}-u_{n}\right\|+\left\|u_{n}-v_{n}\right\|$, we conclude from (83) and (103) that

$$
\lim _{n \rightarrow \infty}\left\|x_{n}-v_{n}\right\|=0
$$

On the other hand, for simplicity, we write $\widetilde{p}=P_{C}(I-$ $\left.v_{2} F_{2}\right) p, \widetilde{v}_{n}=P_{C}\left(I-v_{2} F_{2}\right) v_{n}$, and $w_{n}=G v_{n}=P_{C}\left(I-v_{1} F_{1}\right) \widetilde{v}_{n}$ for all $n \geq 1$. Then

$$
p=G p=P_{C}\left(I-v_{1} F_{1}\right) \tilde{p}=P_{C}\left(I-v_{1} F_{1}\right) P_{C}\left(I-v_{2} F_{2}\right) p .
$$

We now show that $\lim _{n \rightarrow \infty}\left\|G v_{n}-v_{n}\right\|=0$; that is, $\lim _{n \rightarrow \infty}\left\|w_{n}-v_{n}\right\|=0$. As a matter of fact, for $p \in \Omega$, it follows from (58), (59), and (82) that

$$
\begin{aligned}
& \left\|z_{n}-p\right\|^{2} \\
& \quad \leq\left(\beta_{n}+s_{n} \bar{\gamma}\right)\left\|x_{n}-p\right\|^{2}+\left(1-\beta_{n}-s_{n} \bar{\gamma}\right)
\end{aligned}
$$

$$
\begin{aligned}
& \times\left\|G v_{n}-p\right\|^{2} \\
& +2 s_{n}\left\langle(I-V) p+\sigma_{n}(\gamma Q-\mu F) p, z_{n}-p\right\rangle \\
= & \left(\beta_{n}+s_{n} \bar{\gamma}\right)\left\|x_{n}-p\right\|^{2}+\left(1-\beta_{n}-s_{n} \bar{\gamma}\right) \\
& \times\left\|w_{n}-p\right\|^{2} \\
& +2 s_{n}\left\langle(I-V) p+\sigma_{n}(\gamma Q-\mu F) p, z_{n}-p\right\rangle \\
\leq & \left(\beta_{n}+s_{n} \bar{\gamma}\right)\left\|x_{n}-p\right\|^{2}+\left(1-\beta_{n}-s_{n} \bar{\gamma}\right) \\
& \times\left[\left\|\widetilde{v}_{n}-\tilde{p}\right\|^{2}+v_{1}\left(v_{1}-2 \zeta_{1}\right)\left\|F_{1} \widetilde{v}_{n}-F_{1} \tilde{p}\right\|^{2}\right] \\
& +2 s_{n}\left\langle(I-V) p+\sigma_{n}(\gamma Q-\mu F) p, z_{n}-p\right\rangle \\
\leq & \left(\beta_{n}+s_{n} \bar{\gamma}\right)\left\|x_{n}-p\right\|^{2}+\left(1-\beta_{n}-s_{n} \bar{\gamma}\right) \\
\times & {\left[\left\|v_{n}-p\right\|^{2}+v_{2}\left(v_{2}-2 \zeta_{2}\right)\left\|F_{2} v_{n}-F_{2} p\right\|^{2}\right.} \\
& \left.+v_{1}\left(v_{1}-2 \zeta_{1}\right)\left\|F_{1} \widetilde{v}_{n}-F_{1} \tilde{p}\right\|^{2}\right] \\
& +2 s_{n}(\|(I-V) p\|+\|(\gamma Q-\mu F) p\|)\left\|z_{n}-p\right\| \\
\leq & \left(\beta_{n}+s_{n} \bar{\gamma}\right)\left\|x_{n}-p\right\|^{2}+\left(1-\beta_{n}-s_{n} \bar{\gamma}\right) \\
& \times\left[\left\|x_{n}-p\right\|^{2}+v_{2}\left(v_{2}-2 \zeta_{2}\right)\left\|F_{2} v_{n}-F_{2} p\right\|^{2}\right. \\
& \left.+v_{1}\left(v_{1}-2 \zeta_{1}\right)\left\|F_{1} \widetilde{v}_{n}-F_{1} \tilde{p}\right\|^{2}\right] \\
+ & +2 s_{n}(\|(I-V) p\|+\|(\gamma Q-\mu F) p\|)\left\|z_{n}-p\right\| \\
= & +x_{n}-p \|^{2}+\left(1-\beta_{n}-s_{n} \bar{\gamma}\right) \\
\times & {\left[v_{2}\left(v_{2}-2 \zeta_{2}\right)\left\|F_{2} v_{n}-F_{2} p\right\|^{2}\right.} \\
+ & \left.\left.2 s_{n}\right)\left\|F_{1} \widetilde{v}_{n}-F_{1} \tilde{p}\right\|^{2}\right] \\
&
\end{aligned}
$$

which immediately yields

$$
\begin{gathered}
\left(1-\widehat{a}-s_{n} \bar{\gamma}\right)\left[v_{2}\left(2 \zeta_{2}-v_{2}\right)\left\|F_{2} v_{n}-F_{2} p\right\|^{2}\right. \\
\left.+v_{1}\left(2 \zeta_{1}-v_{1}\right)\left\|F_{1} \widetilde{v}_{n}-F_{1} \tilde{p}\right\|^{2}\right] \\
\leq\left(1-\beta_{n}-s_{n} \bar{\gamma}\right)\left[v_{2}\left(2 \zeta_{2}-v_{2}\right)\left\|F_{2} v_{n}-F_{2} p\right\|^{2}\right. \\
\left.+v_{1}\left(2 \zeta_{1}-v_{1}\right)\left\|F_{1} \widetilde{v}_{n}-F_{1} \tilde{p}\right\|^{2}\right] \\
\leq\left\|x_{n}-p\right\|^{2}-\left\|z_{n}-p\right\|^{2} \\
+2 s_{n}(\|(I-V) p\|+\|(\gamma Q-\mu F) p\|)\left\|z_{n}-p\right\| \\
\leq\left\|x_{n}-z_{n}\right\|\left(\left\|x_{n}-p\right\|+\left\|z_{n}-p\right\|\right) \\
+2 s_{n}(\|(I-V) p\|+\|(\gamma Q-\mu F) p\|)\left\|z_{n}-p\right\| .
\end{gathered}
$$


Since $\lim _{n \rightarrow \infty} s_{n}=0$ and $\left\{x_{n}\right\}$ and $\left\{z_{n}\right\}$ are bounded, from (77) we get

$$
\lim _{n \rightarrow \infty}\left\|F_{2} v_{n}-F_{2} p\right\|=0, \quad \lim _{n \rightarrow \infty}\left\|F_{1} \widetilde{v}_{n}-F_{1} \tilde{p}\right\|=0 .
$$

Also, in terms of the firm nonexpansivity of $P_{C}$ and the $\zeta_{j^{-}}$ inverse strong monotonicity of $F_{j}$ for $j=1,2$, we obtain from $v_{j} \in\left(0,2 \zeta_{j}\right), j=1,2$, and (59) that

$$
\begin{aligned}
& \left\|\widetilde{v}_{n}-\widetilde{p}\right\|^{2} \\
& =\left\|P_{C}\left(I-v_{2} F_{2}\right) v_{n}-P_{C}\left(I-v_{2} F_{2}\right) p\right\|^{2} \\
& \leq\left\langle\left(I-v_{2} F_{2}\right) v_{n}-\left(I-v_{2} F_{2}\right) p, \widetilde{v}_{n}-\tilde{p}\right\rangle \\
& =\frac{1}{2}\left[\left\|\left(I-v_{2} F_{2}\right) v_{n}-\left(I-v_{2} F_{2}\right) p\right\|^{2}+\left\|\widetilde{v}_{n}-\widetilde{p}\right\|^{2}\right. \\
& \left.-\left\|\left(I-v_{2} F_{2}\right) v_{n}-\left(I-v_{2} F_{2}\right) p-\left(\widetilde{v}_{n}-\tilde{p}\right)\right\|^{2}\right] \\
& \leq \frac{1}{2}\left[\left\|v_{n}-p\right\|^{2}+\left\|\widetilde{v}_{n}-\widetilde{p}\right\|^{2}\right. \\
& \left.-\left\|\left(v_{n}-\widetilde{v}_{n}\right)-v_{2}\left(F_{2} v_{n}-F_{2} p\right)-(p-\tilde{p})\right\|^{2}\right] \\
& =\frac{1}{2}\left[\left\|v_{n}-p\right\|^{2}+\left\|\widetilde{v}_{n}-\widetilde{p}\right\|^{2}\right. \\
& -\left\|\left(v_{n}-\widetilde{v}_{n}\right)-(p-\tilde{p})\right\|^{2} \\
& +2 v_{2}\left\langle\left(v_{n}-\widetilde{v}_{n}\right)-(p-\widetilde{p}), F_{2} v_{n}-F_{2} p\right\rangle \\
& \left.-v_{2}^{2}\left\|F_{2} v_{n}-F_{2} p\right\|^{2}\right] \text {, } \\
& \left\|w_{n}-p\right\|^{2} \\
& =\left\|P_{C}\left(I-v_{1} F_{1}\right) \widetilde{v}_{n}-P_{C}\left(I-v_{1} F_{1}\right) \tilde{p}\right\|^{2} \\
& \leq\left\langle\left(I-v_{1} F_{1}\right) \widetilde{v}_{n}-\left(I-v_{1} F_{1}\right) \tilde{p}, w_{n}-p\right\rangle \\
& =\frac{1}{2}\left[\left\|\left(I-v_{1} F_{1}\right) \widetilde{v}_{n}-\left(I-v_{1} F_{1}\right) \tilde{p}\right\|^{2}+\left\|w_{n}-p\right\|^{2}\right. \\
& \left.-\left\|\left(I-v_{1} F_{1}\right) \widetilde{v}_{n}-\left(I-v_{1} F_{1}\right) \tilde{p}-\left(w_{n}-p\right)\right\|^{2}\right] \\
& \leq \frac{1}{2}\left[\left\|\widetilde{v}_{n}-\tilde{p}\right\|^{2}+\left\|w_{n}-p\right\|^{2}\right. \\
& -\left\|\left(\widetilde{v}_{n}-w_{n}\right)+(p-\widetilde{p})\right\|^{2} \\
& +2 v_{1}\left\langle F_{1} \widetilde{v}_{n}-F_{1} \tilde{p},\left(\widetilde{v}_{n}-w_{n}\right)+(p-\widetilde{p})\right\rangle \\
& \left.-v_{1}^{2}\left\|F_{1} \widetilde{v}_{n}-F_{1} \tilde{p}\right\|^{2}\right] \\
& \leq \frac{1}{2}\left[\left\|v_{n}-p\right\|^{2}+\left\|w_{n}-p\right\|^{2}\right. \\
& -\left\|\left(\widetilde{v}_{n}-w_{n}\right)+(p-\tilde{p})\right\|^{2} \\
& \left.+2 v_{1}\left\langle F_{1} \widetilde{v}_{n}-F_{1} \tilde{p},\left(\widetilde{v}_{n}-w_{n}\right)+(p-\tilde{p})\right\rangle\right] \text {. }
\end{aligned}
$$

Thus, we have

$$
\begin{aligned}
\left\|\widetilde{v}_{n}-\tilde{p}\right\|^{2} & \\
\leq & \left\|v_{n}-p\right\|^{2}-\left\|\left(v_{n}-\widetilde{v}_{n}\right)-(p-\tilde{p})\right\|^{2} \\
& +2 v_{2}\left\langle\left(v_{n}-\widetilde{v}_{n}\right)-(p-\tilde{p}), F_{2} v_{n}-F_{2} p\right\rangle \\
& \quad-v_{2}^{2}\left\|F_{2} v_{n}-F_{2} p\right\|^{2}, \\
\left\|w_{n}-p\right\|^{2} & \\
\leq & \left\|v_{n}-p\right\|^{2}-\left\|\left(\widetilde{v}_{n}-w_{n}\right)+(p-\tilde{p})\right\|^{2} \\
& +2 v_{1}\left\|F_{1} \widetilde{v}_{n}-F_{1} \tilde{p}\right\|\left\|\left(\widetilde{v}_{n}-w_{n}\right)+(p-\tilde{p})\right\| .
\end{aligned}
$$

Consequently, from (58), (106), and (110) it follows that

$$
\begin{aligned}
& \left\|z_{n}-p\right\|^{2} \\
& \leq\left(\beta_{n}+s_{n} \bar{\gamma}\right)\left\|x_{n}-p\right\|^{2}+\left(1-\beta_{n}-s_{n} \bar{\gamma}\right) \\
& \times\left[\left\|\widetilde{v}_{n}-\widetilde{p}\right\|^{2}+\nu_{1}\left(\nu_{1}-2 \zeta_{1}\right)\left\|F_{1} \widetilde{v}_{n}-F_{1} \tilde{p}\right\|^{2}\right] \\
& +2 s_{n}\left\langle(I-V) p+\sigma_{n}(\gamma Q-\mu F) p, z_{n}-p\right\rangle \\
& \leq\left(\beta_{n}+s_{n} \bar{\gamma}\right)\left\|x_{n}-p\right\|^{2}+\left(1-\beta_{n}-s_{n} \bar{\gamma}\right)\left\|\widetilde{v}_{n}-\tilde{p}\right\|^{2} \\
& +2 s_{n}(\|(I-V) p\|+\|(\gamma Q-\mu F) p\|)\left\|z_{n}-p\right\| \\
& \leq\left(\beta_{n}+s_{n} \bar{\gamma}\right)\left\|x_{n}-p\right\|^{2}+\left(1-\beta_{n}-s_{n} \bar{\gamma}\right) \\
& \times\left[\left\|v_{n}-p\right\|^{2}-\left\|\left(v_{n}-\widetilde{v}_{n}\right)-(p-\tilde{p})\right\|^{2}\right. \\
& +2 v_{2}\left\langle\left(v_{n}-\widetilde{v}_{n}\right)-(p-\widetilde{p}), F_{2} v_{n}-F_{2} p\right\rangle \\
& \left.-v_{2}^{2}\left\|F_{2} v_{n}-F_{2} p\right\|^{2}\right] \\
& +2 s_{n}(\|(I-V) p\|+\|(\gamma Q-\mu F) p\|)\left\|z_{n}-p\right\| \\
& \leq\left(\beta_{n}+s_{n} \bar{\gamma}\right)\left\|x_{n}-p\right\|^{2}+\left(1-\beta_{n}-s_{n} \bar{\gamma}\right) \\
& \times\left[\left\|x_{n}-p\right\|^{2}-\left\|\left(v_{n}-\widetilde{v}_{n}\right)-(p-\tilde{p})\right\|^{2}\right. \\
& \left.+2 v_{2}\left\|\left(v_{n}-\widetilde{v}_{n}\right)-(p-\widetilde{p})\right\|\left\|F_{2} v_{n}-F_{2} p\right\|\right] \\
& +2 s_{n}(\|(I-V) p\|+\|(\gamma Q-\mu F) p\|)\left\|z_{n}-p\right\| \\
& \leq\left\|x_{n}-p\right\|^{2}-\left(1-\beta_{n}-s_{n} \bar{\gamma}\right)\left\|\left(v_{n}-\widetilde{v}_{n}\right)-(p-\tilde{p})\right\|^{2} \\
& +2 v_{2}\left\|\left(v_{n}-\widetilde{v}_{n}\right)-(p-\tilde{p})\right\|\left\|F_{2} v_{n}-F_{2} p\right\| \\
& +2 s_{n}(\|(I-V) p\|+\|(\gamma Q-\mu F) p\|)\left\|z_{n}-p\right\|,
\end{aligned}
$$


which hence leads to

$$
\begin{aligned}
&\left(1-\widehat{a}-s_{n} \bar{\gamma}\right)\left\|\left(v_{n}-\widetilde{v}_{n}\right)-(p-\widetilde{p})\right\|^{2} \\
& \leq\left(1-\beta_{n}-s_{n} \bar{\gamma}\right)\left\|\left(v_{n}-\widetilde{v}_{n}\right)-(p-\widetilde{p})\right\|^{2} \\
& \leq\left\|x_{n}-p\right\|^{2}-\left\|z_{n}-p\right\|^{2} \\
&+2 v_{2}\left\|\left(v_{n}-\widetilde{v}_{n}\right)-(p-\widetilde{p})\right\|\left\|F_{2} v_{n}-F_{2} p\right\| \\
&+2 s_{n}(\|(I-V) p\|+\|(\gamma Q-\mu F) p\|)\left\|z_{n}-p\right\| \\
& \leq\left\|x_{n}-z_{n}\right\|\left(\left\|x_{n}-p\right\|+\left\|z_{n}-p\right\|\right) \\
&+2 v_{2}\left\|\left(v_{n}-\widetilde{v}_{n}\right)-(p-\widetilde{p})\right\|\left\|F_{2} v_{n}-F_{2} p\right\| \\
&+2 s_{n}(\|(I-V) p\|+\|(\gamma Q-\mu F) p\|)\left\|z_{n}-p\right\| .
\end{aligned}
$$

Since $\lim _{n \rightarrow \infty} s_{n}=0$ and $\left\{x_{n}\right\},\left\{z_{n}\right\},\left\{v_{n}\right\}$, and $\left\{\widetilde{v}_{n}\right\}$ are bounded sequences, we conclude from (77) and (108) that

$$
\lim _{n \rightarrow \infty}\left\|\left(v_{n}-\tilde{v}_{n}\right)-(p-\tilde{p})\right\|=0 .
$$

Furthermore, from (58), (106), and (111) it follows that

$$
\begin{aligned}
\| z_{n}- & p \|^{2} \\
\leq & \left(\beta_{n}+s_{n} \bar{\gamma}\right)\left\|x_{n}-p\right\|^{2}+\left(1-\beta_{n}-s_{n} \bar{\gamma}\right) \\
& \times\left\|w_{n}-p\right\|^{2} \\
& +2 s_{n}\left\langle(I-V) p+\sigma_{n}(\gamma Q-\mu F) p, z_{n}-p\right\rangle \\
\leq & \left(\beta_{n}+s_{n} \bar{\gamma}\right)\left\|x_{n}-p\right\|^{2}+\left(1-\beta_{n}-s_{n} \bar{\gamma}\right) \\
& \times\left[\left\|v_{n}-p\right\|^{2}-\left\|\left(\widetilde{v}_{n}-w_{n}\right)+(p-\tilde{p})\right\|^{2}\right. \\
& \left.+2 v_{1}\left\|F_{1} \widetilde{v}_{n}-F_{1} \tilde{p}\right\|\left\|\left(\widetilde{v}_{n}-w_{n}\right)+(p-\tilde{p})\right\|\right] \\
& +2 s_{n}(\|(I-V) p\|+\|(\gamma Q-\mu F) p\|)\left\|z_{n}-p\right\| \\
\leq & \left(\beta_{n}+s_{n} \bar{\gamma}\right)\left\|x_{n}-p\right\|^{2}+\left(1-\beta_{n}-s_{n} \bar{\gamma}\right) \\
& \times\left[\left\|x_{n}-p\right\|^{2}-\left\|\left(\widetilde{v}_{n}-w_{n}\right)+(p-\tilde{p})\right\|^{2}\right. \\
& \left.+2 v_{1}\left\|F_{1} \widetilde{v}_{n}-F_{1} \tilde{p}\right\|\left\|\left(\widetilde{v}_{n}-w_{n}\right)+(p-\tilde{p})\right\|\right] \\
& +2 s_{n}(\|(I-V) p\|+\|(\gamma Q-\mu F) p\|)\left\|z_{n}-p\right\| \\
\leq & \left\|x_{n}-p\right\|^{2}-\left(1-\beta_{n}-s_{n} \bar{\gamma}\right) \\
\times & \left\|\left(\widetilde{v}_{n}-w_{n}\right)+(p-\widetilde{p})\right\|^{2} \\
+ & 2 v_{1}\left\|F_{1} \widetilde{v}_{n}-F_{1} \tilde{p}\right\|\left\|\left(\widetilde{v}_{n}-w_{n}\right)+(p-\tilde{p})\right\| \\
+ & 2 s_{n}(\|(I-V) p\|+\|(\gamma Q-\mu F) p\|)\left\|z_{n}-p\right\|,
\end{aligned}
$$

which hence yields

$$
\begin{aligned}
&\left(1-\widehat{a}-s_{n} \bar{\gamma}\right)\left\|\left(\widetilde{v}_{n}-w_{n}\right)+(p-\widetilde{p})\right\|^{2} \\
& \leq\left(1-\beta_{n}-s_{n} \bar{\gamma}\right)\left\|\left(\widetilde{v}_{n}-w_{n}\right)+(p-\widetilde{p})\right\|^{2} \\
& \leq\left\|x_{n}-p\right\|^{2}-\left\|z_{n}-p\right\|^{2} \\
&+2 v_{1}\left\|F_{1} \widetilde{v}_{n}-F_{1} \tilde{p}\right\|\left\|\left(\widetilde{v}_{n}-w_{n}\right)+(p-\widetilde{p})\right\| \\
&+2 s_{n}(\|(I-V) p\|+\|(\gamma Q-\mu F) p\|)\left\|z_{n}-p\right\| \\
& \leq\left\|x_{n}-z_{n}\right\|\left(\left\|x_{n}-p\right\|+\left\|z_{n}-p\right\|\right) \\
&+2 v_{1}\left\|F_{1} \widetilde{v}_{n}-F_{1} \tilde{p}\right\|\left\|\left(\widetilde{v}_{n}-w_{n}\right)+(p-\tilde{p})\right\| \\
&+2 s_{n}(\|(I-V) p\|+\|(\gamma Q-\mu F) p\|)\left\|z_{n}-p\right\| .
\end{aligned}
$$

Since $\lim _{n \rightarrow \infty} s_{n}=0$ and $\left\{x_{n}\right\},\left\{z_{n}\right\},\left\{w_{n}\right\}$, and $\left\{\widetilde{v}_{n}\right\}$ are bounded sequences, we conclude from (77) and (108) that

$$
\lim _{n \rightarrow \infty}\left\|\left(\widetilde{v}_{n}-w_{n}\right)+(p-\widetilde{p})\right\|=0 .
$$

Note that

$$
\begin{aligned}
& \left\|v_{n}-w_{n}\right\| \\
& \quad \leq\left\|\left(v_{n}-\widetilde{v}_{n}\right)-(p-\tilde{p})\right\|+\left\|\left(\widetilde{v}_{n}-w_{n}\right)+(p-\widetilde{p})\right\| .
\end{aligned}
$$

Hence from (114) and (117) we get

$$
\lim _{n \rightarrow \infty}\left\|v_{n}-G v_{n}\right\|=\lim _{n \rightarrow \infty}\left\|v_{n}-w_{n}\right\|=0 .
$$

Observe that

$$
\begin{aligned}
& \left\|v_{n}-T_{n} v_{n}\right\| \\
& \quad \leq\left\|v_{n}-x_{n}\right\|+\left\|x_{n}-T_{n} G v_{n}\right\|+\left\|T_{n} G v_{n}-T_{n} v_{n}\right\| \\
& \quad \leq\left\|v_{n}-x_{n}\right\|+\left\|x_{n}-T_{n} G v_{n}\right\|+\left\|G v_{n}-v_{n}\right\| .
\end{aligned}
$$

Hence, from (75), (104), and (119) we have

$$
\lim _{n \rightarrow \infty}\left\|v_{n}-T_{n} v_{n}\right\|=0
$$

It is clear that

$$
\begin{aligned}
\| P_{C} & \left(I-\lambda_{n} \nabla f\right) v_{n}-v_{n} \| \\
& =\left\|s_{n} v_{n}+\left(1-s_{n}\right) T_{n} v_{n}-v_{n}\right\| \\
& =\left(1-s_{n}\right)\left\|T_{n} v_{n}-v_{n}\right\| \\
& \leq\left\|T_{n} v_{n}-v_{n}\right\|,
\end{aligned}
$$


where $s_{n}=\left(2-\lambda_{n} L\right) / 4 \in(0,1 / 2)$ for each $\lambda_{n} \in(0,2 / L)$. Hence we have

$$
\begin{aligned}
\| P_{C}(I & \left.-\frac{2}{L} \nabla f\right) v_{n}-v_{n} \| \\
\leq & \left\|P_{C}\left(I-\frac{2}{L} \nabla f\right) v_{n}-P_{C}\left(I-\lambda_{n} \nabla f\right) v_{n}\right\| \\
& +\left\|P_{C}\left(I-\lambda_{n} \nabla f\right) v_{n}-v_{n}\right\| \\
\leq & \left\|\left(I-\frac{2}{L} \nabla f\right) v_{n}-\left(I-\lambda_{n} \nabla f\right) v_{n}\right\| \\
& +\left\|P_{C}\left(I-\lambda_{n} \nabla f\right) v_{n}-v_{n}\right\| \\
\leq & \left(\frac{2}{L}-\lambda_{n}\right)\left\|\nabla f\left(v_{n}\right)\right\|+\left\|T_{n} v_{n}-v_{n}\right\| .
\end{aligned}
$$

From the boundedness of $\left\{v_{n}\right\}, s_{n} \rightarrow 0\left(\Leftrightarrow \lambda_{n} \rightarrow 2 / L\right)$, and $\left\|T_{n} v_{n}-v_{n}\right\| \rightarrow 0$ (due to (121)), it follows that

$$
\lim _{n \rightarrow \infty}\left\|v_{n}-P_{C}\left(I-\frac{2}{L} \nabla f\right) v_{n}\right\|=0 .
$$

In addition, from (67) and (77), we have

$$
\begin{aligned}
\left\|z_{n+1}-z_{n}\right\| & \\
\leq\left\|z_{n+1}-x_{n+1}\right\|+\left\|x_{n+1}-x_{n}\right\|+\left\|x_{n}-z_{n}\right\| & \longrightarrow 0 \\
\text { as } n & \longrightarrow \infty .
\end{aligned}
$$

We note that

$$
\begin{aligned}
\| S^{n} z_{n}- & S^{n+1} z_{n} \| \\
\leq & \left\|S^{n} z_{n}-z_{n}\right\|+\left\|z_{n}-z_{n+1}\right\| \\
& +\left\|z_{n+1}-S^{n+1} z_{n+1}\right\|+\left\|S^{n+1} z_{n+1}-S^{n+1} z_{n}\right\| .
\end{aligned}
$$

From (81), (125), and Lemma 17, we obtain

$$
\lim _{n \rightarrow \infty}\left\|S^{n} z_{n}-S^{n+1} z_{n}\right\|=0 .
$$

In the meantime, we note that

$$
\begin{aligned}
\left\|z_{n}-S z_{n}\right\| \leq & \left\|z_{n}-S^{n} z_{n}\right\|+\left\|S^{n} z_{n}-S^{n+1} z_{n}\right\| \\
& +\left\|S^{n+1} z_{n}-S z_{n}\right\| .
\end{aligned}
$$

From (81), (127), and the uniform continuity of $S$, we have

$$
\lim _{n \rightarrow \infty}\left\|z_{n}-S z_{n}\right\|=0 .
$$

Step 4. We prove that $x_{n} \rightarrow x^{*}=P_{\Omega} x_{0}$ as $n \rightarrow \infty$.

Indeed, since $\left\{x_{n}\right\}$ is bounded, there exists a subsequence $\left\{x_{n_{i}}\right\}$ which converges weakly to some $w$. From (77), (83), (104), (92), and (102) we have that $z_{n_{i}} \rightarrow w, u_{n_{i}} \rightarrow w, v_{n_{i}} \rightarrow w$, $\Delta_{n_{i}}^{k} x_{n_{i}} \rightarrow w$, and $\Lambda_{n_{i}}^{m} u_{n_{i}} \rightarrow w$, where $k \in\{1,2, \ldots, M\}$ and $m \in\{1,2, \ldots, N\}$. Since $S$ is uniformly continuous, by (129) we get $\lim _{n \rightarrow \infty}\left\|z_{n}-S^{m} z_{n}\right\|=0$ for any $m \geq 1$. Hence from
Lemma 19, we obtain $w \in F i x(S)$. In the meantime, utilizing Lemma 11, we deduce from $v_{n_{i}} \rightarrow w, x_{n_{i}} \rightarrow w$, (119), and (124) that $w \in \operatorname{GSVI}(G)$ and $w \in \operatorname{Fix}\left(P_{C}(I-(2 / L) \nabla f)\right)=$ $\operatorname{VI}(C, \nabla f)=\Gamma$. Next we prove that $w \in \cap_{m=1}^{N} \operatorname{VI}\left(C, A_{m}\right)$. Let

$$
\widetilde{T}_{m} v= \begin{cases}A_{m} v+N_{C} v, & v \in C, \\ \emptyset, & v \notin C,\end{cases}
$$

where $m \in\{1,2, \ldots, N\}$. Let $(v, u) \in G\left(\widetilde{T}_{m}\right)$. Since $u-A_{m} v \in$ $N_{C} v$ and $\Lambda_{n}^{m} u_{n} \in C$, we have

$$
\left\langle v-\Lambda_{n}^{m} u_{n}, u-A_{m} v\right\rangle \geq 0 .
$$

On the other hand, from $\Lambda_{n}^{m} u_{n}=P_{C}\left(I-\lambda_{m, n} A_{m}\right) \Lambda_{n}^{m-1} u_{n}$ and $v \in C$, we have

$$
\left\langle v-\Lambda_{n}^{m} u_{n}, \Lambda_{n}^{m} u_{n}-\left(\Lambda_{n}^{m-1} u_{n}-\lambda_{m, n} A_{m} \Lambda_{n}^{m-1} u_{n}\right)\right\rangle \geq 0,
$$

and hence

$$
\left\langle v-\Lambda_{n}^{m} u_{n}, \frac{\Lambda_{n}^{m} u_{n}-\Lambda_{n}^{m-1} u_{n}}{\lambda_{m, n}}+A_{m} \Lambda_{n}^{m-1} u_{n}\right\rangle \geq 0 .
$$

Therefore we have

$$
\begin{aligned}
\left\langle v-\Lambda_{n_{i}}^{m} u_{n_{i}}, u\right\rangle & \\
\geq & \left\langle v-\Lambda_{n_{i}}^{m} u_{n_{i}}, A_{m} v\right\rangle \\
\geq & \left\langle v-\Lambda_{n_{i}}^{m} u_{n_{i}}, A_{m} v\right\rangle \\
& -\left\langle v-\Lambda_{n_{i}}^{m} u_{n_{i}}, \frac{\Lambda_{n_{i}}^{m} u_{n_{i}}-\Lambda_{n_{i}}^{m-1} u_{n_{i}}}{\lambda_{m, n_{i}}}+A_{m} \Lambda_{n_{i}}^{m-1} u_{n_{i}}\right\rangle \\
= & \left\langle v-\Lambda_{n_{i}}^{m} u_{n_{i}}, A_{m} v-A_{m} \Lambda_{n_{i}}^{m} u_{n_{i}}\right\rangle \\
& +\left\langle v-\Lambda_{n_{i}}^{m} u_{n_{i}}, A_{m} \Lambda_{n_{i}}^{m} u_{n_{i}}-A_{m} \Lambda_{n_{i}}^{m-1} u_{n_{i}}\right\rangle \\
& -\left\langle v-\Lambda_{n_{i}}^{m} u_{n_{i}}, \frac{\Lambda_{n_{i}}^{m} u_{n_{i}}-\Lambda_{n_{i}}^{m-1} u_{n_{i}}}{\lambda_{m, n_{i}}}\right\rangle \\
\geq & \left\langle v-\Lambda_{n_{i}}^{m} u_{n_{i}}, A_{m} \Lambda_{n_{i}}^{m} u_{n_{i}}-A_{m} \Lambda_{n_{i}}^{m-1} u_{n_{i}}\right\rangle \\
& -\left\langle v-\Lambda_{n_{i}}^{m} u_{n_{i}}, \frac{\Lambda_{n_{i}}^{m} u_{n_{i}}-\Lambda_{n_{i}}^{m-1} u_{n_{i}}}{\lambda_{m, n_{i}}}\right\rangle .
\end{aligned}
$$

From (102) and since $A_{m}$ is Lipschitz continuous, we obtain that $\lim _{n \rightarrow \infty}\left\|A_{m} \Lambda_{n}^{m} u_{n}-A_{m} \Lambda_{n}^{m-1} u_{n}\right\|=0$. From $\Lambda_{n_{i}}^{m} u_{n_{i}} \rightarrow$ $w,\left\{\lambda_{i, n}\right\} \subset\left[a_{i}, b_{i}\right] \subset\left(0,2 \eta_{i}\right), \forall i \in\{1,2, \ldots, N\}$, and (102), we have

$$
\langle v-w, u\rangle \geq 0 .
$$

Since $\widetilde{T}_{m}$ is maximal monotone, we have $w \in \widetilde{T}_{m}^{-1} 0$ and hence $w \in \operatorname{VI}\left(C, A_{m}\right), m=1,2, \ldots, N$, which implies $w \in \cap_{m=1}^{N} \operatorname{VI}\left(C, A_{m}\right)$. Next we prove that 
$w \in \cap_{k=1}^{M} \operatorname{GMEP}\left(\Theta_{k}, \varphi_{k}, B_{k}\right)$. Since $\Delta_{n}^{k} x_{n}=T_{r_{k, \mathrm{n}}}^{\left(\Theta_{k}, \varphi_{k}\right)}(I-$ $\left.r_{k, n} B_{k}\right) \Delta_{n}^{k-1} x_{n}, n \geq 1, k \in\{1,2, \ldots, M\}$, we have

$$
\begin{aligned}
& \Theta_{k}\left(\Delta_{n}^{k} x_{n}, y\right)+\varphi_{k}(y)-\varphi_{k}\left(\Delta_{n}^{k} x_{n}\right) \\
& +\left\langle B_{k} \Delta_{n}^{k-1} x_{n}, y-\Delta_{n}^{k} x_{n}\right\rangle \\
& \quad+\frac{1}{r_{k, n}}\left\langle y-\Delta_{n}^{k} x_{n}, \Delta_{n}^{k} x_{n}-\Delta_{n}^{k-1} x_{n}\right\rangle \geq 0 .
\end{aligned}
$$

By (A2), we have

$$
\begin{aligned}
& \varphi_{k}(y)-\varphi_{k}\left(\Delta_{n}^{k} x_{n}\right)+\left\langle B_{k} \Delta_{n}^{k-1} x_{n}, y-\Delta_{n}^{k} x_{n}\right\rangle \\
&+ \frac{1}{r_{k, n}}\left\langle y-\Delta_{n}^{k} x_{n}, \Delta_{n}^{k} x_{n}-\Delta_{n}^{k-1} x_{n}\right\rangle \\
& \geq \Theta_{k}\left(y, \Delta_{n}^{k} x_{n}\right) .
\end{aligned}
$$

Let $z_{t}=t y+(1-t) w$ for all $t \in(0,1]$ and $y \in C$. This implies that $z_{t} \in C$. Then, we have

$$
\begin{aligned}
\left\langle z_{t}\right. & \left.-\Delta_{n}^{k} x_{n}, B_{k} z_{t}\right\rangle \\
\geq & \varphi_{k}\left(\Delta_{n}^{k} x_{n}\right)-\varphi_{k}\left(z_{t}\right)+\left\langle z_{t}-\Delta_{n}^{k} x_{n}, B_{k} z_{t}\right\rangle \\
& -\left\langle z_{t}-\Delta_{n}^{k} x_{n}, B_{k} \Delta_{n}^{k-1} x_{n}\right\rangle \\
& -\left\langle z_{t}-\Delta_{n}^{k} x_{n}, \frac{\Delta_{n}^{k} x_{n}-\Delta_{n}^{k-1} x_{n}}{r_{k, n}}\right\rangle+\Theta_{k}\left(z_{t}, \Delta_{n}^{k} x_{n}\right) \\
= & \varphi_{k}\left(\Delta_{n}^{k} x_{n}\right)-\varphi_{k}\left(z_{t}\right)+\left\langle z_{t}-\Delta_{n}^{k} x_{n}, B_{k} z_{t}-B_{k} \Delta_{n}^{k} x_{n}\right\rangle \\
& +\left\langle z_{t}-\Delta_{n}^{k} x_{n}, B_{k} \Delta_{n}^{k} x_{n}-B_{k} \Delta_{n}^{k-1} x_{n}\right\rangle \\
& -\left\langle z_{t}-\Delta_{n}^{k} x_{n}, \frac{\Delta_{n}^{k} x_{n}-\Delta_{n}^{k-1} x_{n}}{r_{k, n}}\right\rangle+\Theta_{k}\left(z_{t}, \Delta_{n}^{k} x_{n}\right) .
\end{aligned}
$$

By (92), we have $\left\|B_{k} \Delta_{n}^{k} x_{n}-B_{k} \Delta_{n}^{k-1} x_{n}\right\| \rightarrow 0$ as $n \rightarrow \infty$. Furthermore, by the monotonicity of $B_{k}$, we obtain $\left\langle z_{t}-\right.$ $\left.\Delta_{n}^{k} x_{n}, B_{k} z_{t}-B_{k} \Delta_{n}^{k} x_{n}\right\rangle \geq 0$. Then, by (A4) we obtain

$$
\left\langle z_{t}-w, B_{k} z_{t}\right\rangle \geq \varphi_{k}(w)-\varphi_{k}\left(z_{t}\right)+\Theta_{k}\left(z_{t}, w\right) .
$$

Utilizing (A1), (A4), and (139), we obtain

$$
\begin{aligned}
0= & \Theta_{k}\left(z_{t}, z_{t}\right)+\varphi_{k}\left(z_{t}\right)-\varphi_{k}\left(z_{t}\right) \\
\leq & t \Theta_{k}\left(z_{t}, y\right)+(1-t) \Theta_{k}\left(z_{t}, w\right)+t \varphi_{k}(y) \\
& +(1-t) \varphi_{k}(w)-\varphi_{k}\left(z_{t}\right) \\
\leq & t\left[\Theta_{k}\left(z_{t}, y\right)+\varphi_{k}(y)-\varphi_{k}\left(z_{t}\right)\right] \\
& +(1-t)\left\langle z_{t}-w, B_{k} z_{t}\right\rangle \\
= & t\left[\Theta_{k}\left(z_{t}, y\right)+\varphi_{k}(y)-\varphi_{k}\left(z_{t}\right)\right] \\
& +(1-t) t\left\langle y-w, B_{k} z_{t}\right\rangle,
\end{aligned}
$$

and hence

$$
0 \leq \Theta_{k}\left(z_{t}, y\right)+\varphi_{k}(y)-\varphi_{k}\left(z_{t}\right)+(1-t)\left\langle y-w, B_{k} z_{t}\right\rangle .
$$

Letting $t \rightarrow 0$, we have, for each $y \in C$,

$$
0 \leq \Theta_{k}(w, y)+\varphi_{k}(y)-\varphi_{k}(w)+\left\langle y-w, B_{k} w\right\rangle .
$$

This implies that $w \in \operatorname{GMEP}\left(\Theta_{k}, \varphi_{k}, B_{k}\right)$ and hence $w \in \cap_{k=1}^{M} \operatorname{GMEP}\left(\Theta_{k}, \varphi_{k}, B_{k}\right)$. Consequently, $w \in \cap_{k=1}^{M} \operatorname{GMEP}\left(\Theta_{k}, \varphi_{k}, B_{k}\right) \cap \cap_{i=1}^{N} \operatorname{VI}\left(C, A_{i}\right) \cap \operatorname{GSVI}(G) \cap$ Fix $(S) \cap \Gamma=: \Omega$. This shows that $\omega_{w}\left(x_{n}\right) \subset \Omega$. From (64) and Lemma 22 we infer that $x_{n} \rightarrow x^{*}=P_{\Omega} x_{0}$ as $n \rightarrow \infty$.

Finally, assume additionally that $\left\|x_{n}-z_{n}\right\|=o\left(s_{n}\right)$ and $\lim _{n \rightarrow \infty} \sigma_{n}=0$. It is clear that

$$
\begin{array}{r}
\langle(V-I) x-(V-I) y, x-y\rangle \geq(\bar{\gamma}-1)\|x-y\|^{2}, \\
\forall x, y \in H .
\end{array}
$$

So, we know that $V-I$ is $(\bar{\gamma}-1)$-strongly monotone with constant $\bar{\gamma}-1>0$. In the meantime, it is easy to see that $V-I$ is $(\|V\|+1)$-Lipschitzian with constant $\|V\|+1>0$. Thus, there exists a unique solution $\widehat{x}$ in $\Omega$ to the VIP

$$
\langle(I-V) \hat{x}, p-\widehat{x}\rangle \leq 0, \quad \forall p \in \Omega .
$$

Equivalently, $\widehat{x}=P_{\Omega}(2 I-V) \widehat{x}$. Furthermore, from (58), (59), and (82) we get

$$
\begin{aligned}
\| z_{n}- & p \|^{2} \\
\leq & \left(\beta_{n}+s_{n} \bar{\gamma}\right)\left\|x_{n}-p\right\|^{2}+\left(1-\beta_{n}-s_{n} \bar{\gamma}\right) \\
& \times\left\|G v_{n}-p\right\|^{2} \\
& +2 s_{n}\left\langle(I-V) p+\sigma_{n}(\gamma Q-\mu F) p, z_{n}-p\right\rangle \\
\leq & \left(\beta_{n}+s_{n} \bar{\gamma}\right)\left\|x_{n}-p\right\|^{2}+\left(1-\beta_{n}-s_{n} \bar{\gamma}\right) \\
& \times\left\|v_{n}-p\right\|^{2} \\
& +2 s_{n}\left\langle(I-V) p+\sigma_{n}(\gamma Q-\mu F) p, z_{n}-p\right\rangle \\
\leq & \left(\beta_{n}+s_{n} \bar{\gamma}\right)\left\|x_{n}-p\right\|^{2}+\left(1-\beta_{n}-s_{n} \bar{\gamma}\right) \\
& \times\left\|x_{n}-p\right\|^{2} \\
& +2 s_{n}\left\langle(I-V) p+\sigma_{n}(\gamma Q-\mu F) p, z_{n}-p\right\rangle \\
= & \left\|x_{n}-p\right\|^{2} \\
& +2 s_{n}\left\langle(I-V) p+\sigma_{n}(\gamma Q-\mu F) p, z_{n}-p\right\rangle,
\end{aligned}
$$

which hence yields

$$
\begin{aligned}
& \left\langle(I-V) p+\sigma_{n}(\gamma Q-\mu F) p, p-z_{n}\right\rangle \\
& \quad \leq \frac{\left\|x_{n}-p\right\|^{2}-\left\|z_{n}-p\right\|^{2}}{2 s_{n}} \\
& \quad \leq \frac{\left\|x_{n}-z_{n}\right\|}{2 s_{n}}\left(\left\|x_{n}-p\right\|+\left\|z_{n}-p\right\|\right) .
\end{aligned}
$$


Since $\left\|x_{n}-z_{n}\right\|=o\left(s_{n}\right), \lim _{n \rightarrow \infty} \sigma_{n}=0, \lim _{n \rightarrow \infty}\left\|x_{n}-x^{*}\right\|=$ 0 , and $\left\{x_{n}\right\},\left\{z_{n}\right\}$ are bounded, we infer from (146) that

$$
\left\langle(I-V) p, p-x^{*}\right\rangle \leq 0, \quad \forall p \in \Omega,
$$

which, together with Minty's Lemma [4], implies that

$$
\left\langle(I-V) x^{*}, p-x^{*}\right\rangle \leq 0, \quad \forall p \in \Omega .
$$

This shows that $x^{*}$ is a solution in $\Omega$ to the VIP (144). Utilizing the uniqueness of solutions in $\Omega$ to the VIP (144), we get $x^{*}=$ $\widehat{x}$. This completes the proof.

Corollary 25. Let $C$ be a nonempty closed convex subset of a real Hilbert space $H$. Let $f: C \rightarrow \mathbf{R}$ be a convex functional with L-Lipschitz continuous gradient $\nabla f$. Let $\Theta$ be a bifunction from $C \times C$ to $\mathbf{R}$ satisfying (A1)-(A4) and let $\varphi: C \rightarrow \mathbf{R} \cup\{+\infty\}$ be a proper lower semicontinuous and convex function. Let $B, A_{i}: H \rightarrow H$, and $F_{j}: C \rightarrow H$ be $\zeta$ inverse-strongly monotone, $\eta_{i}$-inverse-strongly monotone, and $\zeta_{j}$-inverse-strongly monotone, respectively, for $i=1,2$ and $j=$ 1,2. Let $S: C \rightarrow C$ be a uniformly continuous asymptotically $k$-strict pseudocontractive mapping in the intermediate sense for some $0 \leq k<1$ with sequence $\left\{\gamma_{n}\right\} \subset[0, \infty)$ such that $\lim _{n \rightarrow \infty} \gamma_{n}=0$ and $\left\{c_{n}\right\} \subset[0, \infty)$ such that $\lim _{n \rightarrow \infty} c_{n}=0$. Let $V$ be a $\bar{\gamma}$-strongly positive bounded linear operator with $\bar{\gamma}>1$. Let $F: H \rightarrow H$ be a $\kappa$-Lipschitzian and $\eta$-strongly monotone operator with positive constants $\kappa, \eta>0$. Let $Q: H \rightarrow H$ be an l-Lipschitzian mapping with constant $l \geq 0$. Let $0<\mu<$ $2 \eta / \kappa^{2}$ and $0 \leq \gamma l \leq \tau$, where $\tau=1-\sqrt{1-\mu\left(2 \eta-\mu \kappa^{2}\right)}$. Assume that $\Omega:=\operatorname{GMEP}(\Theta, \varphi, B) \cap \operatorname{VI}\left(C, A_{1}\right) \cap \operatorname{VI}\left(C, A_{2}\right) \cap$ $\operatorname{GSVI}(G) \cap F i x(S) \cap \Gamma$ is nonempty and bounded and that either (B1) or (B2) holds. Let $0<\alpha \leq \alpha_{n} \leq 1, k \leq \delta_{n} \leq d<1$ for all $n \geq 1$, and let $\left\{\beta_{n}\right\},\left\{\sigma_{n}\right\}$ be sequences in $(0,1]$. Pick any $x_{0} \in H$ and set $C_{1}=C, x_{1}=P_{C_{1}} x_{0}$. Let $\left\{x_{n}\right\}$ be a sequence generated by the following algorithm:

$$
\begin{gathered}
\Theta\left(u_{n}, y\right)+\varphi(y)-\varphi\left(u_{n}\right)+\left\langle B x_{n}, y-u_{n}\right\rangle \\
+\frac{1}{r_{n}}\left\langle u_{n}-x_{n}, y-u_{n}\right\rangle \geq 0, \quad \forall y \in C, \\
v_{n}=P_{C}\left(I-\lambda_{2, n} A_{2}\right) P_{C}\left(I-\lambda_{1, n} A_{1}\right) u_{n}, \\
z_{n}=\beta_{n} x_{n}+\left(\left(1-\beta_{n}\right) I-s_{n} V\right) T_{n} G v_{n} \\
+s_{n}\left[T_{n} x_{n}-\sigma_{n}\left(\mu F\left(T_{n} x_{n}\right)-\gamma Q x_{n}\right)\right], \\
k_{n}=\delta_{n} z_{n}+\left(1-\delta_{n}\right) S^{n} z_{n}, \\
y_{n}=\left(1-\alpha_{n}\right) x_{n}+\alpha_{n} k_{n}, \\
C_{n+1}=\left\{z \in C_{n}:\left\|y_{n}-z\right\|^{2} \leq\left\|x_{n}-z\right\|^{2}+\theta_{n}\right\}, \\
x_{n+1}=P_{C_{n+1}} x_{0}, \quad \forall n \geq 1,
\end{gathered}
$$

where $P_{C}\left(I-\lambda_{n} \nabla f\right)=s_{n} I+\left(1-s_{n}\right) T_{n}$ (here $T_{n}$ is nonexpansive; $s_{n}=\left(2-\lambda_{n} L\right) / 4 \in(0,1 / 2)$ for each $\left.\lambda_{n} \in(0,2 / L)\right), \theta_{n}=$ $\left(s_{n}+\gamma_{n}\right)\left(1+\gamma_{n}\right) \Delta_{n}+c_{n}$, and $\Delta_{n}=\sup \left\{\left\|x_{n}-p\right\|^{2}+(\|(I-\right.$ $\left.V) p\|+\|(\gamma Q-\mu F) p \|)^{2} /(\bar{\gamma}-1): p \in \Omega\right\}<\infty$. Suppose that the following conditions are satisfied: (i) $s_{n} \in(0,1 / 2)$ for each $\lambda_{n} \in(0,2 / L), \lim _{n \rightarrow \infty} s_{n}=0$ $\left(\Leftrightarrow \lim _{n \rightarrow \infty} \lambda_{n}=2 / L\right)$;

(ii) $\left\{r_{n}\right\} \subset[e, f] \subset(0,2 \zeta),\left\{\lambda_{i, n}\right\} \subset\left[a_{i}, b_{i}\right] \subset\left(0,2 \eta_{i}\right)$, and $\nu_{j} \in\left(0,2 \zeta_{j}\right)$ for $i=1,2$ and $j=1,2$;

(iii) $0<\liminf _{n \rightarrow \infty} \beta_{n} \leq \limsup _{n \rightarrow \infty} \beta_{n}<1$.

Then one has the following:

(I) $\left\{x_{n}\right\}$ converges strongly as $\lambda_{n} \rightarrow(2 / L)\left(\Leftrightarrow s_{n} \rightarrow 0\right)$ to $x^{*}=P_{\Omega} x_{0}$;

(II) $\left\{x_{n}\right\}$ converges strongly as $\lambda_{n} \rightarrow(2 / L)\left(\Leftrightarrow s_{n} \rightarrow\right.$ 0) to $x^{*}=P_{\Omega} x_{0}$ provided $\left\|x_{n}-z_{n}\right\|=o\left(s_{n}\right)$ and $\lim _{n \rightarrow \infty} \sigma_{n}=0$, which is the unique solution in $\Omega$ to the VIP

$$
\left\langle(I-V) x^{*}, p-x^{*}\right\rangle \leq 0, \quad \forall p \in \Omega .
$$

Equivalently, $x^{*}=P_{\Omega}(2 I-V) x^{*}$.

Corollary 26. Let $C$ be a nonempty closed convex subset of a real Hilbert space $H$. Let $f: C \rightarrow \mathbf{R}$ be a convex functional with L-Lipschitz continuous gradient $\nabla f$. Let $\Theta$ be a bifunction from $C \times C$ to $\mathbf{R}$ satisfying (A1)-(A4) and let $\varphi: C \rightarrow \mathbf{R} \cup\{+\infty\}$ be a proper lower semicontinuous and convex function. Let $B, A: H \rightarrow H$, and $F_{j}: C \rightarrow H$ be $\zeta$-inverse-strongly monotone, $\xi$-inverse-strongly monotone, and $\zeta_{j}$-inverse-strongly monotone, respectively, for $j=1,2$. Let $S: C \rightarrow C$ be a uniformly continuous asymptotically $k$-strict pseudocontractive mapping in the intermediate sense for some $0 \leq k<1$ with sequence $\left\{\gamma_{n}\right\} \subset[0, \infty)$ such that $\lim _{n \rightarrow \infty} \gamma_{n}=$ 0 and $\left\{c_{n}\right\} \subset[0, \infty)$ such that $\lim _{n \rightarrow \infty} c_{n}=0$. Let $V$ be $a \bar{\gamma}-$ strongly positive bounded linear operator with $\bar{\gamma}>1$. Let $F$ : $H \rightarrow H$ be a $\kappa$-Lipschitzian and $\eta$-strongly monotone operator with positive constants $\kappa, \eta>0$. Let $Q: H \rightarrow H$ be an $l$ Lipschitzian mapping with constant $l \geq 0$. Let $0<\mu<2 \eta / \kappa^{2}$ and $0 \leq \gamma l \leq \tau$, where $\tau=1-\sqrt{1-\mu\left(2 \eta-\mu \kappa^{2}\right)}$. Assume that $\Omega:=\operatorname{GMEP}(\Theta, \varphi, B) \cap \operatorname{VI}(C, A) \cap \operatorname{GSVI}(G) \cap \operatorname{Fix}(S) \cap \Gamma$ is nonempty and bounded and that either (B1) or (B2) holds. Let $0<\alpha \leq \alpha_{n} \leq 1, k \leq \delta_{n} \leq d<1$ for all $n \geq 1$, and let $\left\{\beta_{n}\right\},\left\{\sigma_{n}\right\}$ be sequences in $(0,1]$. Pick any $x_{0} \in H$ and set $C_{1}=C, x_{1}=P_{C_{1}} x_{0}$. Let $\left\{x_{n}\right\}$ be a sequence generated by the following algorithm:

$$
\begin{gathered}
\Theta\left(u_{n}, y\right)+\varphi(y)-\varphi\left(u_{n}\right)+\left\langle B x_{n}, y-u_{n}\right\rangle \\
+\frac{1}{r_{n}}\left\langle u_{n}-x_{n}, y-u_{n}\right\rangle \geq 0, \quad \forall y \in C, \\
v_{n}=P_{C}\left(I-\rho_{n} A\right) u_{n}, \\
z_{n}=\beta_{n} x_{n}+\left(\left(1-\beta_{n}\right) I-s_{n} V\right) T_{n} G v_{n} \\
+s_{n}\left[T_{n} x_{n}-\sigma_{n}\left(\mu F\left(T_{n} x_{n}\right)-\gamma Q x_{n}\right)\right], \\
k_{n}=\delta_{n} z_{n}+\left(1-\delta_{n}\right) S^{n} z_{n}, \\
y_{n}=\left(1-\alpha_{n}\right) x_{n}+\alpha_{n} k_{n}, \\
C_{n+1}=\left\{z \in C_{n}:\left\|y_{n}-z\right\|^{2} \leq\left\|x_{n}-z\right\|^{2}+\theta_{n}\right\}, \\
x_{n+1}=P_{C_{n+1}} x_{0}, \quad \forall n \geq 1,
\end{gathered}
$$


where $P_{C}\left(I-\lambda_{n} \nabla f\right)=s_{n} I+\left(1-s_{n}\right) T_{n}$ (here $T_{n}$ is nonexpansive; $s_{n}=\left(2-\lambda_{n} L\right) / 4 \in(0,1 / 2)$ for each $\left.\lambda_{n} \in(0,2 / L)\right), \theta_{n}=$ $\left(s_{n}+\gamma_{n}\right)\left(1+\gamma_{n}\right) \Delta_{n}+c_{n}$, and $\Delta_{n}=\sup \left\{\left\|x_{n}-p\right\|^{2}+(\|(I-\right.$ $\left.V) p\|+\|(\gamma Q-\mu F) p \|)^{2} /(\bar{\gamma}-1): p \in \Omega\right\}<\infty$. Suppose that the following conditions are satisfied:

(i) $s_{n} \in(0,1 / 2)$ for each $\lambda_{n} \in(0,2 / L), \lim _{n \rightarrow \infty} s_{n}=0(\Leftrightarrow$ $\lim _{n \rightarrow \infty} \lambda_{n}=2 / L$ );

(ii) $\left\{r_{n}\right\} \subset[e, f] \subset(0,2 \zeta),\left\{\rho_{n}\right\} \subset[a, b] \subset(0,2 \xi)$, and $v_{j} \in\left(0,2 \zeta_{j}\right)$ for $j=1,2$;

(iii) $0<\liminf _{n \rightarrow \infty} \beta_{n} \leq \lim \sup _{n \rightarrow \infty} \beta_{n}<1$.

Then one has the following:

(I) $\left\{x_{n}\right\}$ converges strongly as $\lambda_{n} \rightarrow(2 / L)\left(\Leftrightarrow s_{n} \rightarrow 0\right)$ to $x^{*}=P_{\Omega} x_{0}$;

(II) $\left\{x_{n}\right\}$ converges strongly as $\lambda_{n} \rightarrow(2 / L)\left(\Leftrightarrow s_{n} \rightarrow\right.$ 0) to $x^{*}=P_{\Omega} x_{0}$ provided $\left\|x_{n}-z_{n}\right\|=o\left(s_{n}\right)$ and $\lim _{n \rightarrow \infty} \sigma_{n}=0$, which is the unique solution in $\Omega$ to the VIP

$$
\left\langle(I-V) x^{*}, p-x^{*}\right\rangle \leq 0, \quad \forall p \in \Omega .
$$

Equivalently, $x^{*}=P_{\Omega}(2 I-V) x^{*}$.

Corollary 27. Let $C$ be a nonempty closed convex subset of a real Hilbert space $H$. Let $f: C \rightarrow \mathbf{R}$ be a convex functional with L-Lipschitz continuous gradient $\nabla f$. Let $\Theta$ be a bifunction from $C \times C$ to $\mathbf{R}$ satisfying (A1)-(A4) and let $\varphi: C \rightarrow \mathbf{R} \cup\{+\infty\}$ be a proper lower semicontinuous and convex function. Let $B, A: H \rightarrow H$, and $F_{j}: C \rightarrow H$ be $\zeta$-inverse-strongly monotone, $\xi$-inverse-strongly monotone, and $\zeta_{j}$-inverse-strongly monotone, respectively, for $j=1,2$. Let $S: C \rightarrow C$ be a uniformly continuous asymptotically $k$-strict pseudocontractive mapping for some $0 \leq k<1$ with sequence $\left\{\gamma_{n}\right\} \subset[0, \infty)$ such that $\lim _{n \rightarrow \infty} \gamma_{n}=0$. Let $V$ be a $\bar{\gamma}$-strongly positive bounded linear operator with $\bar{\gamma}>1$. Let $F$ : $H \rightarrow H$ be $\alpha \kappa$-Lipschitzian and $\eta$-strongly monotone operator with positive constants $\kappa, \eta>0$. Let $\mathrm{Q}: H \rightarrow H$ be an $l$ Lipschitzian mapping with constant $l \geq 0$. Let $0<\mu<2 \eta / \kappa^{2}$ and $0 \leq \gamma l \leq \tau$, where $\tau=1-\sqrt{1-\mu\left(2 \eta-\mu \kappa^{2}\right)}$. Assume that $\Omega:=\operatorname{GMEP}(\Theta, \varphi, B) \cap V I(C, A) \cap G S V I(G) \cap F i x(S) \cap \Gamma$ is nonempty and bounded and that either (B1) or (B2) holds. Let $0<\alpha \leq \alpha_{n} \leq 1, k \leq \delta_{n} \leq d<1$ for all $n \geq 1$, and let $\left\{\beta_{n}\right\},\left\{\sigma_{n}\right\}$ be sequences in $(0,1]$. Pick any $x_{0} \in H$ and set $C_{1}=C, x_{1}=P_{C_{1}} x_{0}$. Let $\left\{x_{n}\right\}$ be a sequence generated by the following algorithm:

$$
\begin{gathered}
\Theta\left(u_{n}, y\right)+\varphi(y)-\varphi\left(u_{n}\right)+\left\langle B x_{n}, y-u_{n}\right\rangle \\
+\frac{1}{r_{n}}\left\langle u_{n}-x_{n}, y-u_{n}\right\rangle \geq 0, \quad \forall y \in C, \\
v_{n}=P_{C}\left(I-\rho_{n} A\right) u_{n},
\end{gathered}
$$

$$
\begin{gathered}
z_{n}=\beta_{n} x_{n}+\left(\left(1-\beta_{n}\right) I-s_{n} V\right) T_{n} G v_{n} \\
+s_{n}\left[T_{n} x_{n}-\sigma_{n}\left(\mu F\left(T_{n} x_{n}\right)-\gamma Q x_{n}\right)\right], \\
k_{n}=\delta_{n} z_{n}+\left(1-\delta_{n}\right) S^{n} z_{n}, \\
y_{n}=\left(1-\alpha_{n}\right) x_{n}+\alpha_{n} k_{n}, \\
C_{n+1}=\left\{z \in C_{n}:\left\|y_{n}-z\right\|^{2} \leq\left\|x_{n}-z\right\|^{2}+\theta_{n}\right\}, \\
x_{n+1}=P_{C_{n+1}} x_{0}, \quad \forall n \geq 1,
\end{gathered}
$$

where $P_{C}\left(I-\lambda_{n} \nabla f\right)=s_{n} I+\left(1-s_{n}\right) T_{n}$ (here $T_{n}$ is nonexpansive; $s_{n}=\left(2-\lambda_{n} L\right) / 4 \in(0,1 / 2)$ for each $\left.\lambda_{n} \in(0,2 / L)\right)$, $\theta_{n}=\left(s_{n}+\gamma_{n}\right)\left(1+\gamma_{n}\right) \Delta_{n}$, and $\Delta_{n}=\sup \left\{\left\|x_{n}-p\right\|^{2}+(\|(I-\right.$ $\left.V) p\|+\|(\gamma Q-\mu F) p \|)^{2} /(\bar{\gamma}-1): p \in \Omega\right\}<\infty$. Suppose that the following conditions are satisfied:

(i) $s_{n} \in(0,1 / 2)$ for each $\lambda_{n} \in(0,2 / L), \lim _{n \rightarrow \infty} s_{n}=0$ $\left(\Leftrightarrow \lim _{n \rightarrow \infty} \lambda_{n}=2 / L\right)$;

(ii) $\left\{r_{n}\right\} \subset[e, f] \subset(0,2 \zeta),\left\{\rho_{n}\right\} \subset[a, b] \subset(0,2 \xi)$, and $v_{j} \in\left(0,2 \zeta_{j}\right)$ for $j=1,2$;

(iii) $0<\liminf _{n \rightarrow \infty} \beta_{n} \leq \lim \sup _{n \rightarrow \infty} \beta_{n}<1$.

Then one has the following:

(I) $\left\{x_{n}\right\}$ converges strongly as $\lambda_{n} \rightarrow(2 / L)\left(\Leftrightarrow s_{n} \rightarrow 0\right)$ to $x^{*}=P_{\Omega} x_{0}$;

(II) $\left\{x_{n}\right\}$ converges strongly as $\lambda_{n} \rightarrow(2 / L)\left(\Leftrightarrow s_{n} \rightarrow\right.$ 0) to $x^{*}=P_{\Omega} x_{0}$ provided $\left\|x_{n}-z_{n}\right\|=o\left(s_{n}\right)$ and $\lim _{n \rightarrow \infty} \sigma_{n}=0$, which is the unique solution in $\Omega$ to the VIP

$$
\left\langle(I-V) x^{*}, p-x^{*}\right\rangle \leq 0, \quad \forall p \in \Omega .
$$

Equivalently, $x^{*}=P_{\Omega}(2 I-V) x^{*}$.

\section{Fixed Point Problems with Constraints}

In this section, we will introduce and analyze another implicit iterative algorithm for solving the fixed point problem of infinitely many nonexpansive mappings with constraints of several problems: finitely many GMEPs, finitely many VIPs, the GSVI (8), and the fixed point problem of an asymptotically strict pseudocontractive mapping in the intermediate sense in a real Hilbert space. We prove strong convergence theorem for the iterative algorithm under mild assumptions. This iterative algorithm is based on shrinking projection method, Korpelevich's extragradient method, hybrid steepest-descent method in [7], viscosity approximation method, $W$-mapping approach to fixed points of infinitely many nonexpansive mappings, and strongly positive bounded linear operator technique.

Theorem 28. Let $C$ be a nonempty closed convex subset of a real Hilbert space $H$. Let $M, N$ be two integers. Let $\Theta_{k}$ be a bifunction from $C \times C$ to $\mathbf{R}$ satisfying (A1)-(A4) and let $\varphi_{k}: C \rightarrow \mathbf{R} \cup\{+\infty\}$ be a proper lower semicontinuous 
and convex function, where $k \in\{1,2, \ldots, M\}$. Let $B_{k}, A_{i}$ : $H \rightarrow H$, and $F_{j}: C \rightarrow H$ be $\mu_{k}$-inverse-strongly monotone, $\eta_{i}$-inverse-strongly monotone, and $\zeta_{j}$-inverse-strongly monotone, respectively, where $k \in\{1,2, \ldots, M\}, i \in\{1,2, \ldots, N\}$, and $j \in\{1,2\}$. Let $\left\{T_{n}\right\}_{n=1}^{\infty}$ be a sequence of nonexpansive mappings on $H$ and let $\left\{\lambda_{n}\right\}$ be a sequence in $(0, b]$ for some $b \in(0,1)$. Let $S: C \rightarrow C$ be a uniformly continuous asymptotically $k$-strict pseudocontractive mapping in the intermediate sense for some $0 \leq k<1$ with sequence $\left\{\gamma_{n}\right\} \subset[0, \infty)$ such that $\lim _{n \rightarrow \infty} \gamma_{n}=0$ and $\left\{c_{n}\right\} \subset[0, \infty)$ such that $\lim _{n \rightarrow \infty} c_{n}=0$. Let $V$ be a $\bar{\gamma}$-strongly positive bounded linear operator with $\bar{\gamma}>1$. Let $F: H \rightarrow H$ be a $\kappa$ Lipschitzian and $\eta$-strongly monotone operator with positive constants $\kappa, \eta>0$. Let $Q: H \rightarrow H$ be an l-Lipschitzian mapping with constant $l \geq 0$. Let $0<\mu<2 \eta / \kappa^{2}$ and $0 \leq \gamma l \leq \tau$, where $\tau=1-\sqrt{1-\mu\left(2 \eta-\mu \kappa^{2}\right)}$. Assume that $\Omega:=\cap_{n=1}^{\infty} \operatorname{Fix}\left(T_{n}\right) \cap \cap_{k=1}^{M} \operatorname{GMEP}\left(\Theta_{k}, \varphi_{k}, B_{k}\right) \cap \cap_{i=1}^{N} V I\left(C, A_{i}\right) \cap$ $\operatorname{GSVI}(G) \cap \operatorname{Fix}(S)$ is nonempty and bounded and that either (B1) or (B2) holds. Let $0<\alpha \leq \alpha_{n} \leq 1, k \leq \delta_{n} \leq d<1$ for all $n \geq 1$, and let $\left\{\beta_{n}\right\},\left\{\epsilon_{n}\right\}$, and $\left\{\sigma_{n}\right\}$ be sequences in $(0,1]$. Pick any $x_{0} \in H$ and set $C_{1}=C, x_{1}=P_{C_{1}} x_{0}$. Let $\left\{x_{n}\right\}$ be a sequence generated by the following algorithm:

$$
\begin{aligned}
& u_{n}=T_{r_{M, n}}^{\left(\Theta_{M}, \varphi_{M}\right)}\left(I-r_{M, n} B_{M}\right) T_{r_{M-1, n}}^{\left(\Theta_{M-1}, \varphi_{M-1}\right)} \\
& \times\left(I-r_{M-1, n} B_{M-1}\right) \cdots T_{r_{1, n}}^{\left(\Theta_{1}, \varphi_{1}\right)}\left(I-r_{1, n} B_{1}\right) x_{n}, \\
& v_{n}=P_{C}\left(I-\lambda_{N, n} A_{N}\right) P_{C}\left(I-\lambda_{N-1, n} A_{N-1}\right) \cdots P_{C} \\
& \times\left(I-\lambda_{2, n} B_{2}\right) P_{C}\left(I-\lambda_{1, n} B_{1}\right) u_{n}, \\
& z_{n}=\beta_{n} x_{n}+\left(\left(1-\beta_{n}\right) I-\epsilon_{n} V\right) W_{n} G v_{n} \\
& +\epsilon_{n}\left[W_{n} x_{n}-\sigma_{n}\left(\mu F\left(W_{n} x_{n}\right)-\gamma Q x_{n}\right)\right], \\
& k_{n}=\delta_{n} z_{n}+\left(1-\delta_{n}\right) S^{n} z_{n} \\
& y_{n}=\left(1-\alpha_{n}\right) x_{n}+\alpha_{n} k_{n}, \\
& C_{n+1}=\left\{z \in C_{n}:\left\|y_{n}-z\right\|^{2} \leq\left\|x_{n}-z\right\|^{2}+\theta_{n}\right\} \text {, } \\
& x_{n+1}=P_{C_{n+1}} x_{0}, \quad \forall n \geq 1 \text {, }
\end{aligned}
$$

where $W_{n}$ is the $W$-mapping defined by (34), $\theta_{n}=\left(\epsilon_{n}+\gamma_{n}\right)(1+$ $\left.\gamma_{n}\right) \Delta_{n}+c_{n}$, and $\Delta_{n}=\sup \left\{\left\|x_{n}-p\right\|^{2}+(\|(I-V) p\|+\|(\gamma Q-\right.$ $\left.\mu F) p \|)^{2} /(\bar{\gamma}-1): p \in \Omega\right\}<\infty$. Suppose that the following conditions are satisfied:

(i) $\left\{r_{k, n}\right\} \subset\left[e_{k}, f_{k}\right] \subset\left(0,2 \mu_{k}\right),\left\{\lambda_{i, n}\right\} \subset\left[a_{i}, b_{i}\right] \subset\left(0,2 \eta_{i}\right)$, and $v_{j} \in\left(0,2 \zeta_{j}\right)$, where $k \in\{1,2, \ldots, M\}, i \in$ $\{1,2, \ldots, N\}$, and $j \in\{1,2\}$;

(ii) $\lim _{n \rightarrow \infty} \epsilon_{n}=0$ and $0<\liminf _{n \rightarrow \infty} \beta_{n} \leq$ $\lim \sup _{n \rightarrow \infty} \beta_{n}<1$.

Then one has the following:

(I) $\left\{x_{n}\right\}$ converges strongly to $x^{*}=P_{\Omega} x_{0}$;
(II) $\left\{x_{n}\right\}$ converges strongly to $x^{*}=P_{\Omega} x_{0}$ provided $\| x_{n}-$ $z_{n} \|=o\left(\epsilon_{n}\right)$ and $\lim _{n \rightarrow \infty} \sigma_{n}=0$, which is the unique solution in $\Omega$ to the VIP

$$
\left\langle(I-V) x^{*}, p-x^{*}\right\rangle \leq 0, \quad \forall p \in \Omega \text {. }
$$

Equivalently, $x^{*}=P_{\Omega}(2 I-V) x^{*}$.

Proof. First of all, let us show that the sequence $\left\{x_{n}\right\}$ is well defined. As $\lim _{n \rightarrow \infty} \epsilon_{n}=0$ and $0<\liminf _{n \rightarrow \infty} \beta_{n} \leq$ $\limsup \sup _{n \rightarrow \infty} \beta_{n}<1$, we may assume, without loss of generality, that $\left\{\beta_{n}\right\} \subset[a, \widehat{a}] \subset(0,1)$ and $\beta_{n}+\epsilon_{n}\|V\| \leq 1$ for all $n \geq 1$. Utilizing the arguments similar to those in the proof of Theorem 24, we get

$$
\left\|\left(1-\beta_{n}\right) I-\epsilon_{n} V\right\| \leq 1-\beta_{n}-\epsilon_{n} \bar{\gamma}
$$

Put

$$
\begin{aligned}
\Delta_{n}^{k}= & T_{r_{k, n}}^{\left(\Theta_{k}, \varphi_{k}\right)}\left(I-r_{k, n} B_{k}\right) T_{r_{k-1, n}}^{\left(\Theta_{k-1}, \varphi_{k-1}\right)} \\
& \times\left(I-r_{k-1, n} B_{k-1}\right) \cdots T_{r_{1, n}}^{\left(\Theta_{1}, \varphi_{1}\right)}\left(I-r_{1, n} B_{1}\right) x_{n}
\end{aligned}
$$

for all $k \in\{1,2, \ldots, M\}$ and $n \geq 1$ and

$$
\Lambda_{n}^{i}=P_{C}\left(I-\lambda_{i, n} B_{i}\right) P_{C}\left(I-\lambda_{i-1, n} B_{i-1}\right) \cdots P_{C}\left(I-\lambda_{1, n} B_{1}\right)
$$

for all $i \in\{1,2, \ldots, N\}, \Delta_{n}^{0}=I$, and $\Lambda_{n}^{0}=I$, where $I$ is the identity mapping on $H$. Then we have that $u_{n}=\Delta_{n}^{M} x_{n}$ and $v_{n}=\Lambda_{n}^{N} u_{n}$.

We divide the rest of the proof into several steps.

Step 1. We show that $\left\{x_{n}\right\}$ is well defined. It is obvious that $C_{n}$ is closed and convex. As the defining inequality in $C_{n}$ is equivalent to the inequality

$$
\left\langle 2\left(x_{n}-y_{n}\right), z\right\rangle \leq\left\|x_{n}\right\|^{2}-\left\|y_{n}\right\|^{2}+\theta_{n}
$$

by Lemma 16 we know that $C_{n}$ is convex for every $n \geq 1$.

First of all, let us show that $\Omega \subset C_{n}$ for all $n \geq 1$. Suppose that $\Omega \subset C_{n}$ for some $n \geq 1$. Take $p \in \Omega$ arbitrarily. Utilizing the arguments similar to those in the proof of Theorem 24 we obtain that

$$
\begin{gathered}
\left\|u_{n}-p\right\| \leq\left\|\Delta_{n}^{k} x_{n}-\Delta_{n}^{k} p\right\| \leq\left\|x_{n}-p\right\|, \\
\left\|v_{n}-p\right\| \leq\left\|\Lambda_{n}^{i} u_{n}-\Lambda_{n}^{i} p\right\| \leq\left\|u_{n}-p\right\|, \\
\left\|v_{n}-p\right\| \leq\left\|x_{n}-p\right\|,
\end{gathered}
$$




$$
\begin{aligned}
\| G v_{n} & -p \|^{2} \\
\leq & \left\|P_{C}\left(I-v_{2} F_{2}\right) v_{n}-P_{C}\left(I-v_{2} F_{2}\right) p\right\|^{2} \\
& +v_{1}\left(v_{1}-2 \zeta_{1}\right) \\
& \times\left\|F_{1} P_{C}\left(I-v_{2} F_{2}\right) v_{n}-F_{1} P_{C}\left(I-v_{2} F_{2}\right) p\right\|^{2} \quad(164) \\
\leq & \left\|v_{n}-p\right\|^{2}+v_{2}\left(v_{2}-2 \zeta_{2}\right)\left\|F_{2} v_{n}-F_{2} p\right\|^{2} \\
\leq & \left\|v_{n}-p\right\|^{2}, \\
\| k_{n}- & p \|^{2} \\
\leq & \left(1+\gamma_{n}\right)\left\|z_{n}-p\right\|^{2} \\
& +\left(1-\delta_{n}\right)\left(k-\delta_{n}\right)\left\|z_{n}-S^{n} z_{n}\right\|^{2}+c_{n} \\
\leq & \left(1+\gamma_{n}\right)\left\|z_{n}-p\right\|^{2}+c_{n} \\
\leq & \left(1+\gamma_{n}\right) \\
& \times\left(\left\|x_{n}-p\right\|^{2}+\epsilon_{n} \frac{\left(\|(I-V) p\|+\|(\gamma Q-\mu F) p\|^{2}\right.}{\bar{\gamma}-1}\right) \\
& +c_{n} .
\end{aligned}
$$

$$
\begin{aligned}
& \leq\left\|x_{n}-p\right\|^{2}+\left(\epsilon_{n}+\gamma_{n}\right)\left(1+\gamma_{n}\right) \Delta_{n}+c_{n} \\
& =\left\|x_{n}-p\right\|^{2}+\theta_{n},
\end{aligned}
$$

where $\theta_{n}=\left(\epsilon_{n}+\gamma_{n}\right)\left(1+\gamma_{n}\right) \Delta_{n}+c_{n}$ and $\Delta_{n}=\sup \left\{\left\|x_{n}-p\right\|^{2}+\right.$ $\left.(\|(I-V) p\|+\|(\gamma Q-\mu F) p\|)^{2} /(\bar{\gamma}-1): p \in \Omega\right\}<\infty$. Hence $p \in C_{n+1}$. This implies that $\Omega \subset C_{n}$ for all $n \geq 1$. Therefore, $\left\{x_{n}\right\}$ is well defined.

Step 2. We prove that $\left\|x_{n}-k_{n}\right\| \rightarrow 0,\left\|x_{n}-z_{n}\right\| \rightarrow 0$, and $\left\|S^{n} z_{n}-z_{n}\right\| \rightarrow 0$ as $n \rightarrow \infty$.

Indeed, let $x^{*}=P_{\Omega} x_{0}$. From $x_{n}=P_{C_{n}} x_{0}$ and $x^{*} \in \Omega \subset$ $C_{n}$, we obtain

$$
\left\|x_{n}-x_{0}\right\| \leq\left\|x^{*}-x_{0}\right\| .
$$

This implies that $\left\{x_{n}\right\}$ is bounded and hence $\left\{u_{n}\right\},\left\{v_{n}\right\},\left\{z_{n}\right\},\left\{k_{n}\right\}$, and $\left\{y_{n}\right\}$ are also bounded. Utilizing the arguments similar to those of (67), (75), (77), and (81) in the proof of Theorem 24 we obtain that

$$
\begin{gathered}
\lim _{n \rightarrow \infty}\left\|x_{n+1}-x_{n}\right\|=0, \\
\lim _{n \rightarrow \infty}\left\|x_{n}-W_{n} G v_{n}\right\|=0, \\
\lim _{n \rightarrow \infty}\left\|x_{n}-z_{n}\right\|=0, \\
\lim _{n \rightarrow \infty}\left\|S^{n} z_{n}-z_{n}\right\|=0 .
\end{gathered}
$$

Step 3. We prove that $\left\|x_{n}-u_{n}\right\| \rightarrow 0,\left\|x_{n}-v_{n}\right\| \rightarrow 0, \| v_{n}-$ $G v_{n}\|\rightarrow 0,\| v_{n}-W v_{n} \| \rightarrow 0$, and $\left\|z_{n}-S z_{n}\right\| \rightarrow 0$ as $n \rightarrow \infty$. Indeed, from (162), (164), $\bar{\gamma}>1$, and $\gamma l \leq \tau$, it follows that

$$
\begin{aligned}
& \left\|z_{n}-p\right\|^{2} \\
& =\| \beta_{n}\left(x_{n}-p\right)+\left(\left(1-\beta_{n}\right) I-s_{n} V\right)\left(W_{n} G v_{n}-p\right) \\
& +s_{n}\left[\sigma_{n} \gamma\left(Q x_{n}-Q p\right)+\left(I-\sigma_{n} \mu F\right) W_{n} x_{n}\right. \\
& \left.\quad-\left(I-\sigma_{n} \mu F\right) W_{n} p\right] \\
& \quad+s_{n}\left[(I-V) p+\sigma_{n}(\gamma Q-\mu F) p\right] \|^{2} \\
& \leq\left[\beta_{n}\left\|x_{n}-p\right\|+\left\|\left(1-\beta_{n}\right) I-\epsilon_{n} V\right\|\left\|W_{n} G v_{n}-p\right\|\right. \\
& +\epsilon_{n}\left(\sigma_{n} \gamma\left\|Q x_{n}-Q p\right\|\right. \\
& \left.\left.\quad+\left\|\left(I-\sigma_{n} \mu F\right) W_{n} x_{n}-\left(I-\sigma_{n} \mu F\right) W_{n} p\right\|\right)\right]^{2} \\
& +2 \epsilon_{n}\left\langle(I-V) p+\sigma_{n}(\gamma Q-\mu F) p, z_{n}-p\right\rangle
\end{aligned}
$$




$$
\begin{aligned}
\leq & {\left[\beta_{n}\left\|x_{n}-p\right\|+\left(1-\beta_{n}-\epsilon_{n} \bar{\gamma}\right)\left\|G v_{n}-p\right\|\right.} \\
& \left.+\epsilon_{n}\left(\sigma_{n} \gamma l\left\|x_{n}-p\right\|+\left(1-\sigma_{n} \tau\right)\left\|x_{n}-p\right\|\right)\right]^{2} \\
& +2 \epsilon_{n}\left\langle(I-V) p+\sigma_{n}(\gamma Q-\mu F) p, z_{n}-p\right\rangle \\
= & {\left[\beta_{n}\left\|x_{n}-p\right\|+\left(1-\beta_{n}-\epsilon_{n} \bar{\gamma}\right)\left\|G v_{n}-p\right\|\right.} \\
& \left.+\epsilon_{n}\left(1-\sigma_{n}(\tau-\gamma l)\right)\left\|x_{n}-p\right\|\right]^{2} \\
& +2 \epsilon_{n}\left\langle(I-V) p+\sigma_{n}(\gamma Q-\mu F) p, z_{n}-p\right\rangle \\
\leq & {\left[\left(\beta_{n}+\epsilon_{n} \bar{\gamma}\right)\left\|x_{n}-p\right\|+\left(1-\beta_{n}-\epsilon_{n} \bar{\gamma}\right)\left\|G v_{n}-p\right\|\right]^{2} } \\
& +2 \epsilon_{n}\left\langle(I-V) p+\sigma_{n}(\gamma Q-\mu F) p, z_{n}-p\right\rangle \\
\leq & \left(\beta_{n}+\epsilon_{n} \bar{\gamma}\right)\left\|x_{n}-p\right\|^{2}+\left(1-\beta_{n}-\epsilon_{n} \bar{\gamma}\right)\left\|G v_{n}-p\right\|^{2} \\
& +2 \epsilon_{n}\left\langle(I-V) p+\sigma_{n}(\gamma Q-\mu F) p, z_{n}-p\right\rangle \\
\leq & \left(\beta_{n}+\epsilon_{n} \bar{\gamma}\right)\left\|x_{n}-p\right\|^{2}+\left(1-\beta_{n}-\epsilon_{n} \bar{\gamma}\right)\left\|v_{n}-p\right\|^{2} \\
& +2 \epsilon_{n}\left\langle(I-V) p+\sigma_{n}(\gamma Q-\mu F) p, z_{n}-p\right\rangle \\
\leq & \left(\beta_{n}+\epsilon_{n} \bar{\gamma}\right)\left\|x_{n}-p\right\|^{2}+\left(1-\beta_{n}-\epsilon_{n} \bar{\gamma}\right)\left\|u_{n}-p\right\|^{2} \\
& +2 \epsilon_{n}\left\langle(I-V) p+\sigma_{n}(\gamma Q-\mu F) p, z_{n}-p\right\rangle .
\end{aligned}
$$

Utilizing the arguments similar to those of (83), (92), (102), (104), (119), (121), and (129) in the proof of Theorem 24 we obtain that

$$
\begin{aligned}
\lim _{n \rightarrow \infty}\left\|x_{n}-u_{n}\right\| & =0, \\
\lim _{n \rightarrow \infty}\left\|\Delta_{n}^{k-1} x_{n}-\Delta_{n}^{k} x_{n}\right\|=0, \quad k & =1,2, \ldots, M, \\
\lim _{n \rightarrow \infty}\left\|\Lambda_{n}^{i-1} u_{n}-\Lambda_{n}^{i} u_{n}\right\|=0, \quad i & =1,2, \ldots, N, \\
\lim _{n \rightarrow \infty}\left\|x_{n}-v_{n}\right\| & =0, \\
\lim _{n \rightarrow \infty}\left\|v_{n}-G v_{n}\right\| & =0, \\
\lim _{n \rightarrow \infty}\left\|v_{n}-W v_{n} v_{n}\right\| & =0, \\
\lim _{n \rightarrow \infty}\left\|z_{n}-S z_{n}\right\| & =0 .
\end{aligned}
$$

In addition, note that

$$
\left\|v_{n}-W v_{n}\right\| \leq\left\|v_{n}-W_{n} v_{n}\right\|+\left\|W_{n} v_{n}-W v_{n}\right\| .
$$

So, from $\left\|v_{n}-W_{n} v_{n}\right\| \rightarrow 0$ and [20, Remark 3.2] it follows that

$$
\lim _{n \rightarrow \infty}\left\|v_{n}-W v_{n}\right\|=0
$$

Step 4 . We prove that $x_{n} \rightarrow x^{*}=P_{\Omega} x_{0}$ as $n \rightarrow \infty$.

Indeed, since $\left\{x_{n}\right\}$ is bounded, there exists a subsequence $\left\{x_{n_{i}}\right\}$ which converges weakly to some $w$. From (169), (172),
(175), (173), and (174) we have that $z_{n_{i}} \rightarrow w, u_{n_{i}} \rightarrow w, v_{n_{i}} \rightarrow$ $w, \Delta_{n_{i}}^{k} x_{n_{i}} \rightarrow w$, and $\Lambda_{n_{i}}^{m} u_{n_{i}} \rightarrow w$, where $k \in\{1,2, \ldots, M\}$ and $m \in\{1,2, \ldots, N\}$. Since $S$ is uniformly continuous, by (178) we get $\lim _{n \rightarrow \infty}\left\|z_{n}-S^{m} z_{n}\right\|=0$ for any $m \geq 1$. Hence, from Lemma 19, we obtain $w \in \operatorname{Fix}(S)$. In the meantime, utilizing Lemma 11, we deduce from (176) and (180) that $w \in$ $\operatorname{GSVI}(G)$ and $w \in \operatorname{Fix}(W)=\cap_{n=1}^{\infty} F i x\left(T_{n}\right)$ (due to Lemma 13). Hence we get $w \in \operatorname{GSVI}(G) \cap \cap_{n=1}^{\infty}$ Fix $\left(T_{n}\right)$. Repeating the same arguments as in the proof of Theorem 24 we conclude that $w \in \cap_{m=1}^{N} \operatorname{VI}\left(C, A_{m}\right)$ and $w \in \cap_{k=1}^{M} \operatorname{GMEP}\left(\Theta_{k}, \varphi_{k}, B_{k}\right)$. Consequently, $w \in \cap_{n=1}^{\infty} \operatorname{Fix}\left(T_{n}\right) \cap \cap_{k=1}^{M} \operatorname{GMEP}\left(\Theta_{k}, \varphi_{k}, B_{k}\right) \cap$ $\cap_{i=1}^{N} \operatorname{VI}\left(C, A_{i}\right) \cap \operatorname{GSVI}(G) \cap \operatorname{Fix}(S)=: \Omega$. This shows that $\omega_{w}\left(x_{n}\right) \subset \Omega$. From (167) and Lemma 22 we infer that $x_{n} \rightarrow$ $x^{*}=P_{\Omega} x_{0}$ as $n \rightarrow \infty$.

Finally, assume additionally that $\left\|x_{n}-z_{n}\right\|=o\left(\epsilon_{n}\right)$ and $\lim _{n \rightarrow \infty} \sigma_{n}=0$. It is clear that

$$
\begin{array}{r}
\langle(V-I) x-(V-I) y, x-y\rangle \geq(\bar{\gamma}-1)\|x-y\|^{2}, \\
\forall x, y \in H .
\end{array}
$$

So, we know that $V-I$ is $(\bar{\gamma}-1)$-strongly monotone with constant $\bar{\gamma}-1>0$. In the meantime, it is easy to see that $V-I$ is $(\|V\|+1)$-Lipschitzian with constant $\|V\|+1>0$. Thus, there exists a unique solution $\widehat{x}$ in $\Omega$ to the VIP

$$
\langle(I-V) \hat{x}, p-\hat{x}\rangle \leq 0, \quad \forall p \in \Omega .
$$

Equivalently, $\widehat{x}=P_{\Omega}(2 I-V) \widehat{x}$. Furthermore, from (163), (164), and (171) we get

$$
\begin{aligned}
&\left\|z_{n}-p\right\|^{2} \\
& \leq\left(\beta_{n}+\epsilon_{n} \bar{\gamma}\right)\left\|x_{n}-p\right\|^{2}+\left(1-\beta_{n}-\epsilon_{n} \bar{\gamma}\right)\left\|G v_{n}-p\right\|^{2} \\
&+2 \epsilon_{n}\left\langle(I-V) p+\sigma_{n}(\gamma Q-\mu F) p, z_{n}-p\right\rangle \\
& \leq\left(\beta_{n}+\epsilon_{n} \bar{\gamma}\right)\left\|x_{n}-p\right\|^{2} \\
&+\left(1-\beta_{n}-\epsilon_{n} \bar{\gamma}\right)\left\|v_{n}-p\right\|^{2} \\
&+2 \epsilon_{n}\left\langle(I-V) p+\sigma_{n}(\gamma Q-\mu F) p, z_{n}-p\right\rangle \\
& \leq\left(\beta_{n}+\epsilon_{n} \bar{\gamma}\right)\left\|x_{n}-p\right\|^{2}+\left(1-\beta_{n}-\epsilon_{n} \bar{\gamma}\right)\left\|x_{n}-p\right\|^{2} \\
&+2 \epsilon_{n}\left\langle(I-V) p+\sigma_{n}(\gamma Q-\mu F) p, z_{n}-p\right\rangle \\
&=\left\|x_{n}-p\right\|^{2} \\
&+2 \epsilon_{n}\left\langle(I-V) p+\sigma_{n}(\gamma Q-\mu F) p, z_{n}-p\right\rangle,
\end{aligned}
$$

which hence yields

$$
\begin{aligned}
& \left\langle(I-V) p+\sigma_{n}(\gamma Q-\mu F) p, p-z_{n}\right\rangle \\
& \quad \leq \frac{\left\|x_{n}-p\right\|^{2}-\left\|z_{n}-p\right\|^{2}}{2 \epsilon_{n}} \\
& \quad \leq \frac{\left\|x_{n}-z_{n}\right\|}{2 \epsilon_{n}}\left(\left\|x_{n}-p\right\|+\left\|z_{n}-p\right\|\right) .
\end{aligned}
$$


Since $\left\|x_{n}-z_{n}\right\|=o\left(s_{n}\right), \lim _{n \rightarrow \infty} \sigma_{n}=0, \lim _{n \rightarrow \infty}\left\|x_{n}-x^{*}\right\|=0$, and $\left\{x_{n}\right\},\left\{z_{n}\right\}$ are bounded, we infer from (94) that

$$
\left\langle(I-V) p, p-x^{*}\right\rangle \leq 0, \quad \forall p \in \Omega,
$$

which, together with Minty's Lemma, implies that

$$
\left\langle(I-V) x^{*}, p-x^{*}\right\rangle \leq 0, \quad \forall p \in \Omega .
$$

This shows that $x^{*}$ is a solution in $\Omega$ to the VIP (182). Utilizing the uniqueness of solutions in $\Omega$ to the VIP (182), we get $x^{*}=$ $\widehat{x}$. This completes the proof.

Corollary 29. Let $C$ be a nonempty closed convex subset of a real Hilbert space $H$. Let $\Theta$ be a bifunction from $C \times C$ to $\mathbf{R}$ satisfying (A1)-(A4) and let $\varphi: C \rightarrow \mathbf{R} \cup\{+\infty\}$ be a proper lower semicontinuous and convex function. Let $B, A_{i}: H \rightarrow$ $H$, and $F_{j}: C \rightarrow H$ be $\zeta$-inverse-strongly monotone, $\eta_{i^{-}}$ inverse-strongly monotone, and $\zeta_{j}$-inverse-strongly monotone, respectively, for $i=1,2$ and $j=1,2$. Let $\left\{T_{n}\right\}_{n=1}^{\infty}$ be a sequence of nonexpansive mappings on $H$ and let $\left\{\lambda_{n}\right\}$ be a sequence in $(0, b]$ for some $b \in(0,1)$. Let $S: C \rightarrow C$ be a uniformly continuous asymptotically $k$-strict pseudocontractive mapping in the intermediate sense for some $0 \leq k<1$ with sequence $\left\{\gamma_{n}\right\} \subset[0, \infty)$ such that $\lim _{n \rightarrow \infty} \gamma_{n}=0$ and $\left\{c_{n}\right\} \subset[0, \infty)$ such that $\lim _{n \rightarrow \infty} c_{n}=0$. Let $V$ be a $\bar{\gamma}$-strongly positive bounded linear operator with $\bar{\gamma}>1$. Let $F: H \rightarrow H$ be a $\kappa$ Lipschitzian and $\eta$-strongly monotone operator with positive constants $\kappa, \eta>0$. Let $Q: H \rightarrow H$ be an l-Lipschitzian mapping with constant $l \geq 0$. Let $0<\mu<2 \eta / \kappa^{2}$ and $0 \leq \gamma l \leq \tau$, where $\tau=1-\sqrt{1-\mu\left(2 \eta-\mu \kappa^{2}\right)}$. Assume that $\Omega:=\cap_{n=1}^{\infty} F i x\left(T_{n}\right) \cap G M E P(\Theta, \varphi, B) \cap V I\left(C, A_{1}\right) \cap V I\left(C, A_{2}\right) \cap$ $\operatorname{GSVI}(G) \cap$ Fix $(S)$ is nonempty and bounded and that either (B1) or (B2) holds. Let $0<\alpha \leq \alpha_{n} \leq 1, k \leq \delta_{n} \leq d<1$ for all $n \geq 1$, and let $\left\{\beta_{n}\right\},\left\{\epsilon_{n}\right\}$, and $\left\{\sigma_{n}\right\}$ be sequences in $(0,1]$. Pick any $x_{0} \in H$ and set $C_{1}=C, x_{1}=P_{C_{1}} x_{0}$. Let $\left\{x_{n}\right\}$ be a sequence generated by the following algorithm:

$$
\begin{gathered}
\Theta\left(u_{n}, y\right)+\varphi(y)-\varphi\left(u_{n}\right)+\left\langle B x_{n}, y-u_{n}\right\rangle \\
+\frac{1}{r_{n}}\left\langle u_{n}-x_{n}, y-u_{n}\right\rangle \geq 0, \quad \forall y \in C, \\
v_{n}=P_{C}\left(I-\lambda_{2, n} A_{2}\right) P_{C}\left(I-\lambda_{1, n} B_{1}\right) u_{n}, \\
z_{n}=\beta_{n} x_{n}+\left(\left(1-\beta_{n}\right) I-\epsilon_{n} V\right) W_{n} G v_{n} \\
+\epsilon_{n}\left[W_{n} x_{n}-\sigma_{n}\left(\mu F\left(W_{n} x_{n}\right)-\gamma Q x_{n}\right)\right], \\
k_{n}=\delta_{n} z_{n}+\left(1-\delta_{n}\right) S^{n} z_{n}, \\
y_{n}=\left(1-\alpha_{n}\right) x_{n}+\alpha_{n} k_{n}, \\
C_{n+1}=\left\{z \in C_{n}:\left\|y_{n}-z\right\|^{2} \leq\left\|x_{n}-z\right\|^{2}+\theta_{n}\right\}, \\
x_{n+1}=P_{C_{n+1}} x_{0}, \quad \forall n \geq 1,
\end{gathered}
$$

where $W_{n}$ is the $W$-mapping defined by (34), $\theta_{n}=\left(\epsilon_{n}+\gamma_{n}\right)(1+$ $\left.\gamma_{n}\right) \Delta_{n}+c_{n}$, and $\Delta_{n}=\sup \left\{\left\|x_{n}-p\right\|^{2}+(\|(I-V) p\|+\|(\gamma Q-\right.$ $\left.\mu F) p \|)^{2} /(\bar{\gamma}-1): p \in \Omega\right\}<\infty$. Suppose that the following conditions are satisfied: (i) $\left\{r_{n}\right\} \subset[e, f] \subset(0,2 \zeta),\left\{\lambda_{i, n}\right\} \subset\left[a_{i}, b_{i}\right] \subset\left(0,2 \eta_{i}\right)$, and $v_{j} \in\left(0,2 \zeta_{j}\right)$ for $i=1,2$ and $j=1,2$;

(ii) $\lim _{n \rightarrow \infty} \epsilon_{n}=0$ and $0<\liminf _{n \rightarrow \infty} \beta_{n} \leq$ $\lim \sup _{n \rightarrow \infty} \beta_{n}<1$.

Then one has the following:

(I) $\left\{x_{n}\right\}$ converges strongly to $x^{*}=P_{\Omega} x_{0}$;

(II) $\left\{x_{n}\right\}$ converges strongly to $x^{*}=P_{\Omega} x_{0}$ provided $\| x_{n}-$ $z_{n} \|=o\left(\epsilon_{n}\right)$ and $\lim _{n \rightarrow \infty} \sigma_{n}=0$, which is the unique solution in $\Omega$ to the VIP

$$
\left\langle(I-V) x^{*}, p-x^{*}\right\rangle \leq 0, \quad \forall p \in \Omega .
$$

Equivalently, $x^{*}=P_{\Omega}(2 I-V) x^{*}$.

Corollary 30. Let $C$ be a nonempty closed convex subset of a real Hilbert space $H$. Let $\Theta$ be a bifunction from $C \times C$ to $\mathbf{R}$ satisfying (A1)-(A4) and let $\varphi: C \rightarrow \mathbf{R} \cup\{+\infty\}$ be a proper lower semicontinuous and convex function. Let $B, A$ : $H \rightarrow H$, and $F_{j}: C \rightarrow H$ be $\zeta$-inverse-strongly monotone, $\xi$ inverse-strongly monotone, and $\zeta_{j}$-inverse-strongly monotone, respectively, for $j=1,2$. Let $\left\{T_{n}\right\}_{n=1}^{\infty}$ be a sequence of nonexpansive mappings on $H$ and let $\left\{\lambda_{n}\right\}$ be a sequence in $(0, b]$ for some $b \in(0,1)$. Let $S: C \rightarrow C$ be a uniformly continuous asymptotically $k$-strict pseudocontractive mapping in the intermediate sense for some $0 \leq k<1$ with sequence $\left\{\gamma_{n}\right\} \subset[0, \infty)$ such that $\lim _{n \rightarrow \infty} \gamma_{n}=0$ and $\left\{c_{n}\right\} \subset[0, \infty)$ such that $\lim _{n \rightarrow \infty} c_{n}=0$. Let $V$ be a $\bar{\gamma}$-strongly positive bounded linear operator with $\bar{\gamma}>1$. Let $F: H \rightarrow H$ be a $\kappa-$ Lipschitzian and $\eta$-strongly monotone operator with positive constants $\kappa, \eta>0$. Let $Q: H \rightarrow H$ be an l-Lipschitzian mapping with constant $l \geq 0$. Let $0<\mu<2 \eta / \kappa^{2}$ and $0 \leq \gamma l \leq \tau$, where $\tau=1-\sqrt{1-\mu\left(2 \eta-\mu \kappa^{2}\right)}$. Assume that $\Omega:=$ $\cap_{n=1}^{\infty} F i x\left(T_{n}\right) \cap G M E P(\Theta, \varphi, B) \cap V I(C, A) \cap G S V I(G) \cap F i x(S)$ is nonempty and bounded and that either (B1) or (B2) holds. Let $0<\alpha \leq \alpha_{n} \leq 1, k \leq \delta_{n} \leq d<1$ for all $n \geq 1$, and let $\left\{\beta_{n}\right\},\left\{\epsilon_{n}\right\}$, and $\left\{\sigma_{n}\right\}$ be sequences in $(0,1]$. Pick any $x_{0} \in H$ and set $C_{1}=C, x_{1}=P_{C_{1}} x_{0}$. Let $\left\{x_{n}\right\}$ be a sequence generated by the following algorithm:

$$
\begin{gathered}
\Theta\left(u_{n}, y\right)+\varphi(y)-\varphi\left(u_{n}\right)+\left\langle B x_{n}, y-u_{n}\right\rangle \\
+\frac{1}{r_{n}}\left\langle u_{n}-x_{n}, y-u_{n}\right\rangle \geq 0, \quad \forall y \in C, \\
v_{n}=P_{C}\left(I-\rho_{n} A\right) u_{n}, \\
z_{n}=\beta_{n} x_{n}+\left(\left(1-\beta_{n}\right) I-\epsilon_{n} V\right) W_{n} G v_{n} \\
+\epsilon_{n}\left[W_{n} x_{n}-\sigma_{n}\left(\mu F\left(W_{n} x_{n}\right)-\gamma Q x_{n}\right)\right], \\
k_{n}=\delta_{n} z_{n}+\left(1-\delta_{n}\right) S^{n} z_{n}, \\
y_{n}=\left(1-\alpha_{n}\right) x_{n}+\alpha_{n} k_{n}, \\
C_{n+1}=\left\{z \in C_{n}:\left\|y_{n}-z\right\|^{2} \leq\left\|x_{n}-z\right\|^{2}+\theta_{n}\right\}, \\
x_{n+1}=P_{C_{n+1}} x_{0}, \quad \forall n \geq 1,
\end{gathered}
$$


where $W_{n}$ is the W-mapping defined by (34), $\theta_{n}=\left(\epsilon_{n}+\gamma_{n}\right)(1+$ $\left.\gamma_{n}\right) \Delta_{n}+c_{n}$, and $\Delta_{n}=\sup \left\{\left\|x_{n}-p\right\|^{2}+(\|(I-V) p\|+\|(\gamma Q-\right.$ $\left.\mu F) p \|)^{2} /(\bar{\gamma}-1): p \in \Omega\right\}<\infty$. Suppose that the following conditions are satisfied:

(i) $\left\{r_{n}\right\} \subset[e, f] \subset(0,2 \zeta),\left\{\rho_{n}\right\} \subset[a, c] \subset(0,2 \xi)$, and $v_{j} \in\left(0,2 \zeta_{j}\right)$ for $j=1,2$;

(ii) $\lim _{n \rightarrow \infty} \epsilon_{n}=0$ and $0<\liminf _{n \rightarrow \infty} \beta_{n} \leq$ $\lim \sup _{n \rightarrow \infty} \beta_{n}<1$.

Then one has the following:

(I) $\left\{x_{n}\right\}$ converges strongly to $x^{*}=P_{\Omega} x_{0}$;

(II) $\left\{x_{n}\right\}$ converges strongly to $x^{*}=P_{\Omega} x_{0}$ provided $\| x_{n}-$ $z_{n} \|=o\left(\epsilon_{n}\right)$ and $\lim _{n \rightarrow \infty} \sigma_{n}=0$, which is the unique solution in $\Omega$ to the VIP

$$
\left\langle(I-V) x^{*}, p-x^{*}\right\rangle \leq 0, \quad \forall p \in \Omega \text {. }
$$

Equivalently, $x^{*}=P_{\Omega}(2 I-V) x^{*}$.

Corollary 31. Let $C$ be a nonempty closed convex subset of a real Hilbert space $H$. Let $\Theta$ be a bifunction from $C \times C$ to $\mathbf{R}$ satisfying (A1)-(A4) and let $\varphi: C \rightarrow \mathbf{R} \cup\{+\infty\}$ be a proper lower semicontinuous and convex function. Let $B, A$ : $H \rightarrow H$, and $F_{j}: C \rightarrow H$ be $\zeta$-inverse-strongly monotone, $\xi$ inverse-strongly monotone, and $\zeta_{j}$-inverse-strongly monotone, respectively, for $j=1,2$. Let $\left\{T_{n}\right\}_{n=1}^{\infty}$ be a sequence of nonexpansive mappings on $H$ and let $\left\{\lambda_{n}\right\}$ be a sequence in $(0, b]$ for some $b \in(0,1)$. Let $S: C \rightarrow C$ be a uniformly continuous asymptotically $k$-strict pseudocontractive mapping for some $0 \leq k<1$ with sequence $\left\{\gamma_{n}\right\} \subset[0, \infty)$ such that $\lim _{n \rightarrow \infty} \gamma_{n}=0$. Let $V$ be a $\bar{\gamma}$-strongly positive bounded linear operator with $\bar{\gamma}>1$. Let $F: H \rightarrow H$ be $a \kappa-$ Lipschitzian and $\eta$-strongly monotone operator with positive constants $\kappa, \eta>0$. Let $Q: H \rightarrow H$ be an l-Lipschitzian mapping with constant $l \geq 0$. Let $0<\mu<2 \eta / \kappa^{2}$ and $0 \leq \gamma l \leq \tau$, where $\tau=1-\sqrt{1-\mu\left(2 \eta-\mu \kappa^{2}\right)}$. Assume that $\Omega:=$ $\cap_{n=1}^{\infty} \operatorname{Fix}\left(T_{n}\right) \cap \operatorname{GMEP}(\Theta, \varphi, B) \cap \operatorname{VI}(C, A) \cap \operatorname{GSVI}(G) \cap F i x(S)$ is nonempty and bounded and that either (B1) or (B2) holds. Let $0<\alpha \leq \alpha_{n} \leq 1, k \leq \delta_{n} \leq d<1$ for all $n \geq 1$, and let $\left\{\beta_{n}\right\},\left\{\epsilon_{n}\right\}$, and $\left\{\sigma_{n}\right\}$ be sequences in $(0,1]$. Pick any $x_{0} \in H$ and set $C_{1}=C, x_{1}=P_{C_{1}} x_{0}$. Let $\left\{x_{n}\right\}$ be a sequence generated by the following algorithm:

$$
\begin{gathered}
\Theta\left(u_{n}, y\right)+\varphi(y)-\varphi\left(u_{n}\right)+\left\langle B x_{n}, y-u_{n}\right\rangle \\
+\frac{1}{r_{n}}\left\langle u_{n}-x_{n}, y-u_{n}\right\rangle \geq 0, \quad \forall y \in C, \\
v_{n}=P_{C}\left(I-\rho_{n} A\right) u_{n},
\end{gathered}
$$

$$
\begin{gathered}
z_{n}=\beta_{n} x_{n}+\left(\left(1-\beta_{n}\right) I-\epsilon_{n} V\right) W_{n} G v_{n} \\
+\epsilon_{n}\left[W_{n} x_{n}-\sigma_{n}\left(\mu F\left(W_{n} x_{n}\right)-\gamma Q x_{n}\right)\right], \\
k_{n}=\delta_{n} z_{n}+\left(1-\delta_{n}\right) S^{n} z_{n}, \\
y_{n}=\left(1-\alpha_{n}\right) x_{n}+\alpha_{n} k_{n}, \\
C_{n+1}=\left\{z \in C_{n}:\left\|y_{n}-z\right\|^{2} \leq\left\|x_{n}-z\right\|^{2}+\theta_{n}\right\}, \\
x_{n+1}=P_{C_{n+1}} x_{0}, \quad \forall n \geq 1,
\end{gathered}
$$

where $W_{n}$ is the $W$-mapping defined by (34), $\theta_{n}=\left(\epsilon_{n}+\gamma_{n}\right)(1+$ $\left.\gamma_{n}\right) \Delta_{n}$, and $\Delta_{n}=\sup \left\{\left\|x_{n}-p\right\|^{2}+(\|(I-V) p\|+\|(\gamma Q-\right.$ $\left.\mu F) p \|)^{2} /(\bar{\gamma}-1): p \in \Omega\right\}<\infty$. Suppose that the following conditions are satisfied:

(i) $\left\{r_{n}\right\} \subset[e, f] \subset(0,2 \zeta),\left\{\rho_{n}\right\} \subset[a, c] \subset(0,2 \xi)$, and $v_{j} \in\left(0,2 \zeta_{j}\right)$ for $j=1,2$;

(ii) $\lim _{n \rightarrow \infty} \epsilon_{n}=0$ and $0<\liminf _{n \rightarrow \infty} \beta_{n} \leq$ $\limsup _{n \rightarrow \infty} \beta_{n}<1$.

Then one has the following:

(I) $\left\{x_{n}\right\}$ converges strongly to $x^{*}=P_{\Omega} x_{0}$;

(II) $\left\{x_{n}\right\}$ converges strongly to $x^{*}=P_{\Omega} x_{0}$ provided $\| x_{n}-$ $z_{n} \|=o\left(\epsilon_{n}\right)$ and $\lim _{n \rightarrow \infty} \sigma_{n}=0$, which is the unique solution in $\Omega$ to the VIP

$$
\left\langle(I-V) x^{*}, p-x^{*}\right\rangle \leq 0, \quad \forall p \in \Omega \text {. }
$$

Equivalently, $x^{*}=P_{\Omega}(2 I-V) x^{*}$.

Remark 32. Let $A: C \rightarrow H$ be $\xi$-inverse-strongly monotone and let $F_{i}: C \rightarrow H$ be $v_{j}$-inverse-strongly monotone for $j=1,2$. Let $Q: C \rightarrow C$ be a $\rho$-contraction with $\rho \in[0,1)$, and let $S: C \rightarrow C$ be a uniformly continuous asymptotically $k$-strict pseudocontractive mapping in the intermediate sense for some $0 \leq k<1$ with sequence $\left\{\gamma_{n}\right\} \subset[0, \infty)$ such that $\lim _{n \rightarrow \infty} \gamma_{n}=0$ and $\left\{c_{n}\right\} \subset[0, \infty)$ such that $\lim _{n \rightarrow \infty} c_{n}=0$. Assume that $\Omega:=\operatorname{VI}(C, A) \cap \operatorname{GSVI}(G) \cap \operatorname{Fix}(S)$ is nonempty and bounded. In [11], Guu et al. introduced and analyzed a hybrid viscosity CQ iterative algorithm for finding a point $p \epsilon$ $\Omega$ :

$$
\begin{aligned}
x_{1} & =x \in C \quad \text { choosen arbitrarily, } \\
y_{n} & =P_{C}\left(x_{n}-\rho_{n} A x_{n}\right), \\
t_{n} & =\alpha_{n} Q x_{n}+\left(1-\alpha_{n}\right) G y_{n}, \\
z_{n} & =\left(1-\mu_{n}-v_{n}\right) x_{n}+\mu_{n} t_{n}+\nu_{n} S^{n} t_{n}, \\
C_{n} & =\left\{z \in C:\left\|z_{n}-z\right\|^{2} \leq\left\|x_{n}-z\right\|^{2}+\theta_{n}\right\}, \\
Q_{n} & =\left\{z \in C:\left\langle x_{n}-z, x-x_{n}\right\rangle \geq 0\right\}, \\
x_{n+1} & =P_{C_{n} \cap Q_{n}} x, \quad \forall n \geq 1,
\end{aligned}
$$


where $v_{j} \in\left(0,2 \zeta_{j}\right)$ for $j=1,2, \theta_{n}=\left(\alpha_{n}+\gamma_{n}\right) \Delta_{n}+c_{n}$; $\Delta_{n}=\sup \left\{\left\|x_{n}-p\right\|^{2}+\left(1+\gamma_{n}\right) /(1-\rho)\|(I-Q) p\|^{2}: p \in \Omega\right\}<$ $\infty ;\left\{\rho_{n}\right\}$ is a sequence in $(0,2 \xi)$; and $\left\{\alpha_{n}\right\},\left\{\mu_{n}\right\}$, and $\left\{\nu_{n}\right\}$ are three sequences in $[0,1]$ such that $\mu_{n}+\nu_{n} \leq 1$ for all $n \geq 1$. The authors of [11] proved that under suitable conditions $\left\{x_{n}\right\}$ converges strongly to $P_{\Omega} x$; see [11, Theorem 3.1] for more details.

Theorem 28 extends, improves, supplements, and develops [11, Theorem 3.1] in the following aspects.

(i) The problem of finding a point $p \in \cap_{n=1}^{\infty} \operatorname{Fix}\left(T_{n}\right) \cap$ $\cap_{k=1}^{M} \operatorname{GMEP}\left(\Theta_{k}, \varphi_{k}, B_{k}\right) \cap \cap_{i=1}^{N} \operatorname{VI}\left(C, A_{i}\right) \cap \operatorname{GSVI}(G) \cap$ Fix $(S)$ in Theorem 28 is very different from the problem of finding a point $p \in \operatorname{VI}(C, A) \cap \operatorname{GSVI}(G) \cap$ Fix $(S)$ in [11, Theorem 3.1]. There is no doubt that our problem of finding a point $p \in \cap_{n=1}^{\infty} F i x\left(T_{n}\right) \cap$ $\cap_{k=1}^{M} \operatorname{GMEP}\left(\Theta_{k}, \varphi_{k}, B_{k}\right) \cap \cap_{i=1}^{N} \operatorname{VI}\left(C, A_{i}\right) \cap \operatorname{GSVI}(G) \cap$ Fix $(S)$ is more general and more subtle than the problem of finding a point $p \in \operatorname{VI}(C, A) \cap \operatorname{GSVI}(G) \cap$ Fix $(S)$ in [11, Theorem 3.1].

(ii) The iterative scheme in [11, Theorem 3.1] is extended to develop the iterative scheme in Theorem 28 by virtue of Cai and $\mathrm{Bu}$ iterative algorithm in [21, Theorem 3.1] and Ceng et al. iterative one in [8, Theorem 3.1]. The iterative scheme in Theorem 28 is more advantageous and more flexible than the iterative scheme in [11, Theorem 3.1] because it involves solving four problems: the GSVI (8), finitely many GMEPs, finitely many VIPs, and the common fixed point problem of an asymptotically strict pseudocontractive mapping in the intermediate sense and infinitely many nonexpansive mappings on $H$.

(iii) The iterative scheme in Theorem 28 is very different from the iterative scheme in [11, Theorem 3.1] because the iterative scheme in our theorem (Theorem 28) involves hybrid steepest-descent method in [7], strongly positive bounded linear operator technique, finitely many GMEPs, finitely many VIPs, and infinitely many nonexpansive mappings. The proof in [11, Theorem 3.1] makes use of Proposition CWY and the properties of asymptotically strict pseudocontractive mapping in the intermediate sense (see Lemmas 17-20). However, the proof of Theorem 28 depends on not only Proposition CWY and Lemmas 17-20 but also Proposition 8 , the properties of strongly positive bounded linear operator $V$, and the ones of the $W$ mapping $W_{n}$ and $T^{\lambda}$-mapping (see Lemmas 12,13 , and 15) because there are the mapping $T_{r_{k, n}}^{\left(\Theta_{k}, \varphi_{k}\right)}$, infinitely many nonexpansive mappings $\left\{T_{n}\right\}_{n=1}^{\infty}, \kappa$-Lipschitzian and $\eta$-strongly monotone operator $F$, and strongly positive bounded linear operator $V$ appearing in the iterative scheme of our theorem (Theorem 28).

(iv) The proof of Theorem 28 combines $\mathrm{Cai}$ and $\mathrm{Bu}$ convergence analysis for their iterative algorithm to solve finitely many GMEPs, finitely many VIPs, and the fixed point problem of an asymptotically strict pseudocontractive mapping in the intermediate sense (see [21, Theorem 3.1]); the convergence analysis for the $W$-mapping approach to fixed points of infinitely many nonexpansive mappings and strongly positive bounded linear operator technique; and Ceng, Guu, and Yao convergence analysis for hybrid iterative method (see [11, Theorem 3.1]).

Remark 33. Theorem 28 also extends, improves, supplements, and develops Ceng et al. [8, Theorem 3.1] in the following aspects.

(i) The problem of finding a point $p \in \cap_{n=1}^{\infty} \operatorname{Fix}\left(T_{n}\right) \cap$ $\cap_{k=1}^{M} \operatorname{GMEP}\left(\Theta_{k}, \varphi_{k}, B_{k}\right) \cap \cap_{i=1}^{N} \operatorname{VI}\left(C, A_{i}\right) \cap \operatorname{GSVI}(G) \cap$ Fix $(S)$ in Theorem 28 is very different from the problem of finding a point $p \in \cap_{i=1}^{N} F i x\left(T_{i}\right) \cap$ $\operatorname{GMEP}(\Theta, \varphi, A)$ in Ceng et al. [8, Theorem 3.1]. Here our problem of finding a point $p \in \cap_{n=1}^{\infty} \operatorname{Fix}\left(T_{n}\right) \cap$ $\cap_{k=1}^{M} \operatorname{GMEP}\left(\Theta_{k}, \varphi_{k}, B_{k}\right) \cap \cap_{i=1}^{N} \operatorname{VI}\left(C, A_{i}\right) \cap \operatorname{GSVI}(G) \cap$ Fix $(S)$ is put forth after one GMEP; finitely many nonexpansive mappings in their problem are replaced by finitely many GMEPs and infinitely many nonexpansive mappings, respectively; and the GSVI (8), finitely many VIPs, and the fixed point problem of an asymptotically strict pseudocontractive mapping in the intermediate sense are added to their problem.

(ii) The iterative scheme in [8, Theorem 3.1] is extended to develop the iterative scheme in our theorem (Theorem 28) by virtue of Korpelevich's extragradient method [22], shrinking projection method, Mann iterative method, and strongly positive bounded linear operator technique. The iterative scheme in Theorem 28 is put forth after $u_{n}=T_{r_{n}}^{(\Theta, \varphi)}\left(I-r_{n} A\right) x_{n}$ and $W_{n} u_{n}$ in [8, Theorem 3.1] are replaced by $u_{n}=$ $\Delta_{n}^{M} x_{n}$ and $W_{n} G \Lambda_{n}^{N} u_{n}$, respectively.

(iii) The iterative scheme in Theorem 28 is very different from the iterative scheme in [8, Theorem 3.1] because the iterative scheme in Theorem 28 involves Korpelevich's extragradient method [22], shrinking projection method, Mann iterative method, and strongly positive bounded linear operator technique. The proof of [8, Theorem 3.1] makes use of Proposition 8. However, the proof of Theorem 28 depends on not only Proposition 8 but also Proposition CWY, the properties of strongly positive bounded linear operator, and the ones of asymptotically strict pseudocontractive mapping in the intermediate sense (see Lemmas 17-20) because there are the SGEP (8), finitely many GMEPs, asymptotically strict pseudocontractive mapping $S$ in the intermediate sense, and the strongly positive bounded linear operator $V$ appearing in the iterative scheme of our theorem (Theorem 28).

(iv) The proof of Theorem 28 involves the convergence analysis for Korpelevich's extragradient method to solve the SGEP (8), finitely many GMEPs, and finitely many VIPs; the convergence analysis for the $W$-mapping approach to fixed points of infinitely many nonexpansive mappings and strongly positive 
bounded linear operator technique; and Ceng, Guu, and Yao convergence analysis for viscosity approximation method and hybrid steepest-descent method (see [23, Theorem 4.2] and [8, Theorem 3.1]).

\section{Conflict of Interests}

The authors declare that there is no conflict of interests regarding the publication of this paper.

\section{Acknowledgments}

This research was partially supported by the National Science Foundation of China (11071169), the Innovation Program of Shanghai Municipal Education Commission (09ZZ133), and the Ph.D. Program Foundation of Ministry of Education of China (20123127110002). This work was supported partly by the National Science Council of the Republic of China. This research was partially supported by a grant from NSC.

\section{References}

[1] J.-W. Peng and J.-C. Yao, "A new hybrid-extragradient method for generalized mixed equilibrium problems, fixed point problems and variational inequality problems," Taiwanese Journal of Mathematics, vol. 12, no. 6, pp. 1401-1432, 2008.

[2] V. Colao, G. Marino, and H.-K. Xu, "An iterative method for finding common solutions of equilibrium and fixed point problems," Journal of Mathematical Analysis and Applications, vol. 344, no. 1, pp. 340-352, 2008.

[3] L.-C. Ceng and J.-C. Yao, "A hybrid iterative scheme for mixed equilibrium problems and fixed point problems," Journal of Computational and Applied Mathematics, vol. 214, no. 1, pp. 186201, 2008.

[4] S. Takahashi and W. Takahashi, "Strong convergence theorem for a generalized equilibrium problem and a nonexpansive mapping in a Hilbert space," Nonlinear Analysis: Theory, Methods \& Applications, vol. 69, no. 3, pp. 1025-1033, 2008.

[5] L. C. Ceng, A. Petruşel, and J. C. Yao, "Iterative approaches to solving equilibrium problems and fixed point problems of infinitely many nonexpansive mappings," Journal of Optimization Theory and Applications, vol. 143, no. 1, pp. 37-58, 2009.

[6] L.-C. Ceng, C.-y. Wang, and J.-C. Yao, "Strong convergence theorems by a relaxed extragradient method for a general system of variational inequalities," Mathematical Methods of Operations Research, vol. 67, no. 3, pp. 375-390, 2008.

[7] I. Yamada, "The hybrid steepest descent method for the variational inequality problem over the intersection of fixed point sets of nonexpansive mappings," in Inherently Parallel Algorithms in Feasibility and Optimization and Their Applications (Haifa, 2000), vol. 8 of Studies in Computational Mathematics, pp. 473-504, North-Holland, Amsterdam, The Netherlands, 2001.

[8] L.-C. Ceng, S.-M. Guu, and J.-C. Yao, "Hybrid iterative method for finding common solutions of generalized mixed equilibrium and fixed point problems," Fixed Point Theory and Applications, vol. 2012, article 92, 2012.

[9] T.-H. Kim and H.-K. Xu, "Convergence of the modified Mann's iteration method for asymptotically strict pseudo-contractions," Nonlinear Analysis: Theory, Methods \& Applications, vol. 68, no. 9, pp. 2828-2836, 2008.
[10] D. R. Sahu, H.-K. Xu, and J.-C. Yao, "Asymptotically strict pseudocontractive mappings in the intermediate sense," Nonlinear Analysis: Theory, Methods \& Applications, vol. 70, no. 10, pp. 3502-3511, 2009.

[11] S.-M. Guu, L.-C. Ceng, and J.-C. Yao, "Finding common solutions of a variational inequality, a general system of variational inequalities, and a fixed-point problem via a hybrid extragradient method," Fixed Point Theory and Applications, vol. 2011, Article ID 626159, 2011.

[12] L.-C. Ceng and J.-C. Yao, "Strong convergence theorems for variational inequalities and fixed point problems of asymptotically strict pseudocontractive mappings in the intermediate sense," Acta Applicandae Mathematicae, vol. 115, no. 2, pp. 167191, 2011.

[13] S. Huang, "Hybrid extragradient methods for asymptotically strict pseudo-contractions in the intermediate sense and variational inequality problems," Optimization, vol. 60, no. 6, pp. 739-754, 2011.

[14] H.-K. Xu, "Averaged mappings and the gradient-projection algorithm," Journal of Optimization Theory and Applications, vol. 150, no. 2, pp. 360-378, 2011.

[15] C. Byrne, "A unified treatment of some iterative algorithms in signal processing and image reconstruction," Inverse Problems, vol. 20, no. 1, pp. 103-120, 2004.

[16] K. Geobel and W. A. Kirk, Topics on Metric Fixed-Point Theory, Cambridge University Press, Cambridge, UK, 1990.

[17] J. G. O’Hara, P. Pillay, and H.-K. Xu, "Iterative approaches to convex feasibility problems in Banach spaces," Nonlinear Analysis: Theory, Methods \& Applications, vol. 64, no. 9, pp. 2022-2042, 2006.

[18] H. K. Xu and T. H. Kim, "Convergence of hybrid steepestdescent methods for variational inequalities," Journal of Optimization Theory and Applications, vol. 119, no. 1, pp. 185-201, 2003.

[19] C. Martinez-Yanes and H.-K. Xu, "Strong convergence of the CQ method for fixed point iteration processes," Nonlinear Analysis: Theory, Methods \& Applications, vol. 64, no. 11, pp. 2400-2411, 2006.

[20] Y. Yao, Y.-C. Liou, and J.-C. Yao, "Convergence theorem for equilibrium problems and fixed point problems of infinite family of nonexpansive mappings," Fixed Point Theory and Applications, vol. 2007, article 12, Article ID 64363, 2007.

[21] G. Cai and S. Bu, "Strong and weak convergence theorems for general mixed equilibrium problems and variational inequality problems and fixed point problems in Hilbert spaces," Journal of Computational and Applied Mathematics, vol. 247, pp. 34-52, 2013.

[22] G. M. Korpelevič, "An extragradient method for finding saddle points and for other problems," Ėkonomika i Matematicheskie Metody, vol. 12, no. 4, pp. 747-756, 1976.

[23] L.-C. Ceng, Q. H. Ansari, and J.-C. Yao, "Some iterative methods for finding fixed points and for solving constrained convex minimization problems," Nonlinear Analysis: Theory, Methods \& Applications, vol. 74, no. 16, pp. 5286-5302, 2011. 


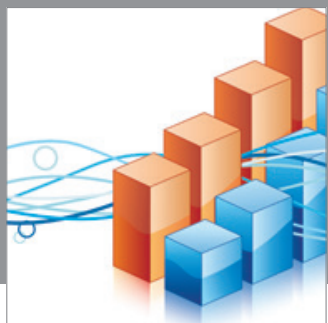

Advances in

Operations Research

mansans

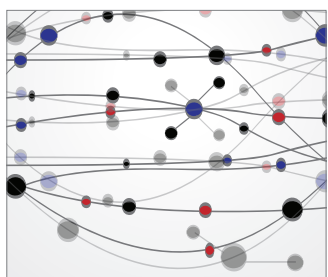

The Scientific World Journal
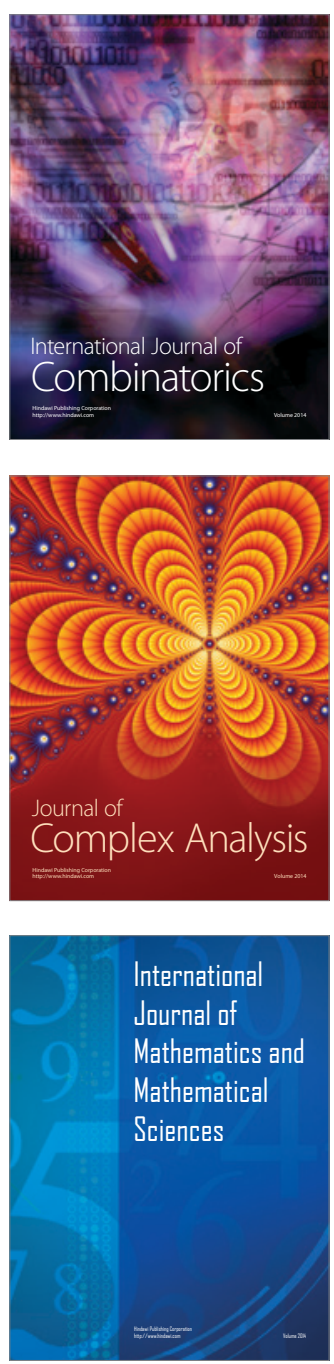
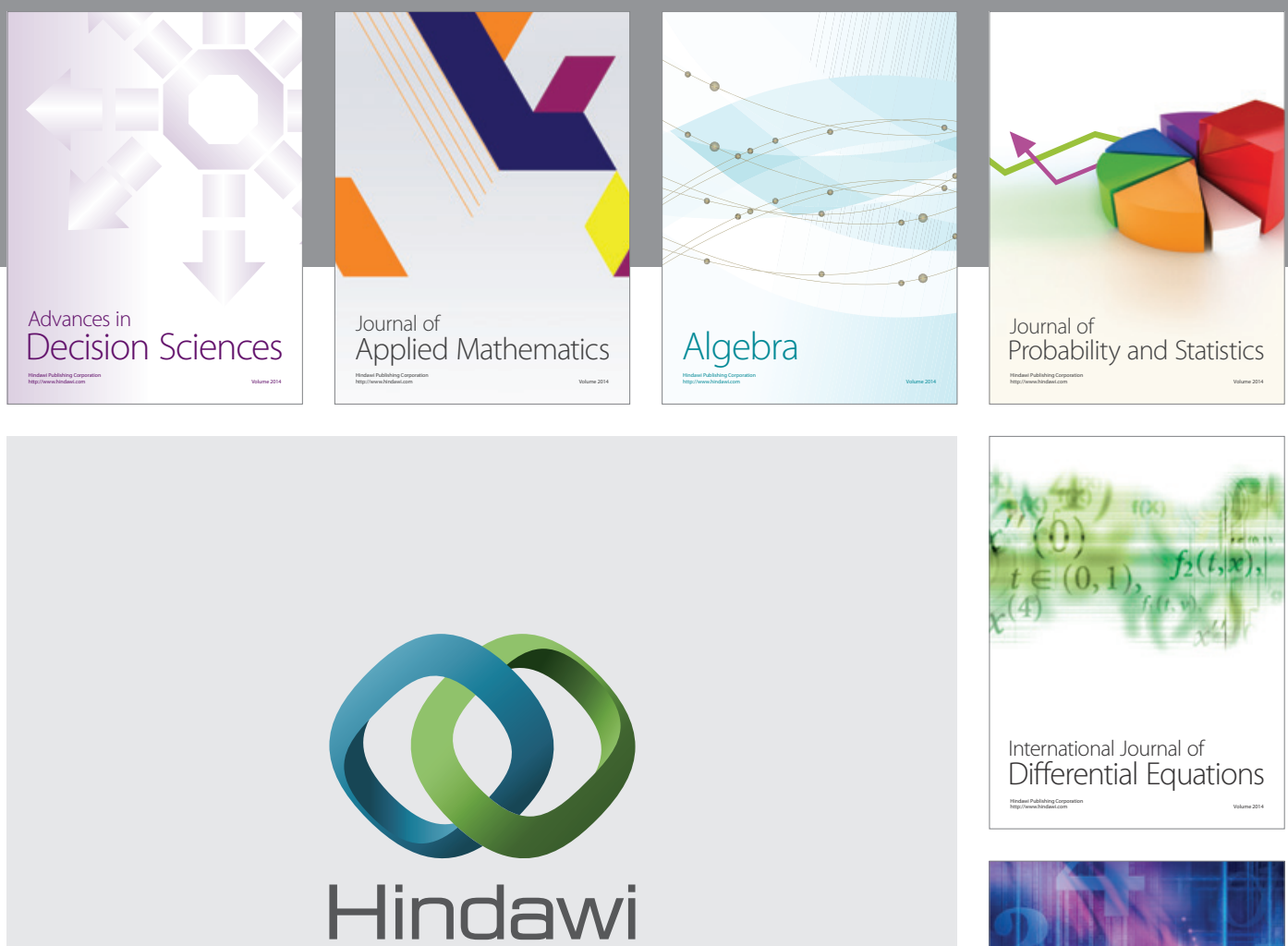

Submit your manuscripts at http://www.hindawi.com
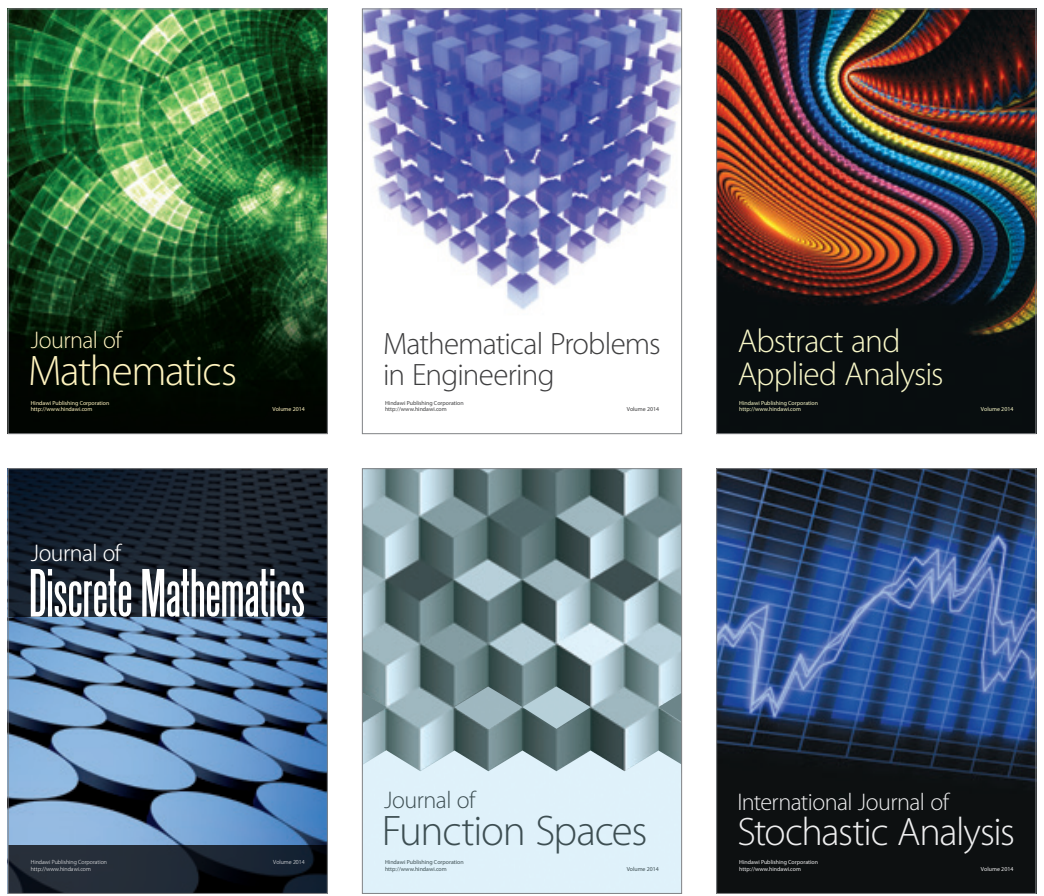

Journal of

Function Spaces

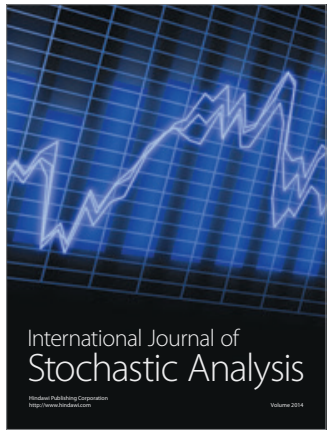

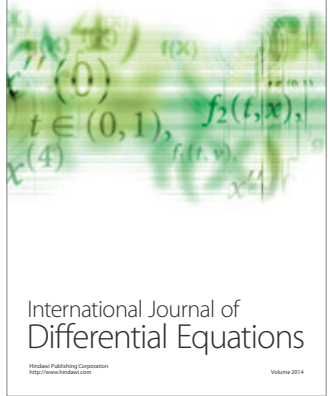
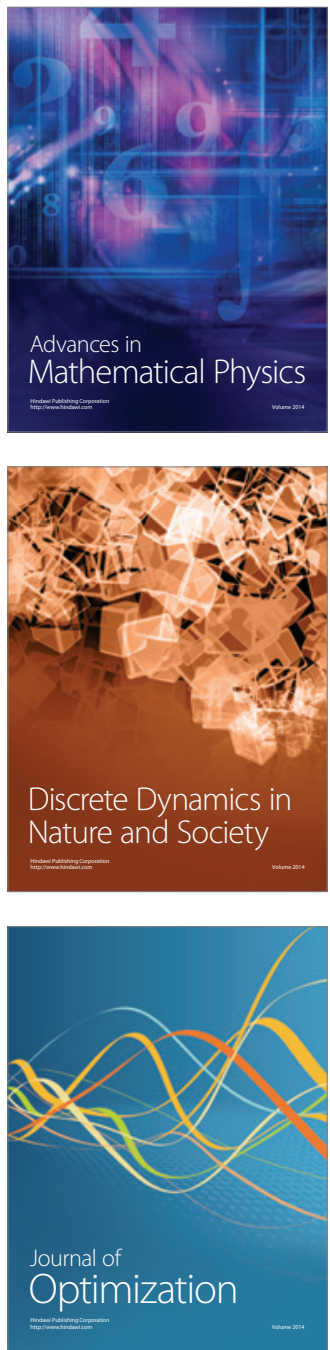